University of Louisville

ThinkIR: The University of Louisville's Institutional Repository

Electronic Theses and Dissertations

$5-2011$

\title{
A multi-objective decision support system for worker-task assignments and workforce training.
}

Brandon B. Elmes

University of Louisville

Follow this and additional works at: https://ir.library.louisville.edu/etd

\section{Recommended Citation}

Elmes, Brandon B., "A multi-objective decision support system for worker-task assignments and workforce training." (2011). Electronic Theses and Dissertations. Paper 401.

https://doi.org/10.18297/etd/401

This Master's Thesis is brought to you for free and open access by ThinkIR: The University of Louisville's Institutional Repository. It has been accepted for inclusion in Electronic Theses and Dissertations by an authorized administrator of ThinkIR: The University of Louisville's Institutional Repository. This title appears here courtesy of the author, who has retained all other copyrights. For more information, please contact thinkir@louisville.edu. 


\title{
A MULTI-OBJECTIVE DECISION SUPPORT SYSTEM FOR WORKER-TASK
} ASSIGNMENTS AND WORKFORCE TRAINING

\author{
By \\ Brandon B. Elmes \\ B.S., University of Louisville, 2007
}

\begin{abstract}
A Thesis
Submitted to the Faculty of the University of Louisville J.B. Speed School of Engineering as Partial Fulfillment of the Requirements for the Professional Degree

MASTER OF ENGINEERING

Department of Industrial Engineering
\end{abstract}

May 2011 



\section{A MULTI-OBJECTIVE DECISION SUPPORT SYSTEM FOR WORKER-TASK ASSIGNMENTS AND WORKFORCE TRAINING}

Submitted by:

Brandon B. Elmes

A Thesis Approved on

(Date)

by the Following Reading and Examination Committee:

Gerald W. Evans, Thesis Director

Gail W. DePuy, Thesis Director

Rammohan K. Ragade, Committee Member 


\section{ACKNOWLEDGEMENTS}

The author received considerable help from both Thesis Directors: Dr. Gerald Evans and

Dr. Gail DePuy. Thank you for your time, effort, and patience. Also, thanks to Dr. Rammoham Ragade for his time as a thesis committee member.

The author acknowledges the assistance of the NSWC in Crane, IN which provided data for the mathematical model. 


\begin{abstract}
This paper models a realistic problem involving workforce assignment and training for a large manufacturing environment. In this particular environment, the workforce is undertrained and most assignments will result in necessary training. This problem was previously addressed as a single objective problem. This paper expands to a multi-objective formulation. This is a more accurate reflection of the problem because almost all real world problems have many objectives which can be conflicting.
\end{abstract}

The program developed in this paper is designed for use by supervisors in the production setting. A two stage program is designed where the first stage generates initial solutions by solving each objective function idependently of the others. MetaRaPS — a modified greedy algorithm—is used to find these solutions. The user selects one of these solutions to carry into the second stage: compromise programming. The second stage uses input from the user in an iterative and intuitive fashion. This input guides the program to the solution which the user determines is the best compromise solution.

Meta-RaPS is effective at finding a good solution extremely quickly. There is an important trade-off between the quality of solutions and computational run-time which will need to be tweaked for a specific application. The compromise programming stage could benefit from coding improvements; however, it is still effective at allowing the user to guide the program towards the best compromise solution by assigning trade-off values between objectives 


\section{TABLE OF CONTENTS}

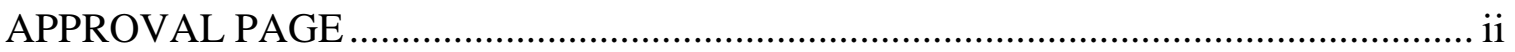

ACKNOWLEDGEMENTS ..............................................................................

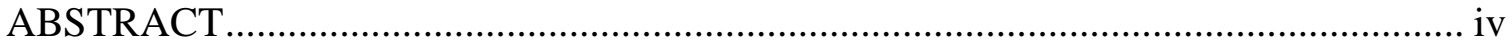

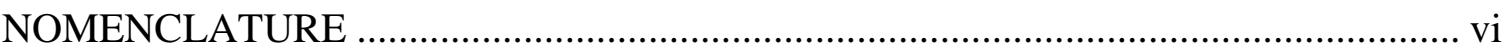

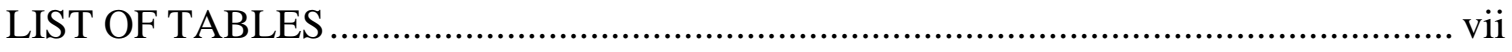

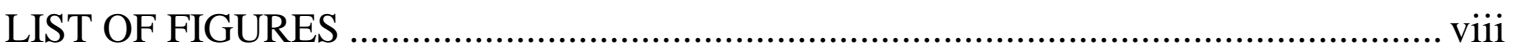

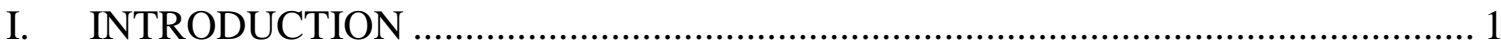

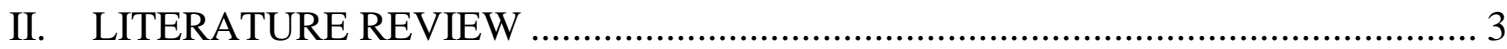

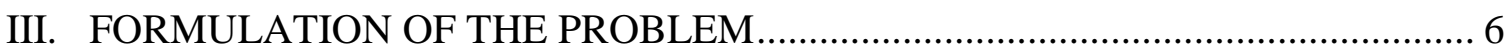

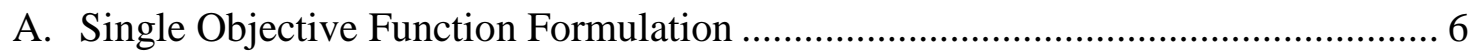

B. Extension to Multi-Objective Formulation ........................................................... 8

1. Maximize Total Training Received .......................................................... 9

2. Maximize Worker Satisfaction ............................................................... 9

3. Goal of the Multi-Objective Decision Support System..................................... 11

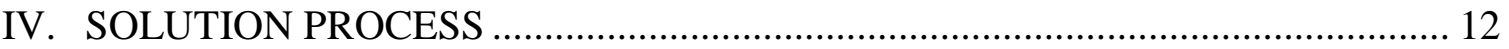

A. Multiple Stage Decision Support System....................................................... 13

V. PSEUDO-CODE AND INPUT FROM THE USER .............................................. 16

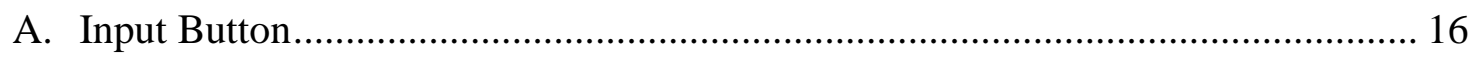

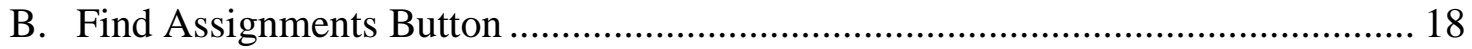

C. Pseudo-code for Decision Support System (DSS) ……......................................... 22

1. Key Variables used in Pseudo-code ........................................................... 22

2. Generalized Meta-RaPS Pseudo-code ......................................................... 23

3. InitialTrainingCostSoln Pseudo-code …………......................................... 23

4. InitialSkillGapSoln Pseudo-code............................................................... 26

5. InitialWorkerPrefSoln Pseudo-code ........................................................... 27

6. Compromise Programming Pseudo-code ………………............................... 30

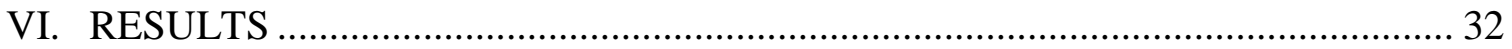

A. Minimize Training Cost Results.................................................................... 33

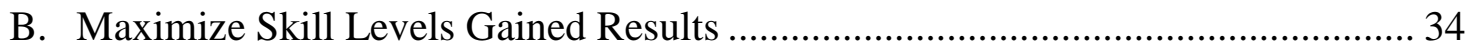

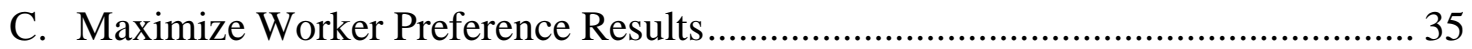

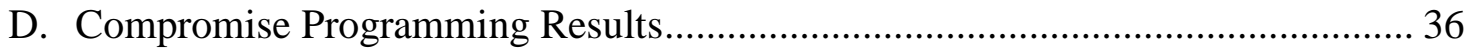

VII. CONCLUSIONS AND RECOMMENDATIONS ………………………….......... 37 
APPENDIX A-Results for Minimize Total Training Cost .............................. 43

APPENDIX B-Results for Maximize Skill Levels Gained.............................. 46

APPENDIX C - Results for Maximize Worker Preference ................................ 49

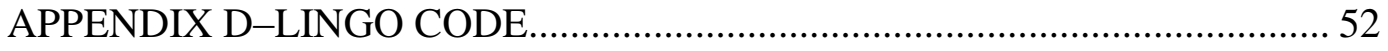

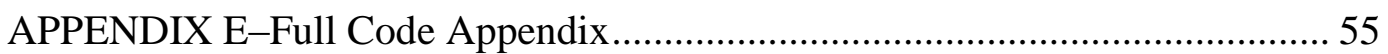


NOMENCLATURE:

$\{\mathrm{j}\}=$ set of skills needed to perform task $\mathrm{j}$

$\mathrm{S}_{\mathrm{ik}}=$ worker i's skill level for skill $\mathrm{k}$

$\mathrm{R}_{\mathrm{jk}}=$ required skill level for task j's skill $\mathrm{k}$

$\mathrm{T}_{\mathrm{j}}=$ length $(\mathrm{hr})$ of task $\mathrm{j}$

$\mathrm{A}_{\mathrm{i}}=$ capacity $(\mathrm{hr})$ of worker $\mathrm{i}$

$\mathrm{C}_{\mathrm{klm}}=$ cost associated with raising a worker's skill level on skill $\mathrm{k}$ from level 1 to level $\mathrm{m}$

$\mathrm{E}_{\mathrm{klm}}=$ time required $(\mathrm{hr})$ to raise a worker's skill level on skill $\mathrm{k}$ from level 1 to level $\mathrm{m}$

$P_{i k}= \begin{cases}1 & \text { workeri would like additional training in skill } \mathrm{k} \\ 0 & \text { otherwise }\end{cases}$

$\mathrm{X}_{\mathrm{ij}}= \begin{cases}1 & \text { workeri assigned to task } \mathrm{j} \\ 0 & \text { otherwise }\end{cases}$

$\mathrm{Z}_{\mathrm{ikS}} \mathrm{s}_{\mathrm{ik}} \mathrm{m}= \begin{cases}1 & \text { workeri receives training on skill } \mathrm{k} \text { to raise skill level from } \mathrm{S}_{\mathrm{ik}} \text { to } \mathrm{m} \\ 0 & \text { otherwise }\end{cases}$

$\mathrm{N}_{\mathrm{ik}}= \begin{cases}1 & \text { workeri does not need further training in skill } \mathrm{k} \\ 0 & \text { otherwise }\end{cases}$ 


\section{LIST OF TABLES}

TABLE I —SAMPLE PROBLEM SIZES .............................................................4

TABLE II— MINIMIZE TRAINING COST RESULTS ...........................................33

TABLE III- MAXIMIZE SKILL LEVELS GAINED RESULTS ..............................34

TABLE IV-MAXIMIZE WORKER PREFERENCE RESULTS ...............................35 


\section{LIST OF FIGURES}

FIGURE 1 - Demonstration of Skill Levels and Skill Gaps .......................................2

FIGURE 2 - Flowchart of the Solution Process ..........................................................15

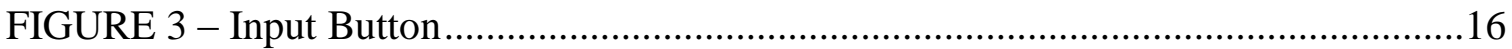

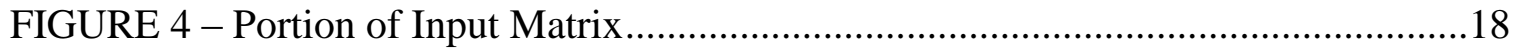

FIGURE 5 - Find Assignments Button .............................................................18

FIGURE 6 - Initial Solution (Stage 1 Complete) .......................................................19

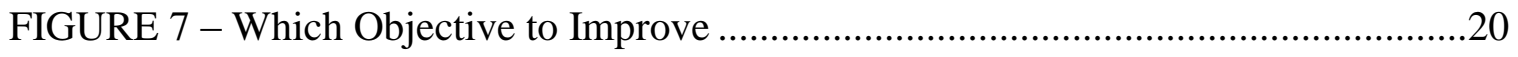

FIGURE 8 - How Much to Sacrifice Each Objective .............................................20

FIGURE 9 - Solution After Compromise Programming ........................................21 


\section{INTRODUCTION}

The Naval Surface Warfare Center (NSWC) has a division located in Crane, Indiana. NSWC Crane is a shore command of the US Navy. This division is a shore command of the United States Navy under the Naval Sea Systems Command headquartered in Washington D.C. NSWC specializes in the acquisition and fleet support of electronics, ordinance products, and electronic warfare products. NSWC routinely bids on a wide variety of remanufacturing work from many different military organizations.

One of the key challenges in preparing these bids is determining how to train their current workforce to keep up with the new technology the military continues to develop. NSWC wants to maintain their current workforce without eliminating any current workers. So, if the current workforce does not have the skill set to complete the necessary tasks, training will be required.

In formulating this problem, there are a series of tasks which will be completed by a worker. Each worker has a particular skill set which they have obtained from previous training. In addition, each worker has a skill level associated with each skill. These levels range from novice to expert (1-5). So, each worker has a particular skill set defined by the skill level. Similarly, each task has a skill set required to complete the task at a desired quality. So, just as each worker has a skill set with associated skill levels, each task has a skill set with associated skill level.

The problem then becomes a task of assigning tasks to workers. It is possible that only one task is assigned to a worker; but it is also possible for more than one task to be assigned to a worker. If a worker is assigned to a task where his skill level is below the 
level required for the task, then a skill gap is present (Figure 1). In this case, the worker will need to be trained to attain the necessary skill level as required by that task. This, of course, incurs a training cost.

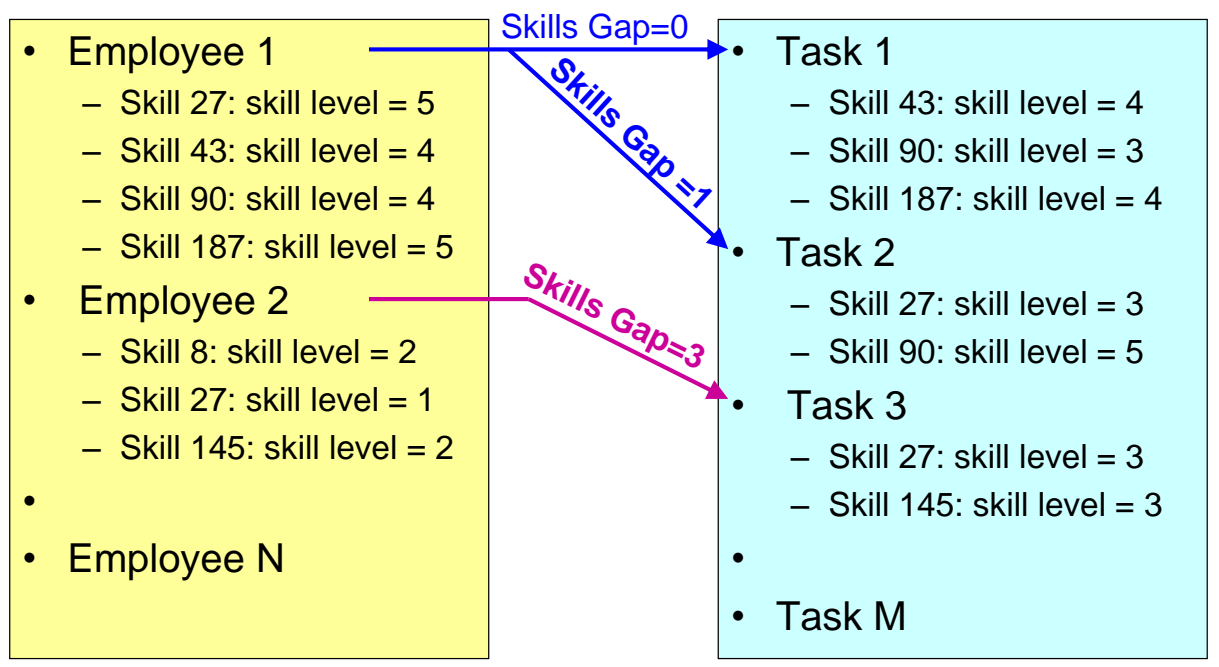

FIGURE 1 - Demonstration of Skill Levels and Skill Gaps

In Figure 1, if Employee 1 is assigned to Task 1, there is no skill gap present. This is because the employee's skill level meets or exceeds the skill level required by the task. On the other hand, if Employee 1 is assigned to Task 2, a skill gap is present. This is because Skill 90 requires a level of 5 whereas the employee only has a skill level of 4 . Thus, there is a skill gap of 1 , and the employee will require training. It is often the case that the employee requires training in more than one skill. This is exemplified by assigning Employee 2 to Task 3. A summarization of this information can also be found in the technical paper by Elmes, Evans, and DePuy (2008). 


\section{LITERATURE REVIEW}

Worker assignment and scheduling problems are faced by virtually all companies. For some companies, it is a simple scheduling problem. For example, hospitals need to schedule enough nurses to cover all patients. The major challenge with this scheduling problem is the length of shifts, when to take breaks, etc. Many manufacturing companies have the challenge of scheduling trained workers to specific jobs. In these instances, the workers are frequently cross-trained and can be assigned to a variety of different tasks.

The general worker assignment problem has been addressed in literature many times. Mazolla and Neebe (1986) developed a branch and bound algorithm to solve the general assignment problem where each potential assignment carries a cost. Even for a simple assignment problem where the only consideration is cost, the formulation is NP-complete and is extremely difficult to solve optimally when using a large data set (over 1,000 workers and 1,000 tasks). For this reason, Mazolla and Neebe developed a branch and bound algorithm to solve the problem.

More recent articles have expanded upon this general assignment problem. For example, Nembhard and Osothsilp (2005) incorporated individual learning and forgetting rates for workers. They concluded it is beneficial to schedule tasks with lower redundancy in order to reduce forgetting. That is, by giving workers a variety of tasks (instead of performing the same tasks repeatedly), workers are less likely to forget what they have learned. Sayin and Karabati (2007) took this one step further by including skill gaps into their formulation. They used a learning curve to model how workers improve their skill levels. 
Adding these layers of complexity improves the assignments generated by the model; unfortunately, it also makes it more difficult to solve optimally. Considering the size of many of these problems, heuristics are the only feasible way to generate a solution. For this particular problem, Table I below shows the number of decision variables and constraints for certain problem sizes.

TABLE I

SAMPLE PROBLEM SIZES

\begin{tabular}{|c|c|c|c|c|}
\hline workers & skills & tasks & decision variables & constraints \\
\hline 8 & 6 & 10 & 1328 & 552 \\
\hline 8 & 15 & 10 & 3200 & 1332 \\
\hline 15 & 6 & 20 & 2640 & 1925 \\
\hline 15 & 15 & 20 & 6150 & 4728 \\
\hline
\end{tabular}

Even with a relatively small problem size, the number of decision variables and constraints grows very rapidly. When dealing with very large data sets (over 1,000 workers), the amount of decision variables and constraints make it extremely time consuming to solve optimally. Thus, a heuristic is necessary to solve the problem in a practical amount of time.

Other authors have pointed out that these heuristic methods provide very good results when compared to optimum methods. Kolisch and Hartman (2005) as well as Campbell and Diaby (2002) analyzed several different heuristic techniques and showed that proper heuristics perform very well when compared to the optimum solution. In fact, heuristics are consistently withing $5 \%$ of optimum solutions and many times within $2 \%$. 
The model developed in this paper is both multi-objective and has a very large data set (over 1,000 workers and over 1,000 tasks). Thus, a heuristic is the only feasible way to solve the problem. However, "no matter how effective a heuristic or algorithm is, there remains a need to use human judgment to find a balance between an organization's conflicting objectives" (Belton and Elder, 1996). In multi-objective formulations, this "human judgment" is incorporated through weights attached to each objective function or through a value function. Cowling et. al.(2006) showed that this is not a good method since "objective weights are seldom known in advance" for complex real-world problems. Furthermore, it is unreasonable to expect the user to decide these weights without a thorough knowledge of the mathematical formulation (which a supervisor will not have). As an alternative, the program developed in this paper utilizes iterative input from the user to generate a solution. 


\section{FORMULATION OF THE PROBLEM}

\section{A. Single Objective Function Formulation}

This problem was previously solved according to one objective function: to minimize training costs (Douglas, 2006). The formulation of this problem can be found below:

\section{Parameters:}

$\{\mathrm{j}\}=$ set of skills needed to perform task $\mathrm{j}$

$\mathrm{S}_{\mathrm{ik}}=$ worker i's skill level for skill $\mathrm{k}$

$R_{\mathrm{jk}}=$ required skill level for task j's skill $\mathrm{k}$

$\mathrm{T}_{\mathrm{j}}=$ length $(\mathrm{hr})$ of task $\mathrm{j}$

$\mathrm{A}_{\mathrm{i}}=$ capacity $(\mathrm{hr})$ of worker $\mathrm{i}$

$\mathrm{C}_{\mathrm{klm}}=$ cost associated with raising a worker's skill level on skill $\mathrm{k}$ from level 1 to level $\mathrm{m}$ $\mathrm{E}_{\mathrm{klm}}=$ time required (hr) to raise a worker's skill level on skill $\mathrm{k}$ from level 1 to level $\mathrm{m}$

\section{Decision Variables:}

$\mathrm{X}_{\mathrm{ij}}= \begin{cases}1 & \text { workeri assigned to task } \mathrm{j} \\ 0 & \text { otherwise }\end{cases}$

$\mathrm{Z}_{\mathrm{ikS}} \mathrm{S}_{\mathrm{ik}} \mathrm{m}= \begin{cases}1 & \text { workeri receives training on skill } \mathrm{k} \text { to raise skill level from } \mathrm{S}_{\mathrm{ik}} \text { to } \mathrm{m} \\ 0 & \text { otherwise }\end{cases}$ $\mathrm{N}_{\mathrm{ik}}= \begin{cases}1 & \text { workeri does not need further training in skill } \mathrm{k} \\ 0 & \text { otherwise }\end{cases}$

\section{Objective Function:}

Minimize Training Cost

$$
\text { Minimize } Z_{1}=\sum_{i} \sum_{k} \sum_{m} C_{k S_{i k} m} Z_{i k S_{i k} m}
$$

\section{Constraints:}


Determine Needed Training $\quad S_{i k} N_{i k}+\sum_{m>S_{i k}}^{5} m Z_{i k S_{k} m} \geq R_{j k} X_{i j} \quad \forall i, j, k \in\{j\}$

$$
N_{i k}+\sum_{m>S_{i k}}^{5} Z_{i k S_{i k} m}=1
$$

All tasks assigned

$$
\sum_{i} X_{i j}=1
$$

All workers assigned at least one task

$$
\sum_{j} X_{i j} \geq 1
$$

Worker Capacity

$$
\sum_{j} T_{j} X_{i j}+\sum_{k} \sum_{m} E_{k S_{i k} m} Z_{i k S_{i k} m} \leq A_{i}
$$

Binary Variables

$$
X_{i j} \in\{0,1\}, \quad Z_{i k S_{i k} m} \in\{0,1\}, \quad N_{i k} \in\{0,1\} \quad \forall i, j, k, m(7)
$$

The objective function (1), as previously mentioned, is to minimize the total training cost. The inequalities (2) and (3) are used to determine the total amount of training a worker will need in order to meet the skill levels required by a certain task. Equation (4) is used to make sure that all tasks have been assigned and inequality (5) ensures that each worker is assigned at least one task. Inequality (5) could be removed from the formulation if it was not a requirement to retain the current workforce. Inequality (6) is used to enforce the worker capacity constraint. That is, training time plus the time required to complete the task(s) cannot exceed the total amount of time available to the worker. Lastly, line (7) defines all decision variables to be binary.

When dealing with a problem size of thousands of workers and thousands of tasks, solving this formulation optimally is not computationally feasible. Thus, DePuy et. al. 
(2006) developed the Meta-RaPS heuristic to solve this formulation on the large scale. Other authors such as Fowler, Wirojanagud, and Gel (2008) and Hartmann and Kolisch (2000) have shown that heuristics provide accurate results using far less computational time.

\section{B. Extension to Multi-Objective Formulation}

There are two primary reasons to expand this problem to a multi-objective formulation:

1) It may be beneficial to provide workers with more than the minimal amount of training.

2) In order to include worker preference in training decisions.

The expectation is that considering additional objective functions will improve the quality of the solution presented by the Decision Support System created to solve this worker assignment problem. Much of the information presented in this section is also summarized in the technical paper written by Elmes, Evans, and DePuy (2008).

As with most multi-objective models, it is very common for the objectives to be conflicting (Cowling et. al., 2006). This is very evident with the multi-objective formulation used in this model. Specifically, minimizing total training cost and maximizing the number of skill levels gained are conflicting objectives. The solution which is preferred by the Decision Maker will most likely not be the optimal solutions to one of the objective functions. Instead, the preferred solution will be a compromise of each objective function. 


\section{Maximize Total Training Received}

While minimizing the total training cost has obvious short-sighted benefits, it can actually create the same problem in the future that is currently being solved. The problem is that the workforce is undertrained. In addition, newer and newer technology is being implemented which magnifies the problem of an undertrained workforce. Thus, providing workers with minimal training today will lead to undertrained workers in the future as well. With that in mind, an objective function to maximize training received was included in the model:

Maximize Training $\quad$ Maximize $Z_{2}=\sum_{i} \sum_{k} \sum_{k^{\prime} \geq S_{i k}+1} \mathbf{\ell}^{\prime}-S_{i k} \bar{Z}_{i k S_{i k} k^{\prime}}$

The objective function above will maximize the total number of skill levels gained across all skills and all workers.

\section{Maximize Worker Satisfaction}

Numerous articles have been written highlighting the benefits of worker happiness. There is, obviously, the intrinsic benefit of having a happy workforce. There are also several benefits to the employer which can be seen on the bottom line. According to Sayin and Karabati (2007), "cross-training and skill improvement have been regarded as positive aspects for the self-esteem of the workers and their psychological well-being, which in turn would lead to higher productivity." Even as far back as 1919, George Bell recognized that if "we are to arrive at any real agreement between management and workers to cooperate in increasing production we must conceive of the master aim of the plant as being such production as is compatible with a real and measurable degree of 
human happiness and content in the work." Furthermore, Fischer and Sousa-Poza (2009) showed that "improvements in job satisfaction over time appear to prevent workers from (further) health deterioration."

With the addition of one new parameter $\mathrm{P}_{\mathrm{ik}}$ (defined below), worker preference regarding which skills they learn can be incorporated into the model. Sayin and Karabati (2007) showed that skill acquisition improves worker self-esteem. This model takes that one step further by allowing the workers to specify which skills they wish to acquire. The new parameter and formulation for this objective function can be found below:

Maximize Worker Satisfaction:

$$
\begin{aligned}
& \text { Maximize } Z_{3}=\sum_{i} \sum_{k} P_{i k} \sum_{k^{\prime} \geq S_{i k}+1} \mathbb{1}^{\prime}-S_{i k} \underline{Z}_{i k S_{i k} k^{\prime}} \\
& \mathrm{P}_{\mathrm{ik}}= \begin{cases}1 & \text { workeri would like additional training in skill } \mathrm{k} \\
0 & \text { otherwise }\end{cases}
\end{aligned}
$$

This objective function is very similar to (8), with the exception that each skill level gained is multiplied by $\mathrm{P}_{\mathrm{ik}}$. Thus, the objective function will maximize skill level gains in skills which the worker would prefer to be trained. While (8) seeks to maximize training across all workers and all skills, (9) incorporates worker preference regarding which skills they wish to gain expertise.

As an example, suppose that worker 5 currently has a training level of 2 on skill 9 (i.e. $\mathrm{S}_{59}=2$ ), but has not expressed an interest in additional training in that skill (i.e., $\mathrm{P}_{59}$ $=0$ ); then, a solution in which worker 5 were to be trained to level 4 on skill 9 (i.e. $Z_{5924}=$ 
1) would add $4-2=2$ to the second objective function $Z_{2}(8)$, but 0 to the third objective function $\mathrm{Z}_{3}(9)$.

\section{Goal of the Multi-Objective Decision Support System}

Simply put, the goal of this Decision Support System (DSS) is to provide the Decision Maker (DM) with a tool that is easy to use and will incorporate the expertise of the DM in order to find the preferred solution to the worker assignment problem. While Douglas (2006) solved this problem in terms of lowest training cost, it is believed that using a multi-objective approach will present a solution which is more preferred by the DM. 


\section{SOLUTION PROCESS}

It is important to point out an inherent problem with any multi-objective solution. In actuality, there is no solution which optimizes all objectives simultaneously. Instead, considering multiple objectives at the same time creates a Pareto-front where we have numerous good solutions (Cowling et. al., 2006). How then, do you decide which solution is the best compromise of all the objectives?

It is a long-standing industrial engineering principle that the person who knows best is the person who does the job every day. No good industrial engineer would begin the redesign of a process without talking to the worker who performs that process on a daily basis. With that in mind, the ideal way to select the best compromise solution is to use the knowledge of the person who has been doing the scheduling on a daily basis. This person is usually a supervisor and is referred to in this paper as the Decision Maker (DM).

The solution process used in the Decision Support System (DSS) developed in this paper differs from the solution process used in many Multi-Objective Optimization problems in the past. In these examples, a weight is assigned to each of the individual objective functions and then the problem is solved in one pass. The problem with this process is that the weight assigned to each individual objective function is essentially an arbitrary value selected by the Decision Maker (DM). That is, without a thorough knowledge of how the program runs one cannot assign accurate weights to each objective

function. Without accurate weights, the solution provided will not accurately reflect the 
DM's idea of a "good" solution. Cowling et. al. showed that the error associated with having inaccurate weights on the objective functions can be very costly.

The solution process used for this DSS does not use arbitrary weights assigned to each individual objective function. Instead, the expertise of the DM is used in an iterative fashion in order to guide the DSS to the best compromise solution. Since the DM will not have a detailed knowledge of the mathematical formulation behind the DSS, it is much easier to guide the program through iterative input as opposed to assigning weights to each objective function or by defining a value function.

\section{A. Multiple Stage Decision Support System}

The Decision Support System (DSS) is divided up into two primary stages (Figure 2). The first stage is to solve each of the three objective functions independent of one another. Each objective function is solved using the Meta-RaPS process developed by DePuy et. al. (2006) Meta-RaPS is employed because the computational time is too large to solve these objective functions optimally when using a large data set (over 1,000 workers and tasks).

The Decision Maker (DM) is presented a configurable number of solutions for each of the three objective functions-usually this is five. Solving each objective function is considered a step of the first stage. Since each objective function was solved independent of the others, the solutions presented to the DM are extremes and none of them are likely what the DM considers to be the best compromise solution. At this point, the DM selects 
one of the 15 solutions presented as the preferred solution of that group. This concludes Stage 1.

Stage 2 utilizes iterative input from the DM to execute compromise programming. This allows the DM to guide the DSS towards the best compromise solution. Using the solution identified in Stage 1, the DM is then asked two questions: which objective function should be improved; and how much should the other two be compromised? The DSS utilizes Meta-RaPS again to make trade-offs of worker-task assignments. The DSS will continue to make these trade-offs in order to improve the objective function identified by the DM until the compromise threshold (also identified by the DM) has been met. At this point, the new solution is presented to the DM. If the DM feels that this is the best compromise solution, the DSS is terminated and full results are presented to the DM. If not, Stage 2 is repeated and the DM is again 


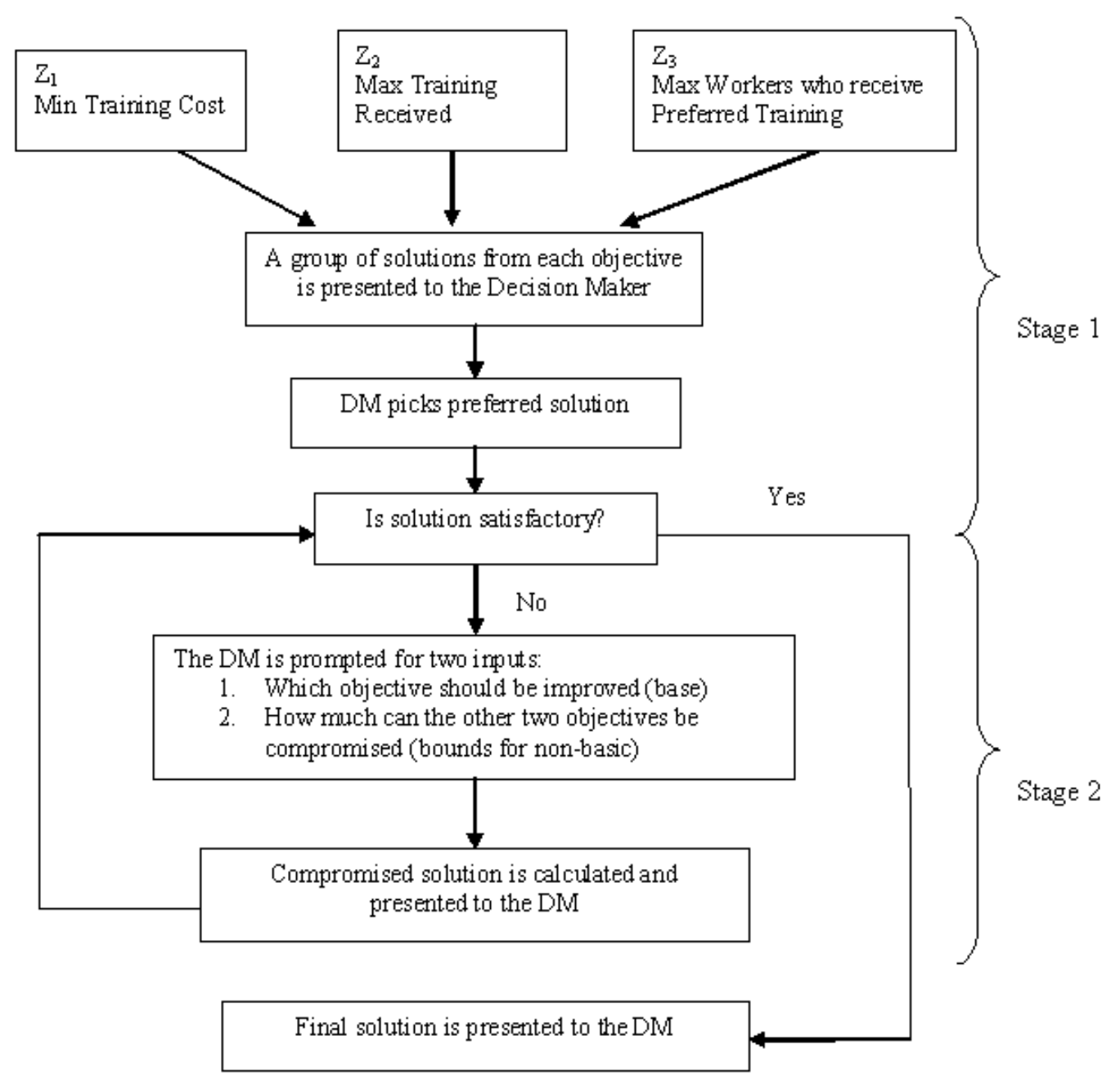

FIGURE 2 - Flowchart of Solution Process

Stage 2 is repeated until the DM has guided the DSS to the solution which he feels is the best compromise solution. This is the final solution, which is presented to the DM. 


\section{PSEUDO-CODE AND INPUT FROM THE USER}

The previous sections outlined the motivation for solving this large worker-task assignment problem in addition to providing a flowchart for the solution process. This section describes the VBA program that the Decision Maker (DM) uses as well as the pseudo-code for the problem solving algorithm. As mentioned before, a modified version of the Meta-RaPS algorithm developed by DePuy et. al. is used during each step.

The program (Decision Support System, or DSS) has two different buttons on the input sheet that control the operations. The first button is the "input button" and the second is the "find assignments" button. The input button requires little explanation-it sets up the tables for the DM to input all the necessary data (worker skill levels, etc.). The "find assignments" button actually executes the algorithm. This algorithm has been explained in more detail in previous sections-Figure 2 provides a good summary.

\section{A. Input Button}

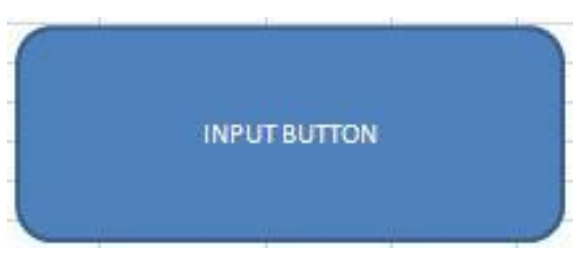

FIGURE 3 - Input Button

The first button is fairly self-explanatory: it will set up the input sheet for the DM to input values. When the user clicks on "input button," this will clear the sheet of all information. So, it is important to note that if you do not want to lose the information currently on the sheet, do not click the input button unless the data has been saved 
somewhere else. Once the input button is clicked, the program will prompt the user for the following information:

- Number of workers in the problem size

- Number of tasks in the problem size

- Number of skills

- Number of solutions to store for each objective in Stage 1

With this information, the program automatically generates all the tables that need to be filled out by the user. These tables can be found below:

- Worker-Skill Matrix

- Task-Skill Matrix

- Task Time Table

- Worker Capacity Table

- Cost to Train Matrix (cost of raising a skill level for each skill)

- Time to Train Matrix (again, for each skill level of each skill)

- Worker Preference Matrix

All of these tables are extremely important and must be filled out accurately by the DM. Obviously, this is the input data for the program, and without good input data, one cannot expect a good solution. The screenshot below shows a portion of the tables the DM fills out. In this example (same example used throughout this section), there are 5 workers, 5 skills, and 5 tasks: 


\begin{tabular}{|c|c|c|c|c|c|}
\hline \multicolumn{6}{|c|}{ Worker Skill Matrix } \\
\hline & Skill 1 & Skill 2 & Skill 3 & Skill 4 & Skill 5 \\
\hline Worker 1 & 2 & 4 & 4 & 3 & 4 \\
\hline Worker 2 & 3 & 2 & 3 & 4 & 3 \\
\hline Worker 3 & 3 & 5 & 4 & 5 & 4 \\
\hline Worker 4 & 4 & 3 & 2 & 2 & 2 \\
\hline Worker 5 & 3 & 3 & 3 & 3 & 2 \\
\hline \multicolumn{6}{|c|}{ Task Skill Matrix } \\
\hline & Skill 1 & Skill 2 & Skill 3 & Skill 4 & Skill 5 \\
\hline Task 1 & 4 & 4 & 1 & 1 & 2 \\
\hline Task 2 & 1 & 1 & 4 & 4 & 1 \\
\hline Task 3 & 3 & 3 & 1 & 1 & 1 \\
\hline Task 4 & 1 & 2 & 1 & 4 & 1 \\
\hline Task 5 & 1 & 4 & 4 & 1 & 3 \\
\hline
\end{tabular}

FIGURE 4 - Portion of Input Matrix

B. Find Assignments Button

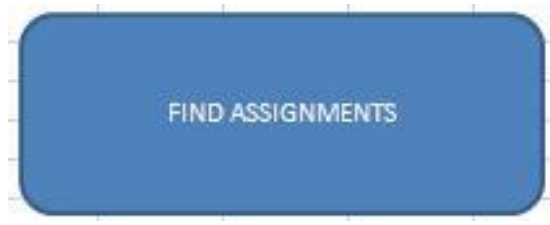

FIGURE 5 - Find Assignments Button

This button executes a very short program which actually calls upon four larger subprograms. The subprograms, in order, are listed below:

- InitialTrainingCostSoln (Stage 1, Step 1)

- InitialSkillGapSoln (Stage 1, Step 2)

- InitialWorkerPrefSoln (Stage 1, Step 3)

- Compromise (Stage 2)

The main program calls each subprogram individually in order to complete the algorithm. The first three subprograms comprise Stage 1-Find Initial Solutions. Each subprogram runs the Meta-RaPS heuristic to quickly calculate good solutions in regards to each objective function. Each subprogram then displays these solutions on the screen 
before moving on to the next subprogram. In this manner, the first three subprograms are extremely similar.

After running the three steps in stage 1, the list of initial solutions are presented to the DM. The DM chooses one of these initial solutions as the "best" from that group. However, as discussed earlier, all of the initial solutions optimize one of the objective functions without consideration of the others. Thus, it is unlikely the solution chosen at this point will be the final solution. This solution is simply used as a starting point moving into Stage 2.

The screenshot below continues the example setup earlier with 5 workers, 5 skills, and 5 tasks. In this example, the DM has chosen one of the solutions to $\mathrm{Z}_{2}$ (Maximize Training). In turn, $\mathrm{Z}_{1}$ (Minimize Training Cost) and $\mathrm{Z}_{3}$ (Maximize Worker Satisfaction) are far from optimized.

\begin{tabular}{|c|c|}
\hline Iteration & 1 \\
\hline Worker & Task \\
\hline 1 & 3 \\
\hline 2 & 1 \\
\hline 3 & 4 \\
\hline 4 & 2 \\
\hline 5 & 5 \\
\hline Total Cost & 57 \\
\hline Total Training Amount & 11 \\
\hline Total Worker Preference & 7 \\
\hline
\end{tabular}

FIGURE 6 - Initial Solution (Stage 1 complete)

Unlike the first three subprograms, the final subprogram (Stage 2) utilizes iterative input from the DM. Stage 2 uses compromise programming in order to improve one of the objective functions at the sacrifice of the other two. The DM is first prompted for which objective function should be improved. Since the solution used to initialize Stage 
2 was one that optimized $\mathrm{Z}_{2}$ then the available choices are $\mathrm{Z}_{1}$ or $\mathrm{Z}_{3}$. The screenshot below shows this prompt:

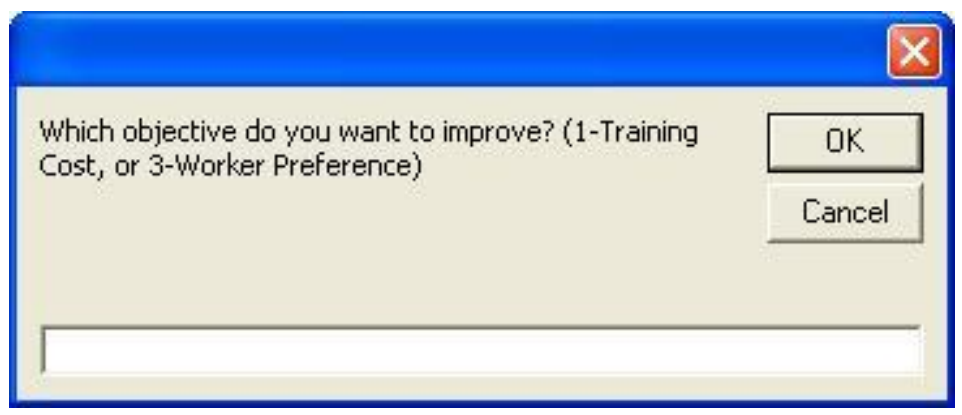

FIGURE 7 - Which Objective to Improve

After the DM specifies which objective function to improve, the DM inputs a percentage that the other two objective functions can be sacrificed. The screenshot for this choice is below:

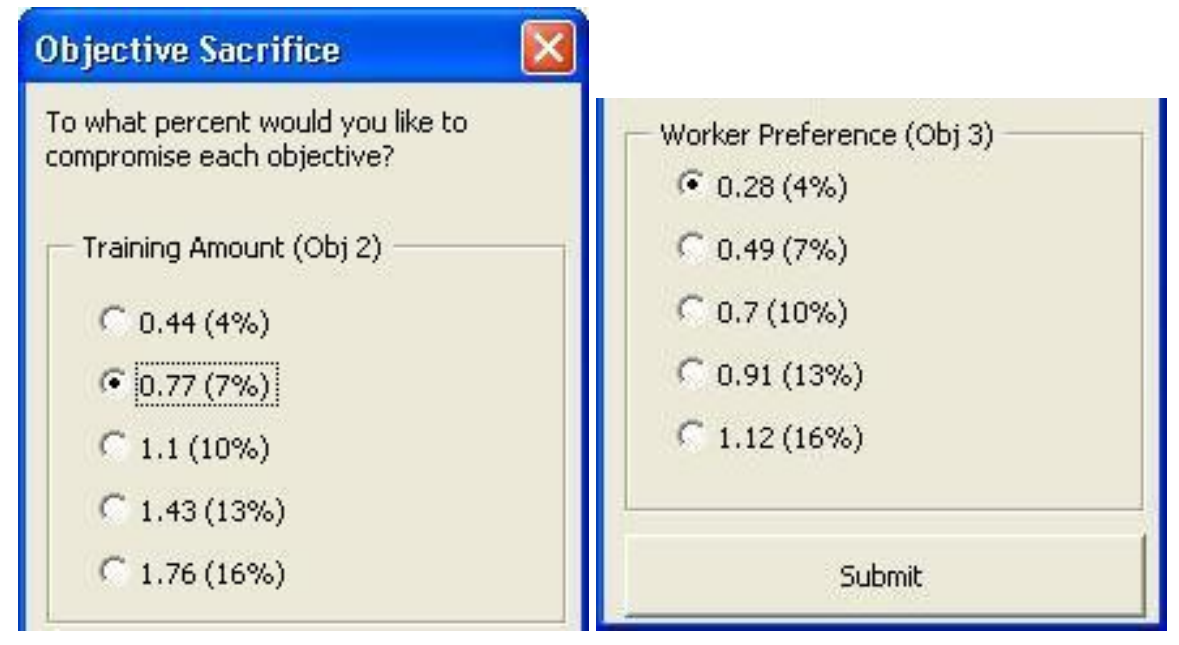

FIGURE 8 - How Much to Sacrifice Each Objective

At this point, the algorithm now has a complete solution to work with in addition to trade-off data between the different objectives provided by the DM. With this information, a series of worker-task assignments swaps are made in order to improve the base objective. The subprogram will continue to make swaps until one (or both) of the 
bounds have been violated or there are no more beneficial swaps. The new solution is then presented to the DM:

\begin{tabular}{|l|r|r|r|r|}
\hline Iteration & & 1 & & 2 \\
\hline Worker & 1 & 3 & 1 & 3 \\
\hline & 2 & 1 & 2 & 2 \\
\hline & 3 & 4 & 3 & 4 \\
\hline & 4 & 2 & 4 & 1 \\
\hline Total Cost & 5 & 5 & 5 & 5 \\
\hline Total Training Amount & & 57 & & 34 \\
\hline Total Worker Preference & 11 & & 6 \\
\hline
\end{tabular}

FIGURE 9 - Solution After Compromise Programming

At this point, if the DM is satisfied with the solution, the program is terminated. If the DM is not satisfied, the program will prompt the DM for new trade-off data and repeat Stage 2. This is repeated until the DM has reached the best compromise solution.

It is important to point out again that there is no solution which will optimize all the objective functions simultaneously (Cowling et. al., 2006). Generally speaking, if a multi-objective problem is solved optimally, it is through the use of weights on each objective function or through a value function. It can be very difficult for the DM to specify weights or define a value function. This is because the DM is selected for his expertise in the production setting-not his knowledge of mathematical formulations. The goal of this program is to use iterative input from the DM to guide the algorithm towards the best compromise solution. This is a much more intuitive way for the DM to use the program. 


\section{Pseudo-code for Decision Support System (DSS)}

This section summarizes the code used for the DSS. There are four primary sections of code to analyze. One section for each of the three objective functions and the fourth section is for the compromise programming.

\section{1. $\quad$ Key Variables used in Pseudo-code}

Before jumping into the pseudo-code, it is beneficial to review some of the key variables used throughout the code. First of all, it is important to note the similarities between the three steps in the first stage. Take the first step for example; many of the decisions about worker-task assignments are based on the variables 'totaltaskcost' and 'totalworkercost.' These variables are calculated using the 'workertaskcost' variable, which is a large array that holds the cost associated with training any worker to any task. So, 'totaltaskcost' is simply the sum of all workertaskcost values for a specific task. Similarly, 'totalworkercost' is the sum of all workertaskcost values for a specific worker. These 'total' variables are used to determine who is the least trained worker (maxcostworker, for phase 1) and which is the most difficult task (maxcosttask, for phase 2). More detail on how the worker-task assignments are made can be found in the following section. The intention of this section is to bring attention to the similarities between the three steps in the first stage.

In Stage 1, Step 1, the program uses variables such as 'totaltaskcost,' 'workertaskcost,' etc. as outlined above. For Step 2, the program uses variables such as 'totaltasksgap' and 'workertasksgap.' Similarly, for Step 3, the program uses variables such as 'totaltaskpref' and 'workertaskpref.' Each step uses the "total" variables 
associated with the objective function for making worker-task assignments; however, it does keep track of all "total" variables (i.e. the ones for the other objective functions). While the other "total" variables are not used for determining worker-task assignments, they are used to calculate the objective function values once a complete assignment has been made. Also, these values are used in Stage 2, where certain objectives are being compromised for the benefit of another.

\section{Generalized Meta-RaPS Pseudo-code}

The pseudo-code below shows the generalized form of the Meta-RaPS heuristic. Each of the three initial solution programs outlined below follows this generalized code:

Do Until max number of iterations is reached

Do Until all tasks are assigned

Identify the greedy assignment

If random value $<=$ priority percentage Then

Make greedy assignment

Else

Develop a candidate list of other assignments close in value to the greedy assignment End If

Choose an assignment from the candidate list

Loop

Place the solution in its ranked position compared to all other iterations Loop

Sections 3-5 show how the Meta-RaPS heuristic is applied to each objective function. Meta-RaPS is actually run twice for each objective function-once to make sure each worker is assigned one task and the second time to assign all remaining tasks. If there is not a constraint to retain the current workforce, the program can jump straight to the second use of Meta-RaPS and simply assign all tasks.

\section{InitialTrainingCostSoln Pseudo-code}


Once the DM clicks the "find assignments" button, this is the first subprogram which is run. This subprogram uses the Meta-RaPS heuristic to determine worker-task assignments. The pseudo-code for this subprogram is below:

Do Until max number of iterations is reached

Do Until each worker has a task assigned

Identify the maxcostworker

Identify mincosttask for maxcostworker

If random value $<=$ priority percentage Then

Assign mincosttask to maxcostworker

Else

If worker is unassigned and totalworkercost $>=$ maxcostworker* restriction percent Then

If task is unassigned and cost to assign worker to that task $<=$ mincost $*(1+$ restriction percentage $)$ Then End If

This worker-task assignment goes on candidate list

End If

Use random number to select a worker-task assignment from the candidate list

End If

Loop

Do Until all tasks are assigned

Identify maxcosttask

Identify mincostworker

If random value $<=$ priority percentage Then

Assign mincostworker to maxcosttask

Else

If task is unassigned and totaltaskcost $>=$ maxcosttask*restriction percent Then

If cost to assign worker to that task $<=$ mincost*(1+restriction percentage $)$ Then End If

This worker-task assignment goes on candidate list

End If

Use a random worker to select a worker-task assignment from the candidate list

End If

Loop

Update objective function values and worker skill levels

Eliminate the solution if it is a duplicate from a previous iteration

Place the solution in its ranked position compared to all other iterations 
The complete code can be found in Appendix E. The two phases for the above section of code are very similar. They both use the Meta-RaPS heuristic, which is a modified greedy algorithm. The first step is to identify the worker who has the highest training cost across all tasks. Next, the program identifies the task which can be assigned to that worker at the minimum cost. This would be the greedy assignment, and according to Meta-RaPS, this assignment is sometimes made. However, Meta-RaPS differs from a greedy algorithm because it sometimes makes an assignment from a "candidate list." The candidate list contains other worker-task assignments that are within a certain percentage of the greedy assignment. This selection is made using random numbers.

Phase 1 continues assigning tasks to workers who are currently unassigned. Once each worker has a task assignment, the program moves to phase 2, where the rest of the tasks are assigned. Phase 2 is extremely similar to phase 1 since they both employ the Meta-RaPS heuristic. The difference is that phase 1 identifies a worker for assignment and then finds a task for that worker. On the other hand, phase 2 identifies a task first, and then finds a worker to assign to that task. Once phase 2 is complete, a complete worker-task assignment has been completed. Next, the program determines how good the current solution is compared to solutions from other iterations.

The first step in determining how the current solution compares to other iterations is to check if the current solution is a duplicate solution. All duplicate solutions are thrown out and a new iteration begins. However, considering the problem size and the randomness of Meta-RaPS, there are not many duplicate solutions. If a unique solution is generated, the program determines what rank the solution is. If it is not a top ranking 
solution (the number of solutions to store is a user input), then the program moves to the next iteration. If the current solution is a unique, top ranking solution, then it is saved in a best solution matrix. Once all the iterations are complete, the top ranking solutions are printed on the screen and the program moves on to the second subprogram (Stage 1, Step 2): InitialSkillGapSoln.

\section{InitialSkillGapSoln Pseudo-code}

The program automatically starts this sub-program after the first sub-program (InitialTrainingCostSoln; Stage 1, Step 1) is completed. The pseudo-code for this subprogram is below:

Do Until max number of iterations is reached

Do Until each worker has a task assigned

Identify the maxsgapworker Identify maxsgaptask for maxsgapworker If random value $<=$ priority percentage Then

Assign maxsgaptask to maxsgapworker

Else

If worker is unassigned and totalworkersgap >= maxsgapworker*restriction percent Then

If task is unassigned and the skill gap closed >= maxsgap*restriction percent Then

This worker-task assignment goes on candidate list

End If End If

Use random number to select a worker-task assignment from the candidate list

End If

Loop

Update objective function values and worker skill levels

Do Until all tasks are assigned

Identify maxsgaptask

Identify maxsgapworker

If random value <= priority percentage Then

Assign maxsgapworker to maxsgaptask

Else 
If task is unassigned and totaltasksgap $>=$ maxsgaptask*restriction percent Then

If skill level gained $>=$ maxsgap*restriction percent Then

This worker-task assignment goes on candidate list

End If End If

Use a random worker to select a worker-task assignment from the candidate list

End If

Loop

Update objective function values and worker skill levels

Eliminate the solution if it is a duplicate from a previous iteration

Place the solution in its ranked position compared to all other iterations

Loop

Where the first sub-program identified the least trained worker (according to training costs), and assigned that worker the most inexpensive task, this sub-program is actually maximizing the total number of skill levels gained. So, the greedy assignment would be the worker with the fewest total skill levels and the task which would gain that worker the most skill levels. The candidate list is comprised of other worker-task assignments that increase the worker's skill levels within a certain percentage of the greedy assignment.

This sub-program is very similar to the first one (and the last one). This subprogram has two phases which both employ Meta-RaPS. Also, this subprogram uses the same ranking system. The major difference between each sub-program is that each one solves a different objective function.

Once this sub-program is complete, it will print the top results on the screen and then automatically move on to the last sub-program in Stage 1: InitialWorkerPrefSoln.

\section{InitialWorkerPrefSoln Pseudo-code}


The program automatically starts this sub-program after the first sub-program (InitialTrainingCostSoln; Stage 1, Step 1) is completed. The pseudo-code for this subprogram is below:

Do Until max number of iterations is reached

Do Until each worker has a task assigned

Identify the maxprefworker

Identify maxpreftask for maxprefworker

If random value $<=$ priority percentage Then

Assign maxpreftask to maxprefworker

Else

If worker is unassigned and totalworkerpref $>=$ maxprefworker*restriction percent Then

If task is unassigned and the skill levels gained $*$ worker preference $>=$ maxsgap*restriction percent Then End If

This worker-task assignment goes on candidate list

End If

Use random number to select a worker-task assignment from the candidate list

End If

Loop

Update objective function values and worker skill levels

Do Until all tasks are assigned

Identify maxpreftask

Identify maxprefworker

If random value $<=$ priority percentage Then

Assign maxprefworker to maxpreftask

Else

If task is unassigned and totaltaskpref $>=$ maxpreftask $*$ restriction percent Then

If skill level gained $*$ worker preference $>=$ maxpref*restriction percent Then End If

This worker-task assignment goes on candidate list

End If

Use a random worker to select a worker-task assignment from the candidate list

End If

Loop

Update objective function values and worker skill levels

Eliminate the solution if it is a duplicate from a previous iteration

Place the solution in its ranked position compared to all other iterations 
This subprogram is extremely similar to the previous: InitialSkillGapSoln. The worker-task assignments are still determined using the amount of skill levels gained on a particular task; however, for this objective function, the skill levels gained is also multiplied by a binary worker preference value. Consider the following hypothetical example: if worker 1 is assigned to task 1, he will gain 3 skill levels in both skills 1 and 2 . In addition, worker 1 has a preference value of 0 for skill 1 and 1 for skill 2 . That is, he does not have a desire to learn skill 1, but does want to learn skill 2. In step 2, where preferences are not considered, both skills would contribute a value of 3 to the objective function if this assignment was selected. However, for step 3, the skill levels gained is multiplied by the preference value. Thus, skill 1 would contribute 0 to the objective function since the worker preference value is 0 also. On the other hand, skill 2 would still add 3 to the objective function since the preference value is 1 for that skill. Here is another situation to consider in understanding the relationship between step 2 and 3 . If the worker preference matrix was all set to 1 , then the objective function for step 2 and 3 would be equivalent.

The results from this subprogram are displayed on the screen for the DM to reference along with the other two subprograms. Complete worker-task assignments are printed on the screen; however, for large data sets, it is not feasible to compare the different solutions based on the individual assignments. For this reason, there is a summary at the top of the page which simply shows the three objective function values associated with each initial solution. The DM is then prompted to identify which of the initial solutions is the preferred solution. At this point, the program is using input from 
the DM to help guide the algorithm towards a solution which best matches the preferences of the DM.

This concludes the first stage of the program. Next, the program automatically moves into stage 2 - Compromise Programming.

\section{Compromise Programming Pseudo-code}

Compromise Programming makes up the second stage of the program, and is iterative in nature. At this point, the DM has identified a single solution to use in the second stage. Two pieces of information are prompted from the DM:

- Which objective function to improve

- Bounds on the other objective functions

Using this information, the program will continue to swap worker-task assignments until one of the bounds on the other objective functions have been violated. The pseudo-code for stage 2 is below:

Do Until usersatisfied $=1$

Prompt User for which objective to improve

Option Box used to input bounds on the other two objective functions

For $\mathrm{i}=1$ to numworkers

For $\mathrm{j}=1$ to numtasks

If worker $\mathrm{i}$ is assigned to task $\mathrm{j}$ Then

For i2 = 1 to numworkers

For $\mathrm{j} 2=1$ to numworkers

Calculate the affect this swap has on base and store than in a matrix

Next j2

Next i2

End If

Next $j$

Next $i$ 
Do Until bound on either non-basic objective function is violated Or no swap improves base

Determine which swap will increase the base the most and make that swap Update worker skill levels and objective function values

Loop

Print the new solution on the screen

Ask if the user is satisfied (usersatisfied=[1 for yes, 0 for no $]$ )

Loop

Right before the final loop, the program asks if the DM is satisfied with the current solution. If he is not, then the subprogram loops to the top and asks the DM to specify a base and bounds on non-basic objective functions once again. Hence, the DM has complete control in manipulating the solution to accurately reflect his preferences. He can continue to select the same objective function as the base to improve it more, or he can select a different base to "guide" the program in another direction. Additionally, this goal is achieved without assigning weights to each objective function at the beginning of the program. Instead, the DM's knowledge and experience is used later in the program, and in a manner that is simple for the DM. After all, it is much easier for someone inexperienced with how the program runs to specify bounds on objective functions than to assign arbitrary weights at the beginning of the program. 


\section{RESULTS}

This section outlines the results calculated using several different data sets. All three objective functions are analyzed, though the Minimize Training Cost is done so in less detail due to the fact that Douglas (2006) already examined using Meta-RaPS on this objective function in great detail. Similarly, this paper did not analyze the different values that could be used for percent restriction and percent priority which could be used. These values are used when the program decides whether to make a greedy assignment or to choose an assignment from the candidate list. Douglas (2006) determined that 30 and 80 for percent restriction and priority, respectively, was best suited for this assignment problem. These values were used in this data analysis.

Since Meta-RaPS executes very quickly and is quite random, it is very beneficial to use a large number of iterations in order to find a near optimal solution. Douglas (2006) recommended using 5000 iterations. However, it was determined that a much smaller value is sufficient (200 iterations). While the program is running, the user can see the top list of solutions currently found. Meta-RaPS is surprisingly effective at finding a good solution quickly. Thus, a small number of iterations is sufficient. However, it should be noted that when used in a practical setting, it would be recommended to use a larger number of iterations to guarantee a good solution. For that matter, it would be wise to tweak each of the parameter settings depending on the specific application.

Microsoft VBA for Excel was used to program the Meta-RaPS algorithm. While there are other programming languages that would have been more efficient, Excel is commonly used in practical settings. Thus, it is a very familiar way for the user to input 
data into the model. In addition, since most companies already own and use Microsoft Excel, there is no need to purchase any additional software.

The optimum solutions were calculated using LINGO mathematical software. The code used can be found in Appendix E. The results for each objective function are outlined in the following subsections.

\section{A. Minimize Training Cost Results}

Table II summarizes the results for the Minimize Training Cost objective function. The full results can be found in Appendix A. Run times are in seconds unless otherwise noted.

\section{TABLE II}

MINIMIZETRAINING COST RESULTS

\begin{tabular}{|r|r|r|r|r|r|l|l|}
\hline workers & skills & tasks & Meta-RaPS & run-time & Optimum & run-time & \%diff \\
\hline 8 & 6 & 10 & 121.6 & 8.5 & 116.5 & 12.9 & $4.2 \%$ \\
\hline 8 & 15 & 10 & 532.6 & 8.1 & 513 & $1.75 \mathrm{hr}$ & $3.8 \%$ \\
\hline 15 & 6 & 20 & 220.7 & 12.4 & 172 & $31.5 \mathrm{hr}$ & $28.3 \%$ \\
\hline 15 & 15 & 20 & 801 & 11.56 & - & - & - \\
\hline
\end{tabular}

When using a small data set, Meta-RaPS is extremely effective at finding a close to optimum solution. However, once a larger data set was tested, Meta-RaPS was on average close to $30 \%$ worse than optimum. Obviously, it would be preferred to have a solution closer to optimum. This is where raising the number of iterations would be beneficial. These trial runs were used with only 200 iterations. This is reflected in the very fast run times. Certainly, a run time of 12.4 seconds is greatly superior to a run time of over 31 hours (the 15 worker, 6 skills data set). Using a larger number of iterations 
would produce results closer to optimum and still have a much shorter run time than the optimum method.

\section{B. Maximize Skill Levels Gained Results}

Table III summarizes the results for the Maximize Skill Levels Gained objective function. The full results can be found in Appendix B. Run times are in seconds unless otherwise noted.

TABLE III

MAXIMIZE SKILL LEVELS GAINED RESULTS

\begin{tabular}{|r|r|r|r|r|r|r|l|}
\hline workers & skills & tasks & Meta-RaPS & run-time & Optimum & run-time & \%diff \\
\hline 8 & 6 & 10 & 53.4 & 13.3 & 63.4 & 1 & $15.8 \%$ \\
\hline 8 & 15 & 10 & 128.9 & 9.0 & 147 & 7 & $12.3 \%$ \\
\hline 15 & 6 & 20 & 108.7 & 14.5 & 138 & 2 & $21.2 \%$ \\
\hline 15 & 15 & 20 & 233.1 & 13.5 & 294 & 409 & $20.7 \%$ \\
\hline
\end{tabular}

It can be seen from the table that Meta-RaPS produces solutions which are close to optimum. While a little more than $20 \%$ from optimum may seem like a significant difference, this is in part because only 200 iterations were used. A larger number of iterations would produce a solution closer to the optimum.

However, there is an important trade-off between the number of iterations to use and the run-time of the program. LINGO is able to produce optimum results for this objective function (as well as Maximize Worker Preference) much faster than the Minimize Training Cost objective function. Thus, it is not as easy to simply state that raising the iterations will solve the problem. However, on the other hand, Meta-RaPS 
already produces solutions closer to optimum compared to the first objective function. So, there is not as much a need to raise the number of iterations.

\section{Maximize Worker Preference Results}

Table IV summarizes the results for the Maximize Worker Preference objective function. The full results can be found in Appendix C. Run times are in seconds unless otherwise noted.

\section{TABLE IV}

\section{MAXIMIZE WORKER PREFERENCE RESULTS}

\begin{tabular}{|r|r|r|r|r|r|r|l|}
\hline workers & skills & tasks & Meta-RaPS & run-time & Optimum & run-time & \%diff \\
\hline 8 & 6 & 10 & 30.6 & 14.6 & 39.2 & 1.1 & $21.9 \%$ \\
\hline 8 & 15 & 10 & 76.0 & 9.6 & 96 & 1 & $20.8 \%$ \\
\hline 15 & 6 & 20 & 66.9 & 14.2 & 98 & 2 & $31.8 \%$ \\
\hline 15 & 15 & 20 & 139.2 & 13.8 & 187 & 81 & $25.6 \%$ \\
\hline
\end{tabular}

This objective function is very similar in nature to the Maximize Skill Levels Gained objective function. However, you can see from the percent difference, that Meta-RaPS was not as effective with this objective function. On the other hand, Meta-RaPS still generated solutions closer to optimum than the first objective function (Minimize Total Cost). This is a promising result and indicates that Meta-Raps is an effective method for generating solutions to all three objective functions.

An interesting thing to point out with this data set is the run-time for calculating the optimum solution. When comparing the optimum solution to Meta-RaPS run time for the first objective function (total cost), the optimum solution took over 31 hours compared to only 12.4 seconds for Meta-RaPS. There is nowhere near the same disparity when we 
look at the results for this objective function. At the same time, the optimum solution did take more than five times as long (81 seconds) compared to Meta-RaPS (13.8) seconds. When using an extremely large data set (over 1,000 workers), this difference in run-time will be very beneficial.

\section{Compromise Programming Results}

Unfortunately, there is no easy way to analyze results for the second stage of the program. The only way to determine an optimum solution would be to run the model through LINGO. However, to do that, each of the three objective functions would need to have a weight assigned to it or a value function is neccesary. The premise to this paper is to avoid using those techniques. Instead, the knowledge and expertise of the DM guides the program to the best compromise solution. In a way, this interaction does assign weights to each objective function, but it is done in a more intuitive fashion. This will help the DM more accurately weigh the importance of each objective function.

This idea is supported by Cowling et. al.(2006) as well as Belton and Elder (1996). They point out that using inaccurate weights can have very costly effects on the quality of the solution. Since this program is designed for a supervisor in the field to use, it can be assumed that the user will not have a detailed knowledge of the mathematical formulation and thus cannot assign accurate weights. This is by design. Instead, the user can guide the program towards the best compromise solution using the knowledge he has gained in the field. 


\section{CONCLUSIONS AND RECOMMENDATIONS}

The goal of this paper is to extend the Decision Support System (DSS) developed by Douglas (2006) to a multi-objective formulation. The two objective functions to maximize skill levels gained as well as to maximize worker preference were incorporated in with the original objective function to minimize total cost. Clearly, the objective function to maximize skill levels gained contradicts the original objective function to minimize cost because training costs money. However, as Cowling et. al.(2006) pointed out, virtually every real world multi-objective problem has "many and contradictory objectives." How then, do we determine the best solution?

It is a long-held Industrial Engineering principle that the person who knows how to do the job the best is the person who has been doing it the longest. Enter: the Decision Maker (DM). The Decision Support System (DSS) developed in this paper is designed to take advantage of the knowledge the DM possesses from working in the field for many years. Hence, the logical selection for the DM is a supervisor.

Many multi-objective formulations in the past assign a weight to each objective function in order to solve the formulation as one larger optimization problem. Cowling et. al.(2006) show that the cost of using inaccurate weights in this type of formulation can have "severe" detrimental effects. For this program, we assume that the DM will not be able to assign accurate weights to the objective functions because this person does not have a deep understanding of the mathematical formulation - that is not the DM's purpose. The DM is being selected because of his knowledge of the production setting 
(worker competencies, creating schedules, etc.). Thus, the DM's knowledge is used in an iterative manner to arrive at the best compromise solution in the eyes of the DM.

One thing that makes this program unique is that it is designed to be used for very large problem sizes (thousands of workers). This presents a problem regarding solution time. Instead of solving each of the three objective functions optimally, they are solved using the Meta-RaPS heuristic developed by DePuy, et. al. (2003) and adapted by Douglas (2006) to be used for the assignment problem. Solving each objective function independently is stage 1 of the program. These solutions (a configurable number) are then fed into stage 2 of the program - which incorporates input from the DM.

Once a pool of initial solutions has been generated, the DM selects the one he feels is closest to the "best" solution. From there, the program utilizes compromise programming to adjust the solution as the DM sees fit. More specifically, the DM identifies which objective function should be improved and then provides bounds on the other objective functions. The program then makes trade-offs in the worker-task assignments in order to improve the identified objective function at the cost of the other. This process can be repeated as many times as necessary until the DM has a solution he is satisfied with. In this manner, the DM is essentially assigning weights to each objective function. These weights are determined through the DM's iterative input regarding trade-off values.

While this concept appears to be very effective, it has yet to be tested using realworld data. There are several other areas that provide good opportunity for future research. For example, the VBA code was written by an Industrial Engineer-not a computer programmer. As a result, the code is very inefficient, and cleaning up the code 
would most likely reduce computational times. This could be done through code refactoring. Code refactoring would shorten the length of the code as well as remove unnecessary steps. A shorter run time would have huge benefits in practical applications.

Another area for improvement is in the compromise programming. Currently, when the DM is specifying how much to sacrifice the objective functions (for the benefit of another) these ranges are predefined percentages. It is possible to program this so that the DM can enter his preferred percentage instead of choosing one from the option list. Another way to get this input from the DM would be to include a slider. This way the DM could move the slider to the percentage that he prefers. This provides more flexibility while still maintaining ease of use.

One downside to the current input sheet is that it is very time consuming to enter in data. When there are over 1,000 workers and tasks, it will take the DM a very long time to input skill levels for each worker and task. There may be a way to group certain workers together (by department, by years of experience, etc.). This way, the DM could input a smaller amount of data. Similarly, there may be a way to group tasks together in order to reduce input time.

Lastly, another area for improvement is with the parameters used in the program. As mentioned during the Results section, Douglas (2006) concluded that the best values for percent restriction and percent priority were 30 and 80, respectively. These values were only tested for the first objective function (Minimize Training Cost). However, these values were also used for the two new objective functions introduced in this paper. The results could have been more accurate it different parameters were used for these 
objective functions. On the other hand, this is somewhat a moot point because the parameters for all three objective functions would need to be validated for a real-world problem. 


\section{LIST OF REFERENCES}

Bell, George. 1919. Production the Goal. Annals of the American Academy of Political and Social Science. Vol. 85, Modern Manufacturing. A Partnership of Idealism and Common Sense, 1-7

Belton, V., Elder, M.D. 1996. Exploring a Multicriteria Approach to Production Scheduling. The Journal of the Operational Research Society. Volume 47, Issue 1, $162-174$

Campbell, G.M., Diaby, M. 2002. Development and Evaluation of an Assignment Heuristic for Allocating Cross-trained Workers. European Journal of Operational Research. Volume 138, Issue 1, 9-20

Cowling, P., Colledge, N., Keshav, D., Remde, S. 2006. The Trade Off between Diversity and Quality for Multi-objective Workforce Scheduling. Lecture Notes in Computer Science, Volume 3906/2006, 13-24

DePuy, G.W., Moraga, R., Whitehouse, G. 2003. Meta-RaPS: A simple and effective approach for solving the traveling salesman problem. Transportation Research Part E. 212 .

Douglas, Allison M. 2006. A Modified Greedy Algorithm for the Task Assignment Problem. Master of Engineering Thesis, University of Louisville.

Elmes, B.B., Evans, G.W., DePuy, G.W., 2008. A Multi-Objective Decision Support System for Workforce Training. Proceedings of the 2008 Industrial Engineering Research Conference

Fischer, J., Sousa-Poza, A. 2009. Does Job Satisfaction Improve the Health of Workers? New Evidence Using Panel Data and Objective Measures of Health. Health Economics, Volume 18, Issue 1, 71-89

Fowler, J., Wirojanagud, P., Gel, E. 2008. Heuristics for workforce planning with worker differences. European Journal of Operational Research. Volume 190, Issue 3, 724740 .

Hartmann, S., Kolisch, R. 2000. Experimental evaluation of state-of-the-art heuristics for the resource-constrained project scheduling problem. European Journal of Operational Research. Volume 127, Issue 2, 394-407

Kolisch, R., Hartmann, S. 2005. Experimental investigation of heuristics for resourceconstrained project scheduling: An update. European Journal of Operational Research. Volume 174, Issue 1, 23-37

Nembhard, D.A., Osothsilp, N. 2005. Learning and Forgetting-Based Worker Selection for Tasks of Varying Complexity. The Journal of the Operational Research Society Volume 56, Issue 5,576-587 
Mazolla, J.B., Neebe, A.W. 1986. Resource-Constrained Assignment Scheduling. Operations Research. Volume 34, Issue 4, 560-572

Sayin, S., Karabati, S. 2007. Assigning cross-trained workers to departments: A twostage optimization model to maximize utility and skill improvement. European Journal of Operational Research. Volume 176, Issue 3, 1643-1658 


\section{APPENDIX A—Results for Minimize Total Training Cost}

The table below has the full results for the Minimize Training Cost objective function. The majority of the calculations were performed on a desktop computer with an Intel Pentium 4 3.0GHz processor with $1 \mathrm{~GB}$ of ram. Some of the run times had to be discarded because they were performed on a laptop with faulty hardware that skewed the run times.

For each trial run, the data was randomly generated. Summaries of this data can be found the Results section of the text.

\begin{tabular}{|c|c|c|c|c|c|c|c|c|}
\hline Trial & Workers & Skills & Tasks & $\begin{array}{l}\text { Meta- } \\
\text { RaPS }\end{array}$ & $\begin{array}{l}\text { run- } \\
\text { time }\end{array}$ & Optimum & $\begin{array}{l}\text { run- } \\
\text { time }\end{array}$ & \%diff \\
\hline 1 & 8 & 6 & 10 & 70 & 8.3 & 70 & 2 & $0.0 \%$ \\
\hline 2 & 8 & 6 & 10 & 94 & & 87 & 13 & $8.0 \%$ \\
\hline 3 & 8 & 6 & 10 & 95 & & 91 & 3 & $4.4 \%$ \\
\hline 4 & 8 & 6 & 10 & 161 & 11.4 & 161 & 21 & $0.0 \%$ \\
\hline 5 & 8 & 6 & 10 & 82 & & 77 & 12 & $6.5 \%$ \\
\hline 6 & 8 & 6 & 10 & 102 & & 94 & 5 & $8.5 \%$ \\
\hline 7 & 8 & 6 & 10 & 145 & & 142 & & $2.1 \%$ \\
\hline 8 & 8 & 6 & 10 & 128 & & 128 & 8 & $0.0 \%$ \\
\hline 9 & 8 & 6 & 10 & 69 & & 67 & 6 & $3.0 \%$ \\
\hline 10 & 8 & 6 & 10 & 171 & & 163 & & $4.9 \%$ \\
\hline 11 & 8 & 6 & 10 & 124 & 8.2 & 112 & 10 & $10.7 \%$ \\
\hline 12 & 8 & 6 & 10 & 153 & 8.4 & 153 & 18 & $0.0 \%$ \\
\hline 13 & 8 & 6 & 10 & 152 & & 145 & 23 & $4.8 \%$ \\
\hline 14 & 8 & 6 & 10 & 167 & 7.9 & 161 & 49 & $3.7 \%$ \\
\hline 15 & 8 & 6 & 10 & 120 & 7.6 & 109 & 4 & $10.1 \%$ \\
\hline 16 & 8 & 6 & 10 & 112 & 7.6 & 104 & 6 & $7.7 \%$ \\
\hline 17 & 8 & 15 & 10 & 513 & 7.8 & 513 & $6377 \mathrm{~s}$ & $0.0 \%$ \\
\hline 18 & 8 & 15 & 10 & 514 & 8.2 & & & $0.2 \%$ \\
\hline 19 & 8 & 15 & 10 & 548 & 8 & & & $6.8 \%$ \\
\hline 20 & 8 & 15 & 10 & 545 & 7.9 & & & $6.2 \%$ \\
\hline 21 & 8 & 15 & 10 & 518 & 7.9 & & & $1.0 \%$ \\
\hline 22 & 8 & 15 & 10 & 546 & 7.9 & & & $6.4 \%$ \\
\hline
\end{tabular}




\begin{tabular}{|c|c|c|c|c|c|c|c|c|}
\hline 23 & 8 & 15 & 10 & 533 & 8 & & & $3.9 \%$ \\
\hline 24 & 8 & 15 & 10 & 528 & 10 & & & $2.9 \%$ \\
\hline 25 & 8 & 15 & 10 & 536 & 7.9 & & & $4.5 \%$ \\
\hline 26 & 8 & 15 & 10 & 548 & 7.9 & & & $6.8 \%$ \\
\hline 27 & 8 & 15 & 10 & 528 & 7.9 & & & $2.9 \%$ \\
\hline 28 & 8 & 15 & 10 & 529 & 8 & & & $3.1 \%$ \\
\hline 29 & 8 & 15 & 10 & 532 & 8 & & & $3.7 \%$ \\
\hline 30 & 8 & 15 & 10 & 528 & 7.9 & & & $2.9 \%$ \\
\hline 31 & 8 & 15 & 10 & 528 & 7.9 & & & $2.9 \%$ \\
\hline 32 & 8 & 15 & 10 & 548 & 7.9 & & & $6.8 \%$ \\
\hline 33 & 15 & 6 & 20 & 223 & 12.4 & 172 & $31.5 \mathrm{hr}$ & $29.7 \%$ \\
\hline 34 & 15 & 6 & 20 & 211 & 11.9 & & & $22.7 \%$ \\
\hline 35 & 15 & 6 & 20 & 208 & 12 & & & $20.9 \%$ \\
\hline 36 & 15 & 6 & 20 & 220 & 12 & & & $27.9 \%$ \\
\hline 37 & 15 & 6 & 20 & 223 & 11.8 & & & $29.7 \%$ \\
\hline 38 & 15 & 6 & 20 & 204 & 11.8 & & & $18.6 \%$ \\
\hline 39 & 15 & 6 & 20 & 220 & 11.9 & & & $27.9 \%$ \\
\hline 40 & 15 & 6 & 20 & 237 & 12.1 & & & $37.8 \%$ \\
\hline 41 & 15 & 6 & 20 & 209 & 12.9 & & & $21.5 \%$ \\
\hline 42 & 15 & 6 & 20 & 224 & 11.8 & & & $30.2 \%$ \\
\hline 43 & 15 & 6 & 20 & 234 & 11.8 & & & $36.0 \%$ \\
\hline 44 & 15 & 6 & 20 & 232 & 11.8 & & & $34.9 \%$ \\
\hline 45 & 15 & 6 & 20 & 234 & 11.9 & & & $36.0 \%$ \\
\hline 46 & 15 & 6 & 20 & 222 & 13.2 & & & $29.1 \%$ \\
\hline 47 & 15 & 6 & 20 & 232 & 11.9 & & & $34.9 \%$ \\
\hline 48 & 15 & 6 & 20 & 206 & 13 & & & $19.8 \%$ \\
\hline 49 & 15 & 6 & 20 & 223 & 11.9 & & & $29.7 \%$ \\
\hline 50 & 15 & 6 & 20 & 224 & 16.7 & & & $30.2 \%$ \\
\hline 51 & 15 & 6 & 20 & 220 & 11.9 & & & $27.9 \%$ \\
\hline 52 & 15 & 6 & 20 & 225 & 12 & & & $30.8 \%$ \\
\hline 53 & 15 & 6 & 20 & 202 & 15.4 & & & $17.4 \%$ \\
\hline 54 & 15 & 6 & 20 & 218 & 11.9 & & & $26.7 \%$ \\
\hline 55 & 15 & 6 & 20 & 224 & 12 & & & $30.2 \%$ \\
\hline 56 & 15 & 15 & 20 & 778 & 11.7 & N/A & $\mathrm{N} / \mathrm{A}$ & \\
\hline 57 & 15 & 15 & 20 & 814 & 11.7 & & & \\
\hline 58 & 15 & 15 & 20 & 778 & 11.4 & & & \\
\hline 59 & 15 & 15 & 20 & 795 & 11.5 & & & \\
\hline 60 & 15 & 15 & 20 & 770 & 11.7 & & & \\
\hline 61 & 15 & 15 & 20 & 807 & 11.6 & & & \\
\hline 62 & 15 & 15 & 20 & 820 & 11.4 & & & \\
\hline 63 & 15 & 15 & 20 & 777 & 11.5 & & & \\
\hline
\end{tabular}




\begin{tabular}{|l|l|l|l|l|l|l|l|l|}
64 & 15 & 15 & 20 & 800 & 11.6 & & & \\
\hline 65 & 15 & 15 & 20 & 773 & 11.5 & & & \\
\hline 66 & 15 & 15 & 20 & 821 & 11.5 & & & \\
\hline 67 & 15 & 15 & 20 & 848 & 11.6 & & & \\
\hline 68 & 15 & 15 & 20 & 792 & 11.7 & & & \\
\hline 69 & 15 & 15 & 20 & 809 & 11.5 & & & \\
\hline 70 & 15 & 15 & 20 & 813 & 11.5 & & & \\
\hline 71 & 15 & 15 & 20 & 811 & 11.6 & & & \\
\hline 72 & 15 & 15 & 20 & 811 & & & & \\
\hline
\end{tabular}


APPENDIX B — Results for Maximize Skill Levels Gained

The table below has the full results for the Maximize Skill Levels Gained objective function. The calculations were performed on a desktop computer with an Intel Pentium 4 3.0GHz processor with $1 \mathrm{~GB}$ of ram.

\begin{tabular}{|r|r|r|r|r|r|r|r|l|}
\hline Trial & Workers & Skills & Tasks & \multicolumn{1}{l|}{$\begin{array}{l}\text { Reta- } \\
\text { RaPS }\end{array}$} & $\begin{array}{l}\text { run- } \\
\text { time }\end{array}$ & $\begin{array}{l}\text { Optimum } \\
\text { time }\end{array}$ & \%diff \\
\hline 1 & 8 & 6 & 10 & 47 & 9.1 & 53 & 1 & $11.3 \%$ \\
\hline 2 & 8 & 6 & 10 & 52 & & 61 & 1 & $14.8 \%$ \\
\hline 3 & 8 & 6 & 10 & 51 & & 61 & 1 & $16.4 \%$ \\
\hline 4 & 8 & 6 & 10 & 53 & 12.5 & 64 & 1 & $17.2 \%$ \\
\hline 5 & 8 & 6 & 10 & 52 & & 63 & 1 & $17.5 \%$ \\
\hline 6 & 8 & 6 & 10 & 50 & & 56 & 1 & $10.7 \%$ \\
\hline 7 & 8 & 6 & 10 & 54 & & 68 & 1 & $20.6 \%$ \\
\hline 8 & 8 & 6 & 10 & 62 & & 75 & 1 & $17.3 \%$ \\
\hline 9 & 8 & 6 & 10 & 45 & & 58 & 1 & $22.4 \%$ \\
\hline 10 & 8 & 6 & 10 & 62 & & 70 & 1 & $11.4 \%$ \\
\hline 11 & 8 & 6 & 10 & 48 & 24.1 & 58 & 1 & $17.2 \%$ \\
\hline 12 & 8 & 6 & 10 & 53 & 22.3 & 63 & 1 & $15.9 \%$ \\
\hline 13 & 8 & 6 & 10 & 64 & & 73 & 1 & $12.3 \%$ \\
\hline 14 & 8 & 6 & 10 & 64 & 8.4 & 78 & 1 & $17.9 \%$ \\
\hline 15 & 8 & 6 & 10 & 52 & 8.4 & 59 & 1 & $11.9 \%$ \\
\hline 16 & 8 & 6 & 10 & 45 & 8.4 & 54 & 1 & $16.7 \%$ \\
\hline 17 & 8 & 15 & 10 & 129 & 8.7 & 147 & 7 & $12.2 \%$ \\
\hline 18 & 8 & 15 & 10 & 128 & 9.2 & & & $12.9 \%$ \\
\hline 19 & 8 & 15 & 10 & 129 & 9.1 & & & $12.2 \%$ \\
\hline 20 & 8 & 15 & 10 & 128 & 8.9 & & & $12.9 \%$ \\
\hline 21 & 8 & 15 & 10 & 131 & 9 & & & $10.9 \%$ \\
\hline 22 & 8 & 15 & 10 & 128 & 8.9 & & & $12.9 \%$ \\
\hline 23 & 8 & 15 & 10 & 131 & 8.9 & & & $10.9 \%$ \\
\hline 24 & 8 & 15 & 10 & 130 & 10.5 & & & $11.6 \%$ \\
\hline 25 & 8 & 15 & 10 & 130 & 8.9 & & & $11.6 \%$ \\
\hline 26 & 8 & 15 & 10 & 128 & 8.9 & & & $12.9 \%$ \\
\hline 27 & 8 & 15 & 10 & 128 & 8.8 & & & $12.9 \%$ \\
\hline 28 & 8 & 15 & 10 & 128 & 8.9 & & & $12.9 \%$ \\
\hline 29 & 8 & 15 & 10 & 129 & 8.9 & & & $12.2 \%$ \\
\hline 30 & 8 & 15 & 10 & 130 & 9.2 & & & $11.6 \%$ \\
\hline
\end{tabular}




\begin{tabular}{|c|c|c|c|c|c|c|c|c|}
\hline 31 & 8 & 15 & 10 & 129 & 8.9 & & & $12.2 \%$ \\
\hline 32 & 8 & 15 & 10 & 126 & 8.9 & & & $14.3 \%$ \\
\hline 33 & 15 & 6 & 20 & 108 & 13.5 & 138 & 2 & $21.7 \%$ \\
\hline 34 & 15 & 6 & 20 & 110 & 13.8 & & & $20.3 \%$ \\
\hline 35 & 15 & 6 & 20 & 107 & 13.8 & & & $22.5 \%$ \\
\hline 36 & 15 & 6 & 20 & 107 & 13.8 & & & $22.5 \%$ \\
\hline 37 & 15 & 6 & 20 & 111 & 13.7 & & & $19.6 \%$ \\
\hline 38 & 15 & 6 & 20 & 108 & 13.9 & & & $21.7 \%$ \\
\hline 39 & 15 & 6 & 20 & 108 & 15.7 & & & $21.7 \%$ \\
\hline 40 & 15 & 6 & 20 & 108 & 13.8 & & & $21.7 \%$ \\
\hline 41 & 15 & 6 & 20 & 109 & 14.3 & & & $21.0 \%$ \\
\hline 42 & 15 & 6 & 20 & 109 & 16.1 & & & $21.0 \%$ \\
\hline 43 & 15 & 6 & 20 & 109 & 13.7 & & & $21.0 \%$ \\
\hline 44 & 15 & 6 & 20 & 110 & 13.8 & & & $20.3 \%$ \\
\hline 45 & 15 & 6 & 20 & 109 & 13.8 & & & $21.0 \%$ \\
\hline 46 & 15 & 6 & 20 & 111 & 14.3 & & & $19.6 \%$ \\
\hline 47 & 15 & 6 & 20 & 108 & 13.9 & & & $21.7 \%$ \\
\hline 48 & 15 & 6 & 20 & 110 & 13.7 & & & $20.3 \%$ \\
\hline 49 & 15 & 6 & 20 & 109 & 13.5 & & & $21.0 \%$ \\
\hline 50 & 15 & 6 & 20 & 109 & 24.8 & & & $21.0 \%$ \\
\hline 51 & 15 & 6 & 20 & 109 & 13.7 & & & $21.0 \%$ \\
\hline 52 & 15 & 6 & 20 & 108 & 13.7 & & & $21.7 \%$ \\
\hline 53 & 15 & 6 & 20 & 108 & 14.7 & & & $21.7 \%$ \\
\hline 54 & 15 & 6 & 20 & 108 & 14 & & & $21.7 \%$ \\
\hline 55 & 15 & 6 & 20 & 108 & 13.7 & & & $21.7 \%$ \\
\hline 56 & 15 & 15 & 20 & 235 & 13.7 & 294 & 409 & $20.1 \%$ \\
\hline 57 & 15 & 15 & 20 & 234 & 13.6 & & & $20.4 \%$ \\
\hline 58 & 15 & 15 & 20 & 236 & 13.5 & & & $19.7 \%$ \\
\hline 59 & 15 & 15 & 20 & 234 & 13.6 & & & $20.4 \%$ \\
\hline 60 & 15 & 15 & 20 & 233 & 13.7 & & & $20.7 \%$ \\
\hline 61 & 15 & 15 & 20 & 232 & 13.4 & & & $21.1 \%$ \\
\hline 62 & 15 & 15 & 20 & 233 & 13.2 & & & $20.7 \%$ \\
\hline 63 & 15 & 15 & 20 & 232 & 13.7 & & & $21.1 \%$ \\
\hline 64 & 15 & 15 & 20 & 232 & 13.6 & & & $21.1 \%$ \\
\hline 65 & 15 & 15 & 20 & 230 & 13.5 & & & $21.8 \%$ \\
\hline 66 & 15 & 15 & 20 & 235 & 13.6 & & & $20.1 \%$ \\
\hline 67 & 15 & 15 & 20 & 230 & 13.4 & & & $21.8 \%$ \\
\hline 68 & 15 & 15 & 20 & 234 & 13.4 & & & $20.4 \%$ \\
\hline 69 & 15 & 15 & 20 & 234 & 13.7 & & & $20.4 \%$ \\
\hline 70 & 15 & 15 & 20 & 234 & 13.5 & & & $20.4 \%$ \\
\hline 71 & 15 & 15 & 20 & 232 & 13.4 & & & $21.1 \%$ \\
\hline
\end{tabular}




\begin{tabular}{|l|l|l|l|l|l|l|l|}
72 & 15 & 15 & 20 & 232 & & & \\
\hline
\end{tabular}


APPENDIX C—Results for Maximize Worker Preference

The table below has the full results for the Maximize Worker Preference objective function. The calculations were performed on a desktop computer with an Intel Pentium $43.0 \mathrm{GHz}$ processor with $1 \mathrm{~GB}$ of ram.

\begin{tabular}{|r|r|r|r|r|r|r|r|l|}
\hline Trial & Workers & Skills & Tasks & \multicolumn{1}{l|l}{$\begin{array}{l}\text { Reta- } \\
\text { RaPS }\end{array}$} & $\begin{array}{l}\text { run- } \\
\text { time }\end{array}$ & $\begin{array}{l}\text { Optimum } \\
\text { time }\end{array}$ & \%diff \\
\hline 1 & 8 & 6 & 10 & 17 & 9.7 & 24 & 1 & $29.2 \%$ \\
\hline 2 & 8 & 6 & 10 & 29 & & 33 & 1 & $12.1 \%$ \\
\hline 3 & 8 & 6 & 10 & 29 & & 34 & 2 & $14.7 \%$ \\
\hline 4 & 8 & 6 & 10 & 37 & 12.9 & 44 & 1 & $15.9 \%$ \\
\hline 5 & 8 & 6 & 10 & 34 & & 42 & 1 & $19.0 \%$ \\
\hline 6 & 8 & 6 & 10 & 36 & & 43 & 1 & $16.3 \%$ \\
\hline 7 & 8 & 6 & 10 & 32 & & 41 & 1 & $22.0 \%$ \\
\hline 8 & 8 & 6 & 10 & 36 & & 49 & 1 & $26.5 \%$ \\
\hline 9 & 8 & 6 & 10 & 24 & & 37 & 1 & $35.1 \%$ \\
\hline 10 & 8 & 6 & 10 & 29 & & 42 & 1 & $31.0 \%$ \\
\hline 11 & 8 & 6 & 10 & 30 & 29.6 & 36 & 1 & $16.7 \%$ \\
\hline 12 & 8 & 6 & 10 & 30 & 23.8 & 39 & 1 & $23.1 \%$ \\
\hline 13 & 8 & 6 & 10 & 27 & & 35 & 1 & $22.9 \%$ \\
\hline 14 & 8 & 6 & 10 & 37 & 8.5 & 48 & 1 & $22.9 \%$ \\
\hline 15 & 8 & 6 & 10 & 33 & 9 & 39 & 1 & $15.4 \%$ \\
\hline 16 & 8 & 6 & 10 & 30 & 9 & 41 & 1 & $26.8 \%$ \\
\hline 17 & 8 & 15 & 10 & 76 & 9.3 & 96 & 1 & $20.8 \%$ \\
\hline 18 & 8 & 15 & 10 & 76 & 9.7 & & & $20.8 \%$ \\
\hline 19 & 8 & 15 & 10 & 77 & 9.6 & & & $19.8 \%$ \\
\hline 20 & 8 & 15 & 10 & 76 & 9.6 & & & $20.8 \%$ \\
\hline 21 & 8 & 15 & 10 & 76 & 9.5 & & & $20.8 \%$ \\
\hline 22 & 8 & 15 & 10 & 78 & 9.6 & & & $18.8 \%$ \\
\hline 23 & 8 & 15 & 10 & 75 & 9.5 & & & $21.9 \%$ \\
\hline 24 & 8 & 15 & 10 & 74 & 10.7 & & & $22.9 \%$ \\
\hline 25 & 8 & 15 & 10 & 78 & 9.6 & & & $18.8 \%$ \\
\hline 26 & 8 & 15 & 10 & 76 & 9.6 & & & $20.8 \%$ \\
\hline 27 & 8 & 15 & 10 & 74 & 9.6 & & & $22.9 \%$ \\
\hline 28 & 8 & 15 & 10 & 76 & 9.5 & & & $20.8 \%$ \\
\hline 29 & 8 & 15 & 10 & 76 & 9.6 & & & $20.8 \%$ \\
\hline 30 & 8 & 15 & 10 & 78 & 9.6 & & & $18.8 \%$ \\
\hline
\end{tabular}




\begin{tabular}{|c|c|c|c|c|c|c|c|c|}
\hline 31 & 8 & 15 & 10 & 77 & 9.5 & & & $19.8 \%$ \\
\hline 32 & 8 & 15 & 10 & 73 & 9.5 & & & $24.0 \%$ \\
\hline 33 & 15 & 6 & 20 & 65 & 13.4 & 98 & 2 & $33.7 \%$ \\
\hline 34 & 15 & 6 & 20 & 70 & 13.8 & & & $28.6 \%$ \\
\hline 35 & 15 & 6 & 20 & 69 & 13.8 & & & $29.6 \%$ \\
\hline 36 & 15 & 6 & 20 & 68 & 13.7 & & & $30.6 \%$ \\
\hline 37 & 15 & 6 & 20 & 67 & 13.9 & & & $31.6 \%$ \\
\hline 38 & 15 & 6 & 20 & 68 & 13.8 & & & $30.6 \%$ \\
\hline 39 & 15 & 6 & 20 & 66 & 15.9 & & & $32.7 \%$ \\
\hline 40 & 15 & 6 & 20 & 68 & 13.9 & & & $30.6 \%$ \\
\hline 41 & 15 & 6 & 20 & 65 & 13.8 & & & $33.7 \%$ \\
\hline 42 & 15 & 6 & 20 & 67 & 15.8 & & & $31.6 \%$ \\
\hline 43 & 15 & 6 & 20 & 66 & 14 & & & $32.7 \%$ \\
\hline 44 & 15 & 6 & 20 & 66 & 13.8 & & & $32.7 \%$ \\
\hline 45 & 15 & 6 & 20 & 70 & 14.1 & & & $28.6 \%$ \\
\hline 46 & 15 & 6 & 20 & 65 & 13.8 & & & $33.7 \%$ \\
\hline 47 & 15 & 6 & 20 & 67 & 13.8 & & & $31.6 \%$ \\
\hline 48 & 15 & 6 & 20 & 67 & 13.9 & & & $31.6 \%$ \\
\hline 49 & 15 & 6 & 20 & 66 & 13.6 & & & $32.7 \%$ \\
\hline 50 & 15 & 6 & 20 & 66 & 17.3 & & & $32.7 \%$ \\
\hline 51 & 15 & 6 & 20 & 67 & 13.8 & & & $31.6 \%$ \\
\hline 52 & 15 & 6 & 20 & 67 & 14.3 & & & $31.6 \%$ \\
\hline 53 & 15 & 6 & 20 & 68 & 14 & & & $30.6 \%$ \\
\hline 54 & 15 & 6 & 20 & 63 & 14.1 & & & $35.7 \%$ \\
\hline 55 & 15 & 6 & 20 & 67 & 13.8 & & & $31.6 \%$ \\
\hline 56 & 15 & 15 & 20 & 144 & & 187 & 81 & $23.0 \%$ \\
\hline 57 & 15 & 15 & 20 & 135 & 13.8 & & & $27.8 \%$ \\
\hline 58 & 15 & 15 & 20 & 138 & 13.7 & & & $26.2 \%$ \\
\hline 59 & 15 & 15 & 20 & 137 & 13.6 & & & $26.7 \%$ \\
\hline 60 & 15 & 15 & 20 & 142 & 13.6 & & & $24.1 \%$ \\
\hline 61 & 15 & 15 & 20 & 139 & 14.2 & & & $25.7 \%$ \\
\hline 62 & 15 & 15 & 20 & 141 & 13.6 & & & $24.6 \%$ \\
\hline 63 & 15 & 15 & 20 & 137 & 13.8 & & & $26.7 \%$ \\
\hline 64 & 15 & 15 & 20 & 141 & 13.7 & & & $24.6 \%$ \\
\hline 65 & 15 & 15 & 20 & 142 & 13.7 & & & $24.1 \%$ \\
\hline 66 & 15 & 15 & 20 & 139 & 14.6 & & & $25.7 \%$ \\
\hline 67 & 15 & 15 & 20 & 135 & 13.9 & & & $27.8 \%$ \\
\hline 68 & 15 & 15 & 20 & 141 & 13.9 & & & $24.6 \%$ \\
\hline 69 & 15 & 15 & 20 & 142 & 13.9 & & & $24.1 \%$ \\
\hline 70 & 15 & 15 & 20 & 139 & 13.8 & & & $25.7 \%$ \\
\hline 71 & 15 & 15 & 20 & 139 & 13.8 & & & $25.7 \%$ \\
\hline
\end{tabular}




\begin{tabular}{|l|l|l|l|l|l|l|l|}
72 & 15 & 15 & 20 & 135 & & & \\
\hline
\end{tabular}




\section{APPENDIX D-LINGO CODE}

Below is the LINGO code used to calculate the optimum solutions in the Results section. All three objective functions are included in the code. Two of the three are commented out so that only one will run at a time. Simply changing which objective functions are commented will execute the correct objective function.

Model :

! Crane skills training;

Data :

$$
\begin{aligned}
& \text { numworkers }=\text { aOLE ('input 15-15-20.xls'); } \\
& \text { numskills }=\text { dOLE ('input 15-15-20.xls'); } \\
& \text { numtasks = dOLE('input 15-15-20.xls'); }
\end{aligned}
$$

\section{enddata}

\section{Sets:}

levels/1..5/;

workers/1..numworkers/;

skills/1..numskills/;

tasks/1..numtasks/;

incremental (skills, levels) : inctime, inccost;

workerskill (workers, skills) :wpref, wskill, notrai

$\mathrm{n}$;

taskskill (tasks,skills):tskill;

workertask (workers, tasks) : assigned;

tasktime (tasks): ttime;

workercap (workers) : wcap;

levelpairs (levels, levels);

training (skills, levels, levels) : tcost, trtime;

moretraining (workers, skills, levels, levels) :more train;

allcombos (workers, tasks, skills); 
Data:

wskill= @OLE('input 15-15-20.xls');
tskill $=$ @OLE('input 15-15-20.xls');
ttime = @OLE('input 15-15-20.xls');
wcap = @OLE('input 15-15-20.xls');
tcost = @OLE('input 15-15-20.xls');
trtime = @OLE('input 15-15-20.xls');
wpref = @OLE('input 15-15-20.xls');
Enddata

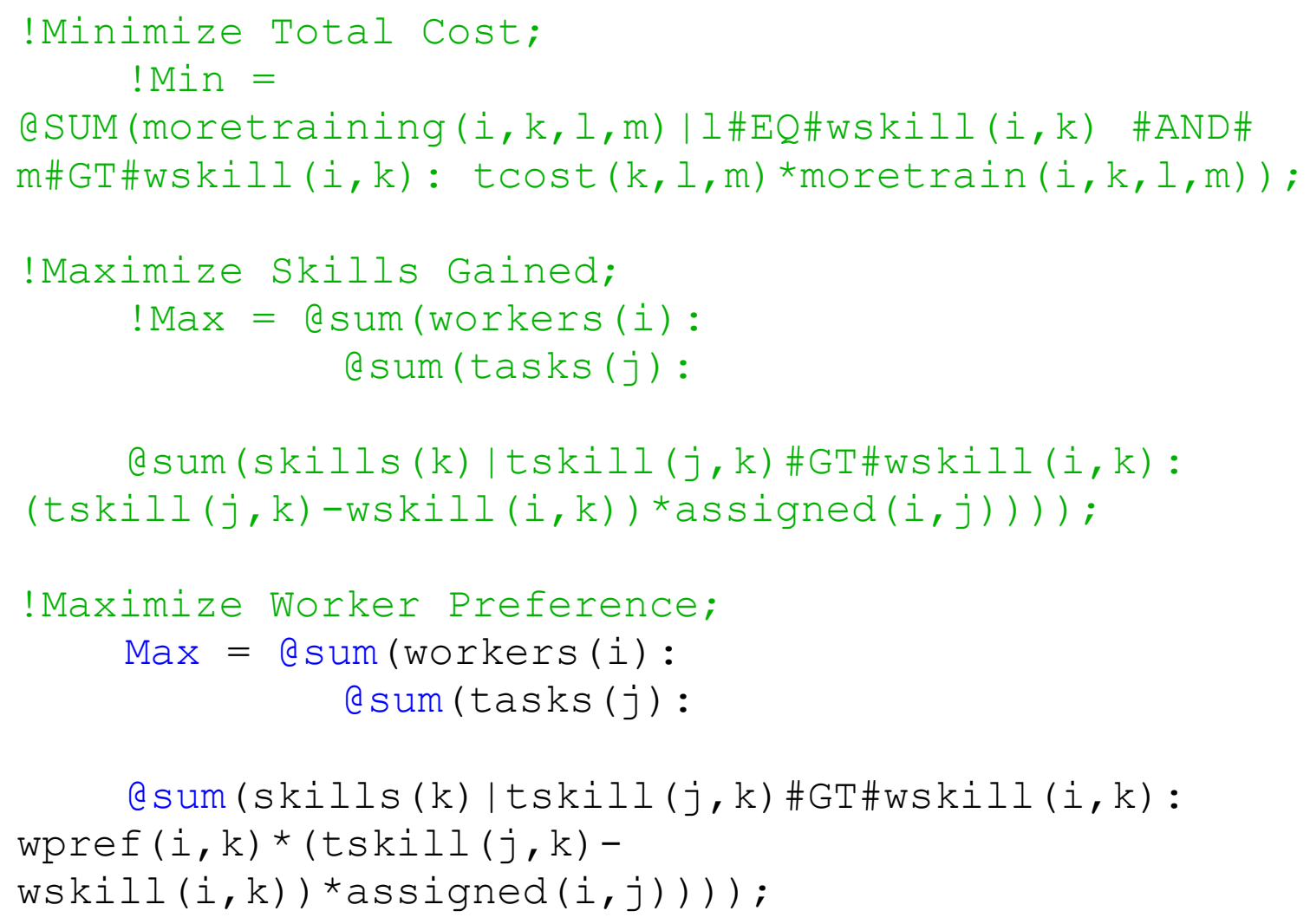


@FOR (workers (i):

@For (tasks $(j)$ :

@For (skills (k) |tskill (j,k) \#GT\#0:

wskill $(i, k) * \operatorname{notrain}(i, k)+$

(a) Sum (levels (m)|m\#GT\#wskill (i,k) :m*moretrain(i,k , $\operatorname{wskill}(i, k), m)) \quad>=\operatorname{tskill}(j, k) * a s s i g n e d(i, j))))$;

@FOR (workers (i):

@FOR (skills (k): notrain $(i, k)+$

(a) Sum(levels (m)|m\#GT\#wskill (i,k) :moretrain(i,k,w $\operatorname{skill}(i, k), m))=1)$ );

@FOR (tasks $(j):$

(a) Sum (workers (i) :assigned $(i, j))=1$ );

QFOR (workers (i):

QSum(tasks $(j)$ :assigned $(i, j))>=1)$;

@FOR (workers (i) :

(asum (tasks $(j)$ : ttime $(j)$ *assigned $(i, j))+$

@Sum (skills (k) : @sum(levels (m) :trtime (k, wskill (i , k) , m) *moretrain (i,k, wskill (i,k), m) )) <=wcap (i)) ;

@FOR (workers (i) :

(aFOR (tasks $(j)$ : @BIN (assigned $(i, j))))$;

@FOR (workers (i):

aFOR (skills (k): (aBIN (notrain $(i, k))))$;

@For (moretraining ( $i, k, l, m) \mid l \# E Q \# w s k i l l(i, k)$ : QBIN (moretrain $(i, k, 1, m)))$;

End 
APPENDIX E-

Code for Input Sheet

'This command button will setup the input sheet for the user

Public Sub InputButton_Click()

Dim numworkers As Single

Dim numskills As Single

Dim numtasks As Single

Dim numSolns As Single

Dim MinCostSwitch As Single

Dim MinSkillGapSwitch As Single

Dim MinPreferenceSwitch As Single

Dim MinOverTimeSwitch As Single

Dim TotalNumObjFn As Single

'initial values

TotalNumObjFn $=3$

MinOverTimeSwitch $=0$

'Clears all the sheets (except parameters)

Sheets("Input").Cells.Clear

Sheets("Results Summary").Cells.Clear

'Inputs setup values (number of workers/skills/tasks and which Objective Functions will be considered 
Sheets("Input").Select

numworkers = Application.InputBox("Input number of workers", "")

numskills = Application.InputBox("Input number of skills", "")

numtasks = Application.InputBox("Input number of tasks", "")

numSolns = Application.InputBox("Input number of initial solutions to store", "')

'Insert values

ActiveSheet.Cells $(1,1)$. Value $=$ "PROBLEM SIZE"

ActiveSheet.Cells $(2,1)$. Value $=$ "Number of workers"

ActiveSheet.Cells $(3,1)$. Value $=$ "Number of skills"

ActiveSheet.Cells $(4,1)$.Value $=$ "Number of tasks"

ActiveSheet.Cells $(5,1)$.Value $=$ "Numer of initial Solutions"

ActiveSheet.Cells $(6,1)$. Value = "OBJECTIVE FUNCTIONS"

ActiveSheet.Cells $(7,1)$.Value = "Min Training Cost?"

ActiveSheet.Cells $(8,1)$.Value = "Max Amount of Training?"

ActiveSheet.Cells $(9,1)$.Value = "Max Worker Preference?"

'ActiveSheet.Cells $(10,1)$.Value = "Min OverTime?"

ActiveSheet.Cells $(2,2)$. Value $=$ numworkers

ActiveSheet.Cells $(3,2)$.Value $=$ numskills

ActiveSheet.Cells $(4,2)$. Value $=$ numtasks

ActiveSheet.Cells $(5,2)$. Value $=$ numSolns

ActiveSheet.Cells $(7,2)$. Value $=$ MinCostSwitch

ActiveSheet.Cells $(8,2)$.Value $=$ MinSkillGapSwitch

ActiveSheet.Cells(9, 2).Value $=$ MinPreferenceSwitch

'ActiveSheet.Cells(10, 2).Value = MinOverTimeSwitch

'Add some colors to these ranges

ActiveSheet.Range("A1", Cells(TotalNumObjFn + 6, 1)).Select

With Selection.Interior

.ColorIndex $=39$

.Pattern $=$ xlSolid 


\section{End With}

'Create Worker Skill Matrix

For $\mathrm{i}=1$ To numworkers

For $\mathrm{k}=1$ To numskills

ActiveSheet.Cells(TotalNumObjFn $+8,1)$.Value = "Worker Skill Matrix"

ActiveSheet.Cells $(\mathrm{i}+$ TotalNumObjFn $+9,1)$.Value $=$ "Worker " \& i

If $\mathrm{k}<6$ Then ActiveSheet.Cells(TotalNumObjFn $+9, \mathrm{k}+1)$.Value $=$ "Skill " \& $\mathrm{k}$

If $\mathrm{k}>=6$ Then ActiveSheet.Cells(TotalNumObjFn $+9, \mathrm{k}+1$ ). Value $=\mathrm{k}$ Next k

Next i

'title coloring

ActiveSheet.Cells(TotalNumObjFn $+8,1$ ). Select

With Selection.Interior

.ColorIndex $=36$

.Pattern $=$ xlSolid

End With

'skill coloring

ActiveSheet.Range(Cells(TotalNumObjFn +9 , 2), Cells(TotalNumObjFn + 9, numskills + 1)).Select

With Selection.Interior

.ColorIndex $=40$

.Pattern $=$ xlSolid

End With

'worker coloring

ActiveSheet.Range(Cells(TotalNumObjFn + 10, 1), Cells(TotalNumObjFn + numworkers + 9, 1)).Select

With Selection.Interior

.ColorIndex $=34$

Pattern = xlSolid

End With 


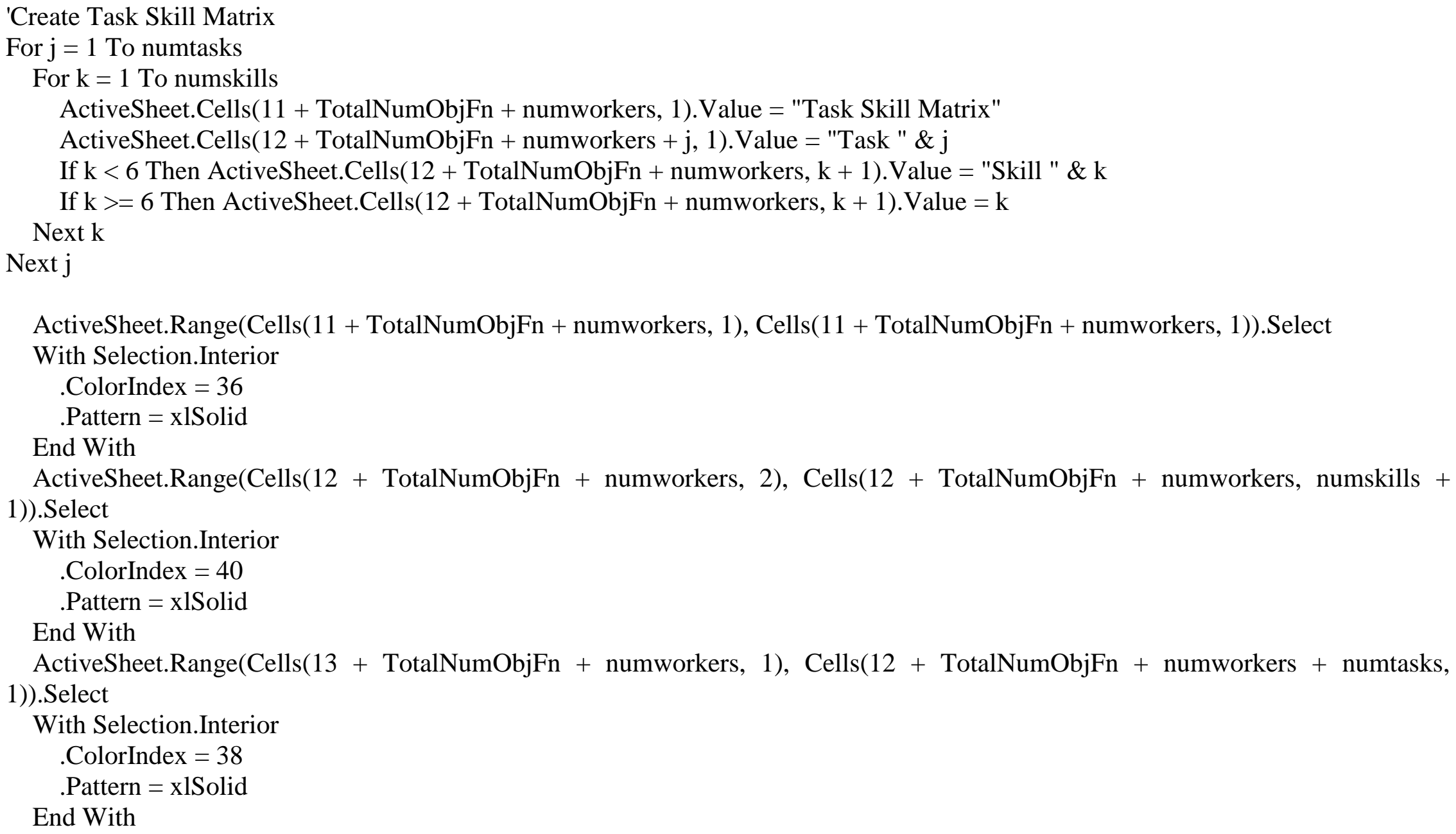


'Create Task Time Matrix

For $\mathrm{j}=1$ To numtasks

ActiveSheet.Cells $(14+$ TotalNumObjFn + numtasks + numworkers, 2).Value = "Task Time"

ActiveSheet.Cells $(14+$ TotalNumObjFn + numtasks + numworkers $+\mathrm{j}, 1)$. Value $=$ "Task " \& j Next j

ActiveSheet.Range(Cells $(14+$ TotalNumObjFn + numworkers + numtasks, 2$)$, Cells $(14+$ TotalNumObjFn + numworkers + numtasks, 2)).Select

With Selection.Interior

.ColorIndex $=36$

Pattern $=$ xlSolid

End With

ActiveSheet.Range(Cells $(15+$ TotalNumObjFn + numworkers + numtasks, 1$)$, Cells $(14+$ TotalNumObjFn + numworkers $+2 *$ numtasks, 1)).Select

With Selection.Interior

.ColorIndex $=38$

Pattern $=$ xlSolid

End With

'Create Worker Capacity Matrix

For $\mathrm{i}=1$ To numworkers

ActiveSheet.Cells $(16+$ TotalNumObjFn $+2 *$ numtasks + numworkers, 2).Value = "Worker Capacity"

ActiveSheet.Cells $(16+$ TotalNumObjFn $+2 *$ numtasks + numworkers $+\mathrm{i}, 1) \cdot$ Value $=$ "Worker " \& $\mathrm{i}$ Next i

ActiveSheet.Range(Cells(16 + TotalNumObjFn + numworkers $+2 *$ numtasks, 2$)$, Cells $(16+$ TotalNumObjFn + numworkers $+2 *$ numtasks, 3)).Select

With Selection.Interior

.ColorIndex $=36$

Pattern $=$ xlSolid 


\section{End With}

ActiveSheet.Range(Cells $(17+$ TotalNumObjFn + numworkers $+2 *$ numtasks, 1$)$, Cells $(16+$ TotalNumObjFn $+2 *$ numworkers + $2 *$ numtasks, 1)).Select

With Selection.Interior

.ColorIndex $=34$

Pattern $=$ xlSolid

End With

\section{'Create Training Cost Matrix}

For $\mathrm{i}=1$ To numskills

For $\mathrm{j}=1$ To 4

ActiveSheet.Cells $(18+$ TotalNumObjFn $+2 *$ numtasks $+2 *$ numworkers, 1$)$.Value $=$ "Cost to Train Matrix"

ActiveSheet.Cells $(19+$ TotalNumObjFn $+2 *$ numtasks $+2 *$ numworkers $+\mathrm{i}, 1)$.Value $=$ "Skill " \& i

ActiveSheet.Cells $(19+$ TotalNumObjFn $+2 *$ numtasks $+2 *$ numworkers, $1+\mathrm{j})$.Value $=$ "Level $" \& \mathrm{j} \& "$ to $" \& \mathrm{j}+1$

$$
\text { Next j }
$$

Next i

ActiveSheet.Range(Cells $(18+$ TotalNumObjFn $+2 *$ numworkers $+2 *$ numtasks, 1$)$, Cells $(18+$ TotalNumObjFn $+2 *$ numworkers $+2 *$ numtasks, 1$)$ ). Select

With Selection.Interior

.ColorIndex $=36$

.Pattern $=$ xlSolid

End With

ActiveSheet.Range(Cells $(20+$ TotalNumObjFn $+2 *$ numworkers $+2 *$ numtasks, 1$)$, Cells $(19+$ TotalNumObjFn $+2 *$ numworkers $+2 *$ numtasks + numskills, 1$)$ ). Select

With Selection.Interior

.ColorIndex $=40$

.Pattern $=$ xlSolid

End With 
ActiveSheet.Range(Cells(19+ TotalNumObjFn $+2 *$ numworkers $+2 *$ numtasks, 2$)$, Cells $(19+$ TotalNumObjFn $+2 *$ numworkers $+2 *$ numtasks, 5)).Select

With Selection.Interior

.ColorIndex $=35$

.Pattern $=$ xlSolid

End With

'Create Training Time Matrix

For $\mathrm{i}=1$ To numskills

For $\mathrm{j}=1$ To 4

ActiveSheet.Cells $(21+$ TotalNumObjFn $+2 *$ numtasks $+2 *$ numworkers + numskills, 1$)$. Value $=$ "Time to Train Matrix"

ActiveSheet.Cells $(22+$ TotalNumObjFn $+2 *$ numtasks $+2 *$ numworkers + numskills $+\mathrm{i}, 1)$.Value $=$ "Skill " \& i

$+1$

ActiveSheet.Cells $(22+$ TotalNumObjFn $+2 *$ numtasks $+2 *$ numworkers + numskills, $1+\mathrm{j})$. Value $=$ "Level " \& j \& " to " \& j Next j

Next $\mathrm{i}$

ActiveSheet.Range(Cells $(21+$ TotalNumObjFn $+2 *$ numworkers $+2 *$ numtasks + numskills, 1$)$, Cells $(21+$ TotalNumObjFn +2 $*$ numworkers $+2 *$ numtasks + numskills, 1)).Select

With Selection.Interior

.ColorIndex $=36$

.Pattern $=$ xlSolid

End With

ActiveSheet.Range(Cells $(23+$ TotalNumObjFn $+2 *$ numworkers $+2 *$ numtasks + numskills, 1$)$, Cells $(22+$ TotalNumObjFn +2

$*$ numworkers $+2 *$ numtasks $+2 *$ numskills, 1$)$ ). Select

With Selection.Interior

.ColorIndex $=40$

.Pattern $=$ xlSolid

End With 
ActiveSheet.Range(Cells $(22+$ TotalNumObjFn $+2 *$ numworkers $+2 *$ numtasks + numskills, 2$)$, Cells $(22+$ TotalNumObjFn +2 $*$ numworkers $+2 *$ numtasks + numskills, 5)).Select

With Selection.Interior

ColorIndex $=35$

.Pattern $=$ xlSolid

End With

'Create Worker Skill Preference Matrix

For $\mathrm{i}=1$ To numworkers

For $\mathrm{j}=1$ To numskills

Matrix"

ActiveSheet.Cells $(24+$ TotalNumObjFn $+2 *$ numtasks $+2 *$ numworkers $+2 *$ numskills, 1$)$. Value $=$ "Worker Preference

ActiveSheet.Cells $(25+$ TotalNumObjFn $+2 *$ numtasks $+2 *$ numworkers $+2 *$ numskills $+\mathrm{i}, 1)$.Value $=$ "Worker " \& i

$\& \mathrm{j}$

If $\mathrm{j}<6$ Then ActiveSheet.Cells $(25+$ TotalNumObjFn $+2 *$ numtasks $+2 *$ numworkers $+2 *$ numskills, $\mathrm{j}+1) . \mathrm{Value}=$ "Skill $"$

If $\mathrm{j}>=6$ Then ActiveSheet.Cells $(25+$ TotalNumObjFn $+2 *$ numtasks $+2 *$ numworkers $+2 *$ numskills, $\mathrm{j}+1) . \mathrm{Value}=\mathrm{j}$ Next $\mathrm{j}$

Next i

ActiveSheet.Range(Cells $(24+$ TotalNumObjFn $+2 *$ numworkers $+2 *$ numtasks $+2 *$ numskills, 1$)$, Cells $(24+$ TotalNumObjFn $+2 *$ numworkers $+2 *$ numtasks $+2 *$ numskills, 1$)$ ). Select

With Selection.Interior

.ColorIndex $=36$

.Pattern $=$ xlSolid

End With

ActiveSheet.Range(Cells $(26+$ TotalNumObjFn $+2 *$ numworkers $+2 *$ numtasks $+2 *$ numskills, 1$)$, Cells $(26+$ TotalNumObjFn $+3 *$ numworkers $+2 *$ numtasks $+2 *$ numskills $-1,1)$ ). Select

With Selection.Interior

.ColorIndex $=34$

.Pattern $=$ xlSolid 


\section{End With}

ActiveSheet.Range(Cells $(25+$ TotalNumObjFn $+2 *$ numworkers $+2 *$ numtasks $+2 *$ numskills, 2$)$, Cells $(25+$ TotalNumObjFn $+2 *$ numworkers $+2 *$ numtasks $+2 *$ numskills, numskills +1$)$ ). Select

With Selection.Interior

.ColorIndex $=40$

Pattern $=$ xlSolid

End With

End Sub

"There will be a sub similar to this one for each of the objectives.

"A separate procedure will simply call each of them needed

'This command button will find the initial solution for Minimum Training Cost

Public Sub InitalTrainingCostSoln_click()

Dim workerskill() As Single, oworkerskill() As Single

Dim taskskill() As Single, otaskskill() As Single

Dim tasktime() As Single, otasktime() As Single

Dim workercapacity() As Single, oworkercapacity() As Single

Dim traincost() As Single, otraincost() As Single

Dim traintime() As Single, otraintime() As Single

Dim workerassign() As Single

Dim workertaskcost() As Single, oworkertaskcost() As Single

Dim workertasktime() As Single, oworkertasktime() As Single

Dim workertaskSgap() As Single, oworkertaskSgap() As Single

Dim workertaskPref() As Single, oworkertaskPref() As Single

Dim Prefmatrix() As Single

Dim taskassigned() As Single 
Dim tcost() As Single

Dim ttime() As Single

Dim available() As Single

Dim bestworkerassign() As Single

Dim numworkers As Single, numskills As Single, numtasks As Single, numSolns As Single

Dim totaltaskcost() As Single

Dim totalworkercost() As Single

Dim totaltaskSgap() As Single

Dim totalworkerSgap() As Single

Dim totaltaskPref() As Single

Dim totalworkerPref() As Single

Dim workerphase1() As Single

Dim cellrow As Single

Dim trainingneeds() As Single

Dim skilltrainingneeds() As Single

'everything below is new:

Dim bestCost() As Single

Dim bestSgap() As Single

Dim bestPref() As Single

Dim TotalNumObjFn As Single

TotalNumObjFn $=3$

Sheets("Results Summary").Cells.Clear

Sheets("Parameters").Select

p1perprior $=$ ActiveSheet.Cells $(1,2)$.Value

p1 perrestrict $=$ ActiveSheet.Cells $(2,2)$. Value 
perprior $=$ ActiveSheet.Cells (3, 2).Value

perrestrict $=$ ActiveSheet.Cells $(4,2)$.Value

numiter $=$ ActiveSheet.Cells $(5,2)$.Value

phase1_on = 1 'this can be used as a switch to turn phase 1 on or off

Sheets("Input").Select

numworkers $=$ ActiveSheet.Cells(2, 2). Value

numskills $=$ ActiveSheet.Cells $(3,2)$. Value

numtasks $=$ ActiveSheet.Cells $(4,2)$.Value

numSolns = ActiveSheet.Cells $(5,2)$.Value

'initialize arrays

ReDim workerskill(0 To numworkers + 1, 0 To numskills + 1) As Single

ReDim oworkerskill( 0 To numworkers $+1,0$ To numskills +1$)$ As Single

ReDim taskskill( 0 To numtasks $+1,0$ To numskills +1$)$ As Single

ReDim otaskskill(0 To numtasks $+1,0$ To numskills +1$)$ As Single

ReDim tasktime (0 To numtasks + 1) As Single

ReDim otasktime (0 To numtasks +1$)$ As Single

ReDim workercapacity $(0$ To numworkers +1$)$ As Single

ReDim oworkercapacity (0 To numworkers +1$)$ As Single

ReDim traincost(0 To numskills $+1,0$ To 5, 0 To 5) As Single

ReDim otraincost $(0$ To numskills $+1,0$ To 5, 0 To 5) As Single

ReDim traintime(0 To numskills $+1,0$ To 5, 0 To 5) As Single

ReDim otraintime( 0 To numskills $+1,0$ To 5, 0 To 5) As Single

'added a dimension to the two matrices below

ReDim workerassign( $(0$ To 1,0 To numworkers $+1,0$ To numtasks +1$)$ As Single

ReDim bestworkerassign $(0$ To numSolns $+1,0$ To numworkers $+1,0$ To numtasks +1 ) As Single

ReDim workertaskcost $(0$ To numworkers $+1,0$ To numtasks +1$)$ As Single

ReDim oworkertaskcost $(0$ To numworkers $+1,0$ To numtasks +1$)$ As Single 
ReDim workertaskSgap $(0$ To numworkers $+1,0$ To numtasks +1$)$ As Single ReDim oworkertaskSgap (0 To numworkers $+1,0$ To numtasks +1$)$ As Single ReDim workertaskPref $(0$ To numworkers $+1,0$ To numtasks +1$)$ As Single

ReDim oworkertaskPref $(0$ To numworkers $+1,0$ To numtasks +1$)$ As Single ReDim Prefmatrix $(0$ To numworkers $+1,0$ To numskills +1$)$ As Single

ReDim workertasktime $(0$ To numworkers $+1,0$ To numtasks +1$)$ As Single ReDim oworkertasktime $(0$ To numworkers $+1,0$ To numtasks +1$)$ As Single ReDim taskassigned(0 To numtasks +1 ) As Single ReDim tcost $(0$ To numskills $+1,0$ To 5) As Single ReDim ttime(0 To numskills $+1,0$ To 5) As Single

ReDim available $(0$ To numworkers $*$ numtasks $+1,0$ To 3 ) As Single

ReDim totaltaskcost $(0$ To numtasks +1$)$ As Single

ReDim totalworkercost(0 To numworkers + 1) As Single

ReDim totaltaskSgap $(0$ To numtasks +1$)$ As Single

ReDim totalworkerSgap (0 To numworkers +1$)$ As Single

ReDim totaltaskPref(0 To numtasks +1$)$ As Single

ReDim totalworkerPref $(0$ To numworkers +1$)$ As Single

ReDim workerphase1(0 To numworkers +1$)$ As Single

ReDim trainingneeds $(0$ To numworkers $+1,0$ To numskills +1$)$ As Single

ReDim skilltrainingneeds $(0$ To numskills $+1,0$ To 5) As Single

'new one below:

ReDim bestCost $(0$ To numSolns + 20) As Single

ReDim bestSgap(0 To numSolns + 20) As Single

ReDim bestPref(0 To numSolns + 20) As Single

starttime $=$ Timer

For $\mathrm{a}=0$ To numSolns +1

bestCost $(a)=0$

bestSgap $(a)=0$

bestPref(a) $=0$ 
For $\mathrm{b}=0$ To numworkers +1

For $\mathrm{c}=0$ To numtasks +1

bestworkerassign $(a, b, c)=0$

Next c

Next b

Next a

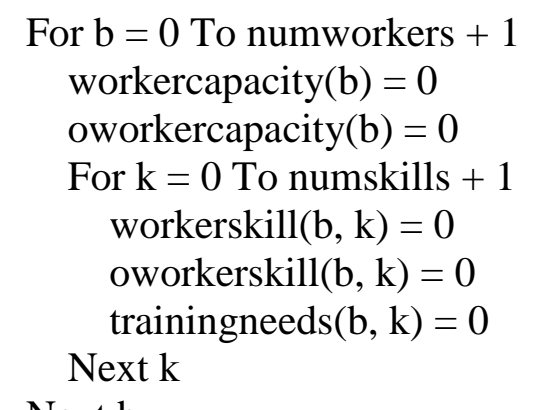

Next b

For $\mathrm{b}=0$ To numworkers $*$ numtasks +1

For $\mathrm{k}=0$ To 3

available $(\mathrm{b}, \mathrm{k})=0$

Next k

Next b

For $\mathrm{b}=0$ To numtasks +1

tasktime $(b)=0$

otasktime $(\mathrm{b})=0$

taskassigned(b) $=0$

totaltaskcost $(b)=0$

totaltaskSgap $(b)=0$ 


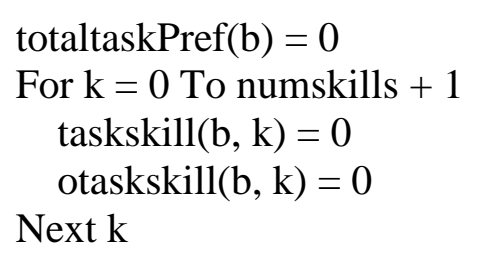

For $b=1$ To numworkers

totalworkercost $(b)=0$

totalworkerSgap $(\mathrm{b})=0$

totalworkerPref $(b)=0$

For $\mathrm{k}=1$ To numtasks workerassign $(1, \mathrm{~b}, \mathrm{k})=0$ workertaskcost $(\mathrm{b}, \mathrm{k})=0$ $\operatorname{oworkertask\operatorname {cost}}(\mathrm{b}, \mathrm{k})=0$ workertaskSgap $(b, k)=0$ $\operatorname{oworkertaskSgap}(\mathrm{b}, \mathrm{k})=0$ 
workertaskPref $(\mathrm{b}, \mathrm{k})=0$

oworkertaskPref(b, k) $=0$

Next k

Next b

'read in data from file

For $\mathrm{b}=1$ To numworkers

For $\mathrm{k}=1$ To numskills

oworkerskill $(\mathrm{b}, \mathrm{k})=$ ActiveSheet.Cells $(9+$ TotalNumObjFn $+\mathrm{b}, 1+\mathrm{k})$

Next k

Next b

For $\mathrm{b}=1$ To numtasks

For $\mathrm{k}=1$ To numskills

otaskskill $(\mathrm{b}, \mathrm{k})=$ ActiveSheet.Cells $(9+$ TotalNumObjFn + numworkers $+3+\mathrm{b}, 1+\mathrm{k})$

Next k

Next b

For $\mathrm{b}=1$ To numtasks

otasktime $(\mathrm{b})=$ ActiveSheet.Cells $(9+$ TotalNumObjFn + numworkers $+3+$ numtasks $+2+b, 2)$

Next b

For $b=1$ To numworkers

oworkercapacity $(b)=$ ActiveSheet.Cells $(9+$ TotalNumObjFn + numworkers $+3+$ numtasks $+2+$ numtasks $+2+b, 2)$ Next b

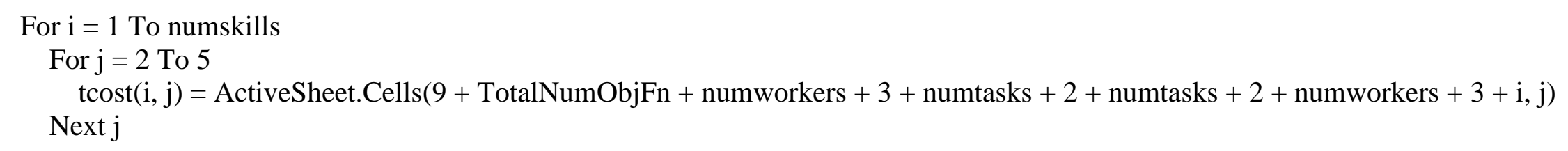


Next i

For $\mathrm{i}=1$ To numskills

For $\mathrm{j}=2$ To 5

ttime $(\mathrm{i}, \mathrm{j})=$ ActiveSheet.Cells $(9+$ TotalNumObjFn + numworkers $+3+$ numtasks $+2+$ numtasks $+2+$ numworkers $+3+$ numskills $+3+\mathrm{i}, \mathrm{j}$ )

Next j

Next i

For $b=1$ To numworkers

For $\mathrm{k}=1$ To numskills

Prefmatrix $(b, k)=$ ActiveSheet.Cells $(9+$ TotalNumObjFn + numworkers $+3+$ numtasks $+2+$ numtasks $+2+$ numworkers +3 + numskills $+3+$ numskills $+3+\mathrm{b}, \mathrm{k}+1)$ Next k

Next b

'done reading in values from the excel sheet

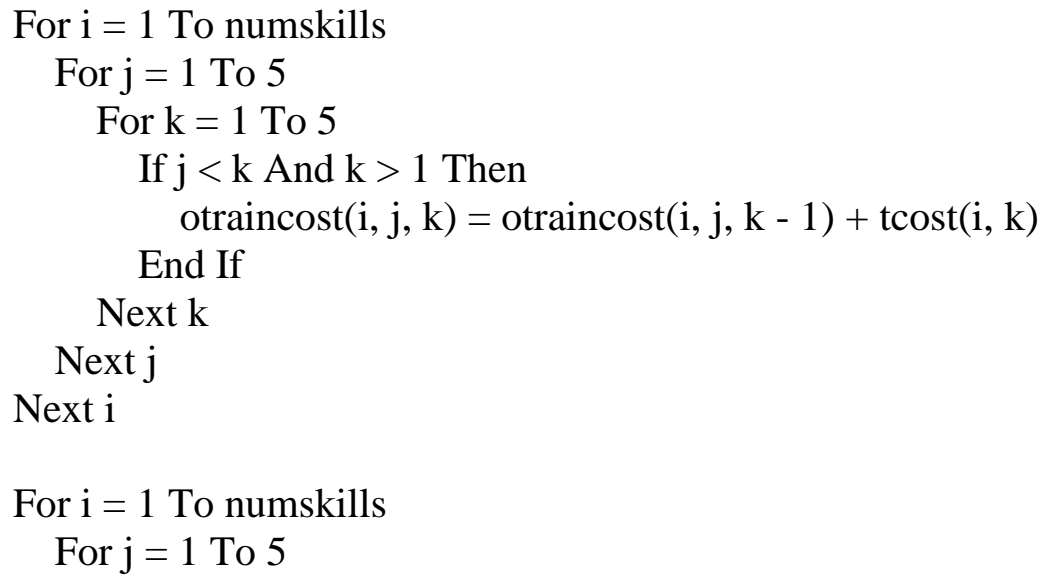




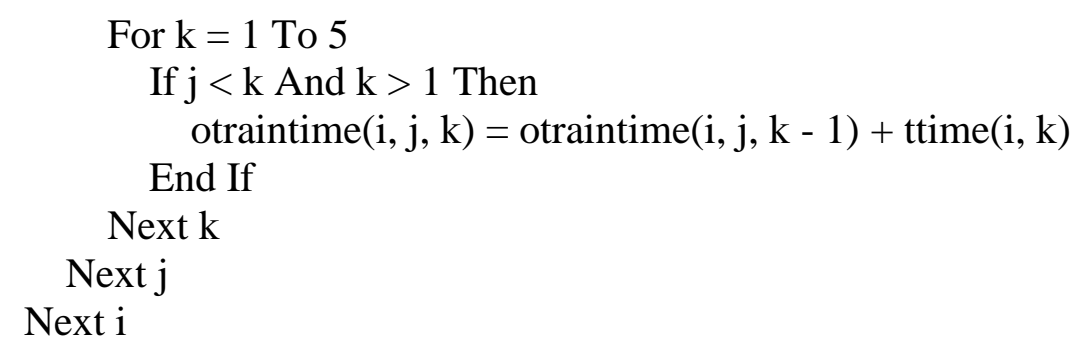

'find task cost and training time for each worker for each task

For $\mathrm{i}=1$ To numworkers

For $\mathrm{j}=1$ To numtasks

oworkertasktime $(\mathrm{i}, \mathrm{j})=\operatorname{otasktime}(\mathrm{j})$

For $\mathrm{k}=1$ To numskills

If oworkerskill(i, k) < otaskskill(j, k) And otaskskill(j, k) > 1 Then

$\operatorname{oworkertaskcost}(i, j)=\operatorname{oworkertaskcost}(i, j)+\operatorname{otraincost}(k, \operatorname{oworkerskill}(i, k)$, otaskskill $(j, k))$

$\operatorname{oworkertaskSgap}(i, j)=\operatorname{oworkertaskSgap}(i, j)+(\operatorname{otaskskill}(j, k)-\operatorname{oworkerskill}(i, k))$

$\operatorname{oworkertaskPref}(i, j)=\operatorname{oworkertaskPref}(i, j)+\operatorname{Prefmatrix}(i, k) *(\operatorname{otaskskill}(j, k)-\operatorname{oworkerskill}(i, k))$

oworkertasktime $(i, j)=$ oworkertasktime $(i, j)+\operatorname{otraintime}(k$, oworkerskill( $(i, k)$, otaskskill $(j, k))$

End If

Next $k$

Next j

Next i

For $\mathrm{j}=1$ To numtasks

For $\mathrm{i}=1$ To numworkers

totaltaskcost $(j)=$ totaltaskcost $(j)+$ oworkertaskcost $(i, j)$

totaltaskSgap $(\mathrm{j})=$ totaltaskSgap $(\mathrm{j})+$ oworkertaskSgap $(\mathrm{i}, \mathrm{j})$

totaltaskPref $(\mathrm{j})=$ totaltaskPref $(\mathrm{j})+\operatorname{oworkertaskPref}(\mathrm{i}, \mathrm{j})$

Next $i$

Next j 


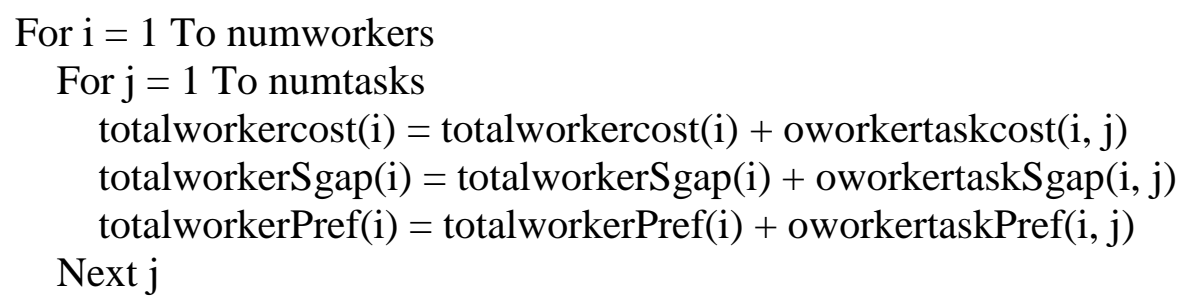

\section{For $\mathrm{a}=1$ To numSolns +1}

bestCost $(\mathrm{a})=9999999$

bestSgap (a) $=-9999999$

bestPref(a) = -9999999

\section{Next a}

bestCost $(0)=-9999999$

bestSgap $(0)=9999999$

bestPref $(0)=9999999$

For $r=1$ To numiter

'copy original data into matrices

For $b=1$ To numworkers

workercapacity $(b)=$ oworkercapacity $(b)$

For $\mathrm{k}=1$ To numskills

workerskill $(b, k)=\operatorname{oworkerskill}(b, k)$ 


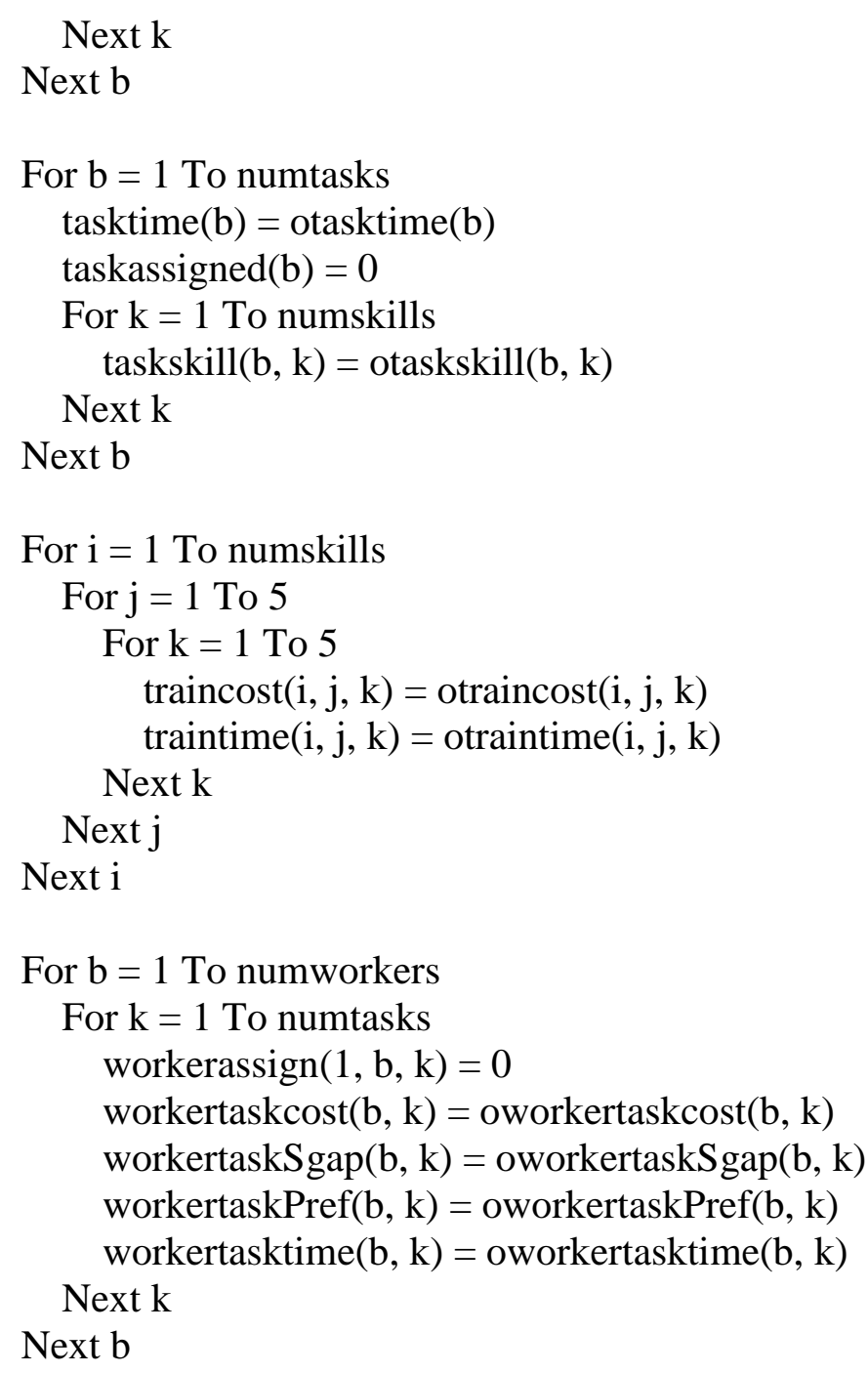

For $\mathrm{b}=1$ To numworkers 


$$
\text { workerphase } 1(b)=0
$$

\section{Next b}

totalcost $=0$

totalsgap $=0$

totalpref $=0$

numtaskassigned $=0$

If phase1_on = 1 Then 'this can be used as a switch to turn phase 1 on or off

'start phase 1 - each worker assigned 1 task

Do While numtaskassigned < numworkers

'find lowest skilled worker - worker with the highest totalcost

'make sure they are not already assigned

$\operatorname{maxcost}=-55$

For $\mathrm{i}=1$ To numworkers

If workerphase $1(i)=0$ And totalworkercost $(i)>\operatorname{maxcost}$ Then

maxcost $=$ totalworkercost $(\mathrm{i})$

maxcostworker $=\mathrm{i}$

$$
\text { End If }
$$

Next i

'find lowest cost task for maxcost worker - make sure task not already assigned

'make sure worker has enough capacity

mincost $=99999999$

mincosttask $=0$

For $\mathrm{j}=1$ To numtasks

If taskassigned(j) = 0 And workertasktime(maxcostworker, j) $<=$ workercapacity(maxcostworker) And workertaskcost (maxcostworker, $\mathrm{j})<$ mincost Then

mincost $=$ workertaskcost $($ maxcostworker, $\mathrm{j})$ 
mincosttask $=\mathrm{j}$

End If

Next j

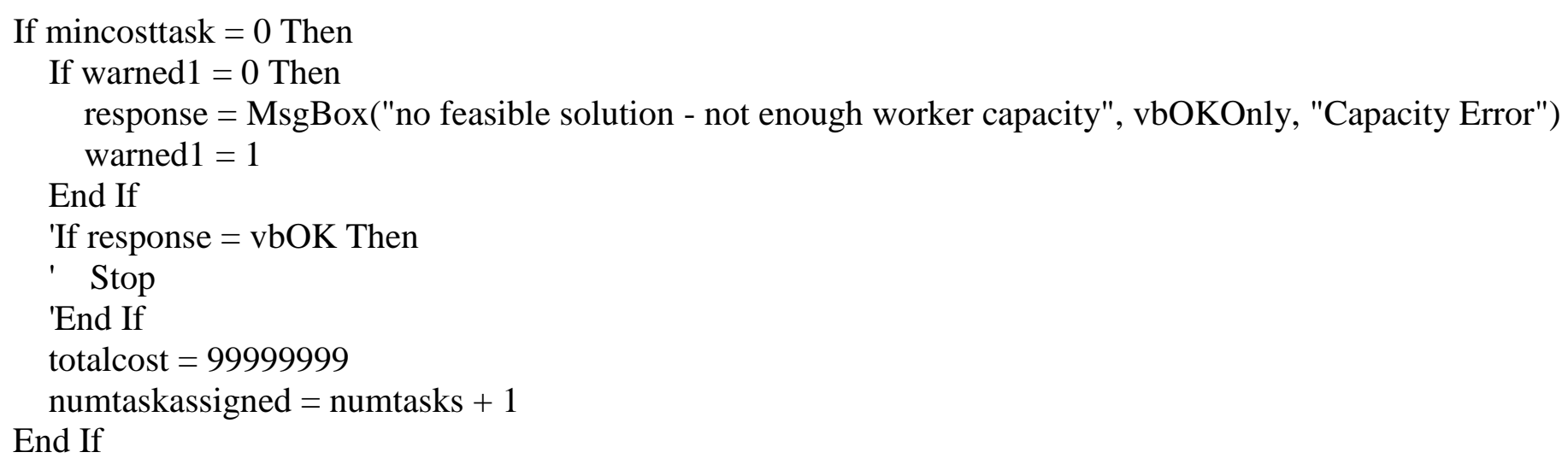

\section{Randomize}

priorrnd $=\operatorname{Round}(((100-1) *$ Rnd $)+1)$

'make the best assignment

If priorrnd $<=$ p1 perprior Then

'assign maxcostworker to mincost task

totalcost $=$ totalcost + workertaskcost $($ maxcostworker, mincosttask $)$

totalsgap $=$ totalsgap + workertaskSgap $($ maxcostworker, mincosttask $)$

totalpref $=$ totalpref + workertaskPref $($ maxcostworker, mincosttask $)$

numtaskassigned $=$ numtaskassigned +1

workerassign $(1$, maxcostworker, mincosttask $)=1$

taskassigned $(\operatorname{mincosttask})=1$

workerphase $1($ maxcostworker $)=1$ 
workercapacity $($ maxcostworker $)=$ workercapacity $($ maxcostworker $)$ - workertasktime $($ maxcostworker, mincosttask $)$ assignedworker $=$ maxcostworker

assignedtask $=$ mincosttask

\section{End If}

'make a near-optimal assignment

If priorrnd > p1perprior Then

'form available list and choose assigned worker from available list

numonlist $=0$

For $\mathrm{i}=1$ To numworkers

If workerphase1(i) $=0$ And totalworkercost $(\mathrm{i})>=\max \operatorname{cost} *(1-(\mathrm{p} 1$ perrestrict $/ 100))$ Then For $\mathrm{j}=1$ To numtasks

If taskassigned $(\mathrm{j})=0$ And workertaskcost $(\mathrm{i}, \mathrm{j})<=\operatorname{mincost} *(1+(\mathrm{p} 1$ perrestrict $/ 100))$ And workertasktime $(\mathrm{i}, \mathrm{j})$ $<=$ workercapacity(i) Then
numonlist $=$ numonlist +1
available(numonlist, 1$)=\mathrm{i}$
available(numonlist, 2$)=\mathrm{j}$

\section{End If}

Next $j$

End If

Next i

'determine random assignment from the candidate list created above

Randomize

restrictrnd $=\operatorname{Round}((($ numonlist -1$) *$ Rnd $)+1)$

assignedworker $=$ available $($ restrictrnd, 1$)$

assignedtask $=$ available $($ restrictrnd, 2$)$

'increase each objective function

totalcost $=$ totalcost + workertaskcost(assignedworker, assignedtask)

totalsgap $=$ totalsgap + workertaskSgap(assignedworker, assignedtask) 
totalpref $=$ totalpref + workertaskPref(assignedworker, assignedtask $)$

'adjust counter variables

numtaskassigned $=$ numtaskassigned +1

$\operatorname{workerassign}(1$, assignedworker, assignedtask $)=1$

taskassigned (assignedtask $)=1$

workerphase 1 (assignedworker $)=1$

'reduce assigned workers capacity

workercapacity (assignedworker) = workercapacity(assignedworker) - workertasktime(assignedworker, assignedtask) End If

'update workerskills for assignedworker based on training received for assignedtask

For $\mathrm{k}=1$ To numskills

If workerskill(assignedworker, $\mathrm{k}$ ) < taskskill(assignedtask, $\mathrm{k}$ ) Then

workerskill(assignedworker, $\mathrm{k}$ ) = taskskill(assignedtask, $\mathrm{k}$ )

End If

Next k

'update workertaskcost and workertasktime for assignedworker

For $\mathrm{j}=1$ To numtasks

If taskassigned $(j)=0$ Then

'zero out all the objective function values and recalculate them with new skill levels determined above

workertaskcost (assignedworker, $\mathrm{j})=0$

workertaskSgap (assignedworker, $\mathrm{j})=0$

workertaskPref(assignedworker, $\mathrm{j}$ ) $=0$

workertasktime (assignedworker, $\mathrm{j})=$ otasktime $(\mathrm{j})$

For $\mathrm{k}=1$ To numskills

If workerskill(assignedworker, $\mathrm{k}$ ) < taskskill(j, $\mathrm{k}$ ) And taskskill( $\mathrm{j}, \mathrm{k})>1$ Then

k), taskskill(j, k))

workertaskcost $($ assignedworker, $\mathrm{j})=$ workertaskcost $($ assignedworker, $\mathrm{j})+\operatorname{traincost}(\mathrm{k}$, workerskill(assignedworker, 


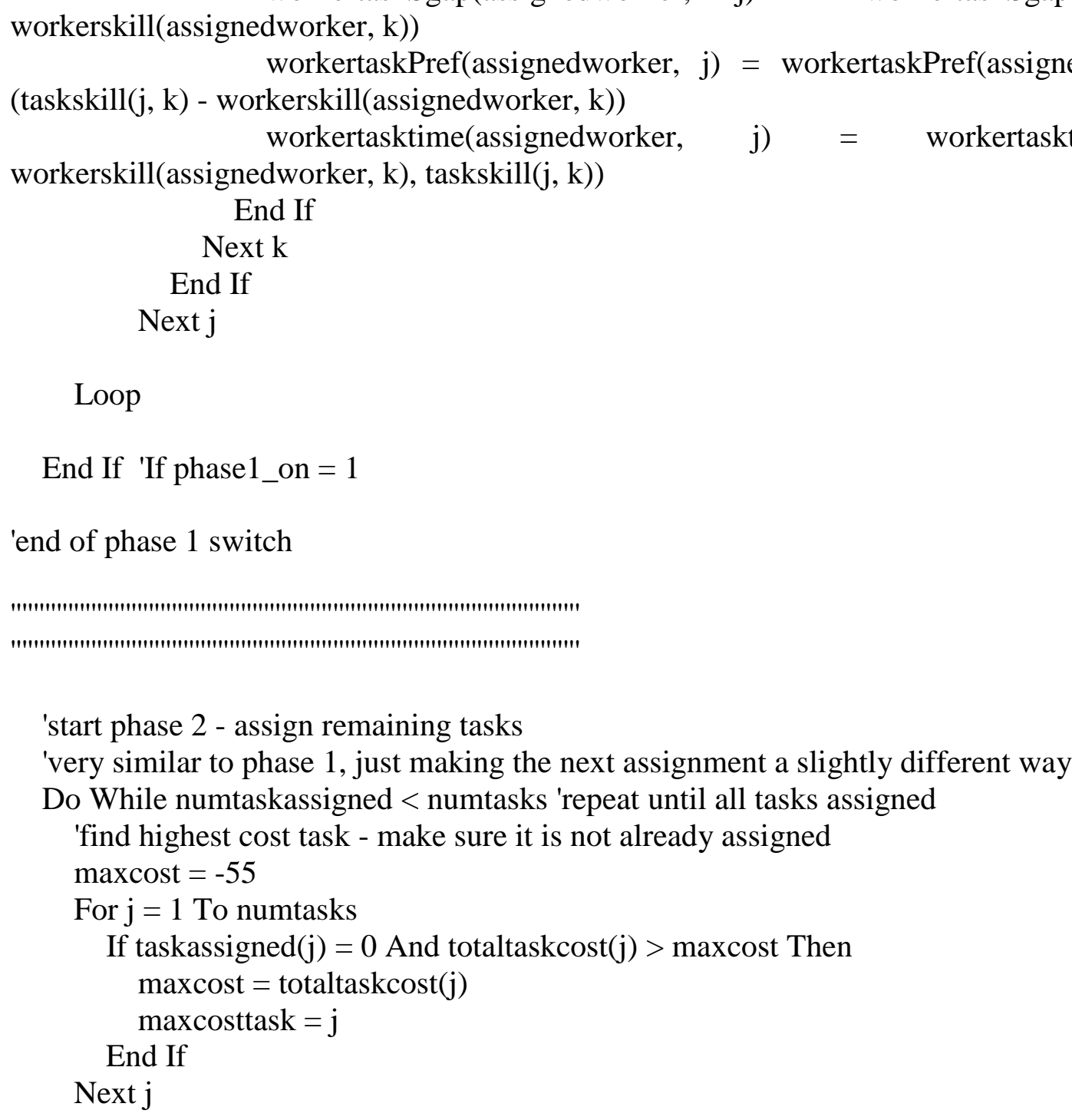

End If 'If phase1_on $=1$

'end of phase 1 switch

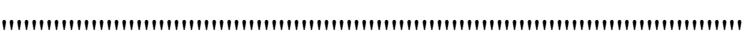

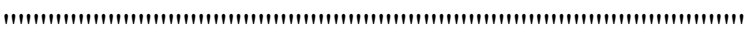

'start phase 2 - assign remaining tasks

'very similar to phase 1 , just making the next assignment a slightly different way

Do While numtaskassigned < numtasks 'repeat until all tasks assigned

'find highest cost task - make sure it is not already assigned

maxcost $=-55$

For $\mathrm{j}=1$ To numtasks

If taskassigned $(j)=0$ And totaltaskcost $(j)>\operatorname{maxcost}$ Then

$\operatorname{maxcost}=$ totaltaskcost $(\mathrm{j})$

$\operatorname{maxcosttask}=\mathrm{j}$

\section{End If}

Next j 
'find lowest cost worker for highest cost task - make sure worker has enough capacity

mincost $=9999999$

mincostworker $=0$

For $\mathrm{i}=1$ To numworkers

If workertasktime(i, maxcosttask) <= workercapacity(i) And workertaskcost(i, maxcosttask) < mincost Then mincost $=$ workertaskcost $(i$, maxcosttask $)$

mincostworker $=\mathrm{i}$

End If

Next i

Randomize

priorrnd $=\operatorname{Round}(((100-1) *$ Rnd $)+1)$

If mincostworker $>0$ Then

If priorrnd $<=$ perprior Then

'assign mincostworker to maxcost task; update objective function values

totalcost $=$ totalcost + workertaskcost $($ mincostworker, maxcosttask $)$

totalsgap $=$ totalsgap + workertaskSgap $($ mincostworker, maxcosttask $)$

totalpref $=$ totalpref + workertaskPref(mincostworker, maxcosttask)

'update counter variables

numtaskassigned $=$ numtaskassigned +1

workerassign $(1$, mincostworker, maxcosttask $)=1$

taskassigned $(\operatorname{maxcosttask})=1$

workercapacity $($ mincostworker $)=$ workercapacity $($ mincostworker $)$ - workertasktime $($ mincostworker, maxcosttask $)$

assigned worker $=$ mincostworker

assignedtask $=$ maxcosttask

End If

If priorrnd > perprior Then 
'form available list and choose assigned worker from available list numonlist $=0$

For $\mathrm{j}=1$ To numtasks

If totaltaskcost $(\mathrm{j})>=$ maxcost $*(1-($ perrestrict $/ 100))$ And taskassigned $(\mathrm{j})=0$ Then

For $\mathrm{i}=1$ To numworkers

If workertaskcost $(\mathrm{i}, \mathrm{j})<=$ mincost $*(1+$ (perrestrict / 100)) And workertasktime $(\mathrm{i}, \mathrm{j})<=$ workercapacity(i) Then numonlist $=$ numonlist +1

available(numonlist, 1$)=\mathrm{i}$

available(numonlist, 2 ) $=\mathrm{j}$

End If

Next i

End If

Next j

Randomize

restrictrnd $=\operatorname{Round}((($ numonlist -1$) *$ Rnd $)+1)$

assignedworker $=$ available $($ restrictrnd, 1$)$

assignedtask $=$ available $($ restrictrnd, 2$)$

totalcost $=$ totalcost + workertaskcost(assignedworker, assignedtask)

totalsgap $=$ totalsgap + workertaskSgap(assignedworker, assignedtask)

totalpref $=$ totalpref + workertaskPref(assignedworker, assignedtask)

numtaskassigned $=$ numtaskassigned +1

workerassign $(1$, assignedworker, assignedtask $)=1$

taskassigned (assignedtask $)=1$

workercapacity (assignedworker) = workercapacity (assignedworker) - workertasktime (assignedworker, assignedtask)

End If

'update workerskills for assignedworker based on training received for assignedtask

For $\mathrm{k}=1$ To numskills 
If workerskill(assignedworker, $\mathrm{k}$ ) < taskskill(assignedtask, $\mathrm{k}$ ) Then

workerskill(assignedworker, $\mathrm{k}$ ) = taskskill(assignedtask, $\mathrm{k}$ )

\section{End If}

Next k

'update workertaskcost and workertasktime for assignedworker

For $\mathrm{j}=1$ To numtasks

If taskassigned $(j)=0$ Then

workertaskcost(assignedworker, $\mathrm{j})=0$

workertaskSgap(assignedworker, $\mathrm{j}$ ) $=0$

workertaskPref(assignedworker, $\mathrm{j}$ ) $=0$

workertasktime (assignedworker, $\mathrm{j}$ ) = otasktime $(\mathrm{j})$

For $\mathrm{k}=1$ To numskills

If workerskill(assignedworker, $\mathrm{k})<\operatorname{taskskill(j,k)}$ And taskskill(j, $\mathrm{k})>1$ Then

$\mathrm{k})$, taskskill(j, k))

workertaskcost $($ assignedworker, $\mathrm{j})=$ workertaskcost $(\operatorname{assignedworker,~} \mathrm{j})+\operatorname{traincost}(\mathrm{k}$, workerskill(assignedworker,

workertaskSgap(assignedworker, j) = workertaskSgap(assignedworker, j) $\quad+\quad(\operatorname{taskskill}(\mathrm{j}, \quad \mathrm{k}) \quad-$ workerskill(assignedworker, $\mathrm{k})$ )

workertaskPref(assignedworker, j) = workertaskPref(assignedworker, j) + Prefmatrix(assignedworker, k) * (taskskill(j, k) - workerskill(assignedworker, k))

k), taskskill $(\mathrm{j}, \mathrm{k})$ )

workertasktime $($ assignedworker, $\mathrm{j})=$ workertasktime $($ assignedworker, $\mathrm{j})+\operatorname{traintime}(\mathrm{k}$, workerskill(assignedworker,

$$
\begin{aligned}
& \text { End If } \\
& \text { Next k } \\
& \text { End If } \\
& \text { Next j }
\end{aligned}
$$

End If

If mincostworker $=0$ Then 


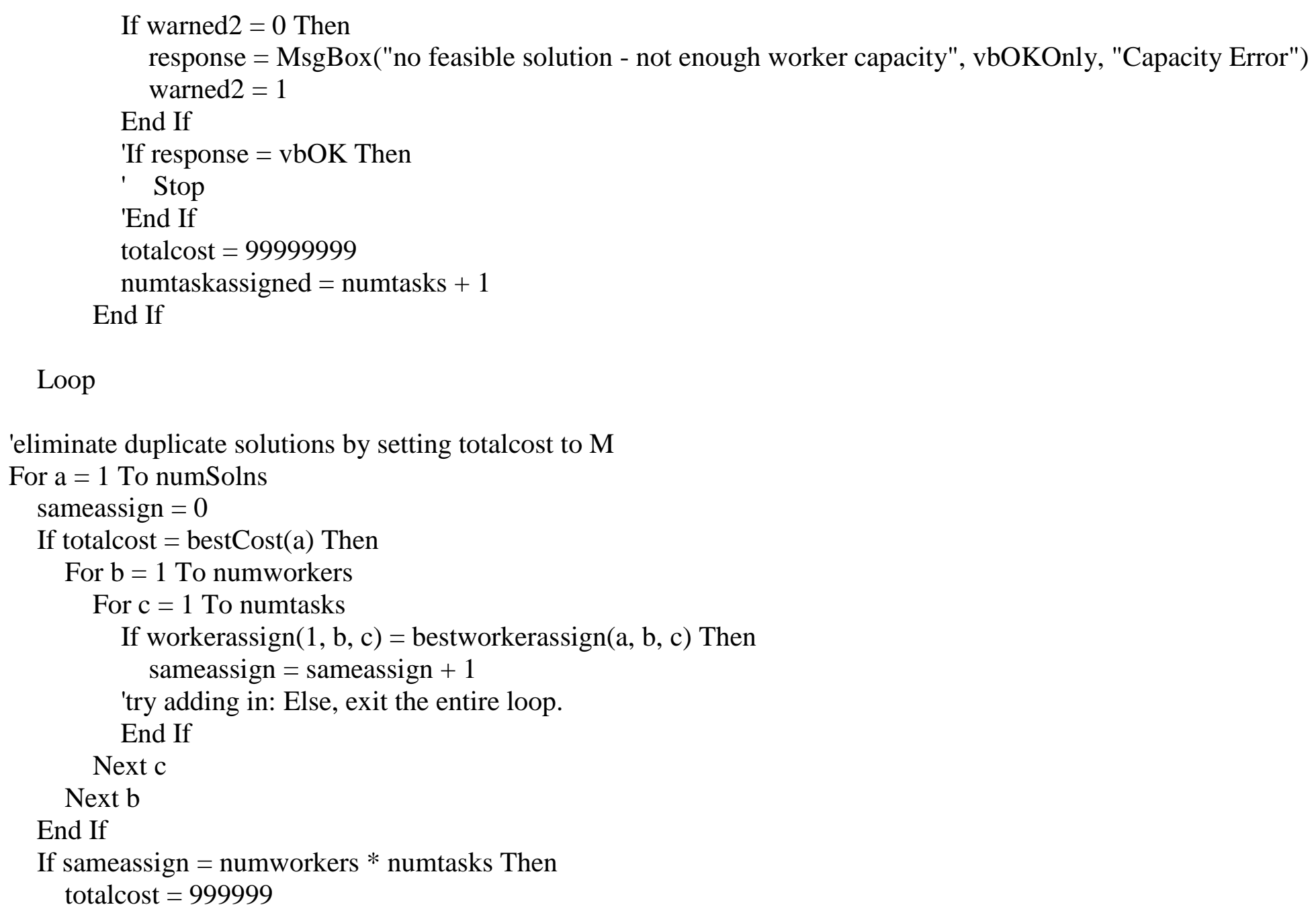


'check to see if totalcost is one of the top solutions. if so, everything behind it gets bumped 'and totalcost replaces the top solution that it beats out (a determines this)

If totalcost $<=$ bestCost(numSolns) Then

For a $=$ numSolns To 1 Step -1

'determines where in the list the new solution goes

If totalcost $<=$ bestCost(a) And totalcost $>$ bestCost $(a-1)$ Then

$\mathrm{b}=$ numSolns

$\mathrm{c}=\mathrm{a}$

'move the lower solutions back one rank

Do While $\mathrm{c}<$ numSolns

bestCost $(b)=\operatorname{bestCost}(b-1)$

$\operatorname{bestSgap}(b)=$ bestSgap $(b-1)$

bestPref $(b)=\operatorname{bestPref}(b-1)$

For $\mathrm{i}=1$ To numworkers

For $\mathrm{j}=1$ To numtasks

$\operatorname{bestworkerassign}(b, i, j)=\operatorname{bestworkerassign}(b-1, i, j)$

Next $j$

\section{Next i}

$\mathrm{c}=\mathrm{c}+1$

$\mathrm{b}=\mathrm{b}-1$

\section{Loop}

'set the new solution into its rank

bestCost $(\mathrm{a})=$ totalcost

bestSgap $(\mathrm{a})=$ totalsgap

bestPref $(a)=$ totalpref

For $\mathrm{i}=1$ To numworkers

For $\mathrm{j}=1$ To numtasks

bestworkerassign $(a, i, j)=\operatorname{workerassign}(1, i, j)$

$$
\text { Next j }
$$

Next i

End If 
Next a

End If

'Print results

Sheets("Results Summary").Select

ActiveSheet.Cells $(1,1)=$ "Best Solution Costs"

ActiveSheet.Cells $(2,1)=$ "Training Amount"

ActiveSheet.Cells $(3,1)=$ "Worker Preference"

For $\mathrm{a}=1$ To numSolns

ActiveSheet.Cells $(1, a+1)=\operatorname{bestCost}(a)$

Next a

For $\mathrm{a}=1$ To numSolns

ActiveSheet.Cells $(2, a+1)=\operatorname{bestSgap}(a)$

Next a

For $\mathrm{a}=1$ To numSolns

ActiveSheet.Cells $(3, a+1)=\operatorname{bestPref}(a)$

Next a

Next $r$

endtime $=$ Timer

totaltime $=$ endtime - starttime

Sheets("Results Summary").Select

ActiveSheet.Cells $(1$, numSolns +3$)=$ "Run Time"

ActiveSheet.Cells $(1$, numSolns +4$)=$ totaltime

'Print to Results Summary sheet

Sheets("Results Summary").Select

'ActiveSheet.Cells $(1,1)=$ "Best Solution Costs"

'ActiveSheet.Cells $(1,2)=$ bestsolution(1) 
ActiveSheet.Cells $(6,1)$ = "Solution \#:"

For $\mathrm{a}=1$ To numSolns

ActiveSheet.Cells $(6,2 * a)=a$

Next a

ActiveSheet.Cells $(5,1)=$ "Worker to Task Assignments" ActiveSheet.Cells $(8+$ numtasks, 1$)=$ "Total Cost"

ActiveSheet.Cells $(9+$ numtasks, 1$)=$ "Total Skill Levels" ActiveSheet.Cells $(10+$ numtasks, 1$)=$ "Total Preference" $\mathrm{b}=1$

For $\mathrm{a}=1$ To numSolns $* 2$

ActiveSheet.Cells(7, a) = "Worker"

ActiveSheet.Cells $(7, a+1)=$ "Task"

cellrow $=8$

For $\mathrm{i}=1$ To numworkers

For $\mathrm{j}=1$ To numtasks

If bestworkerassign $(b, i, j)=1$ Then

ActiveSheet.Cells(cellrow, a) $=\mathrm{i}$

ActiveSheet.Cells (cellrow, $a+1)=j$

cellrow $=$ cellrow +1

\section{End If}

Next $j$

\section{Next i}

ActiveSheet.Cells $(8+$ numtasks, $a+1)=\operatorname{bestCost}(b)$

ActiveSheet.Cells $(9+$ numtasks, $a+1)=$ bestSgap $(b)$

ActiveSheet.Cells $(10+$ numtasks, $a+1)=$ bestPref(b)

$\mathrm{b}=\mathrm{b}+1$

$\mathrm{a}=\mathrm{a}+1$

Next a

ActiveSheet.Range("A1").Select 


\author{
With Selection.Interior \\ .ColorIndex $=35$ \\ .Pattern $=$ xlSolid \\ End With \\ ActiveSheet.Range("A4:B4").Select \\ With Selection.Interior \\ .ColorIndex $=36$ \\ .Pattern $=$ xlSolid \\ End With
}


Public Sub InitialSkillGapSoln_click()

Dim workerskill() As Single, oworkerskill() As Single

Dim taskskill() As Single, otaskskill() As Single

Dim tasktime() As Single, otasktime() As Single

Dim workercapacity() As Single, oworkercapacity() As Single

Dim traincost() As Single, otraincost() As Single

Dim traintime() As Single, otraintime() As Single

Dim workerassign() As Single

Dim workertaskcost() As Single, oworkertaskcost() As Single

Dim workertasktime() As Single, oworkertasktime() As Single

Dim workertaskSgap() As Single, oworkertaskSgap() As Single

Dim workertaskPref() As Single, oworkertaskPref() As Single

Dim Prefmatrix() As Single

Dim taskassigned() As Single

Dim tcost() As Single

Dim ttime() As Single

Dim available() As Single

Dim bestworkerassign() As Single

Dim numworkers As Single, numskills As Single, numtasks As Single, numSolns As Single

Dim totaltaskcost() As Single

Dim totalworkercost() As Single

Dim totaltaskSgap() As Single

Dim totalworkerSgap() As Single

Dim totaltaskPref() As Single

Dim totalworkerPref() As Single

Dim workerphase1() As Single

Dim cellrow As Single

Dim trainingneeds() As Single

Dim skilltrainingneeds() As Single

Dim bestCost() As Single 
Dim bestSgap() As Single

Dim bestPref() As Single

Dim TotalNumObjFn As Single

TotalNumObjFn $=3$

Sheets("Parameters").Select

p1perprior $=$ ActiveSheet.Cells $(1,2)$.Value

p1perrestrict $=$ ActiveSheet.Cells $(2,2)$.Value

perprior $=$ ActiveSheet.Cells(3, 2).Value

perrestrict $=$ ActiveSheet.Cells $(4,2)$.Value

numiter $=$ ActiveSheet.Cells $(5,2)$.Value

phase1_on = 1 'this can be used as a switch to turn phase 1 on or off

Sheets("Input").Select

numworkers = ActiveSheet.Cells (2, 2).Value

numskills $=$ ActiveSheet.Cells $(3,2)$. Value

numtasks = ActiveSheet.Cells(4, 2).Value

numSolns $=$ ActiveSheet.Cells $(5,2)$.Value

'initialize arrays

ReDim workerskill(0 To numworkers + 1, 0 To numskills + 1) As Single ReDim oworkerskill( 0 To numworkers $+1,0$ To numskills +1$)$ As Single

ReDim taskskill $(0$ To numtasks $+1,0$ To numskills +1$)$ As Single

ReDim otaskskill(0 To numtasks $+1,0$ To numskills +1$)$ As Single

ReDim tasktime (0 To numtasks +1$)$ As Single

ReDim otasktime $(0$ To numtasks +1$)$ As Single 
ReDim workercapacity $(0$ To numworkers +1$)$ As Single

ReDim oworkercapacity (0 To numworkers +1$)$ As Single

ReDim traincost $(0$ To numskills $+1,0$ To 5, 0 To 5) As Single

ReDim otraincost( $(0$ To numskills $+1,0$ To 5,0 To 5) As Single

ReDim traintime(0 To numskills $+1,0$ To 5, 0 To 5) As Single

ReDim otraintime $(0$ To numskills $+1,0$ To 5, 0 To 5) As Single

ReDim workerassign $(0$ To 1, 0 To numworkers $+1,0$ To numtasks +1$)$ As Single

ReDim bestworkerassign $(0$ To numSolns $+1,0$ To numworkers $+1,0$ To numtasks +1 ) As Single

ReDim workertaskcost $(0$ To numworkers $+1,0$ To numtasks +1$)$ As Single

ReDim oworkertaskcost( 0 To numworkers $+1,0$ To numtasks +1$)$ As Single

ReDim workertaskSgap (0 To numworkers $+1,0$ To numtasks +1$)$ As Single

ReDim oworkertaskSgap (0 To numworkers $+1,0$ To numtasks +1$)$ As Single

ReDim workertaskPref $(0$ To numworkers $+1,0$ To numtasks +1$)$ As Single

ReDim oworkertaskPref( 0 To numworkers $+1,0$ To numtasks +1$)$ As Single

ReDim Prefmatrix $(0$ To numworkers $+1,0$ To numskills +1$)$ As Single

ReDim workertasktime $(0$ To numworkers $+1,0$ To numtasks +1$)$ As Single

ReDim oworkertasktime (0 To numworkers + 1, 0 To numtasks + 1) As Single

ReDim taskassigned(0 To numtasks +1$)$ As Single

ReDim tcost(0 To numskills $+1,0$ To 5) As Single

ReDim ttime (0 To numskills $+1,0$ To 5) As Single

ReDim available $(0$ To numworkers * numtasks $+1,0$ To 3$)$ As Single

ReDim totaltaskcost $(0$ To numtasks +1$)$ As Single

ReDim totalworkercost (0 To numworkers + 1) As Single

ReDim totaltaskSgap $(0$ To numtasks +1$)$ As Single

ReDim totalworkerSgap $(0$ To numworkers +1$)$ As Single

ReDim totaltaskPref(0 To numtasks +1$)$ As Single

ReDim totalworkerPref(0 To numworkers + 1) As Single

ReDim workerphase1(0 To numworkers + 1) As Single

ReDim trainingneeds $(0$ To numworkers $+1,0$ To numskills +1$)$ As Single

ReDim skilltrainingneeds( 0 To numskills $+1,0$ To 5) As Single

ReDim bestCost(0 To numSolns + 20) As Single 
ReDim bestSgap (0 To numSolns + 20) As Single ReDim bestPref(0 To numSolns + 20) As Single

starttime $=$ Timer

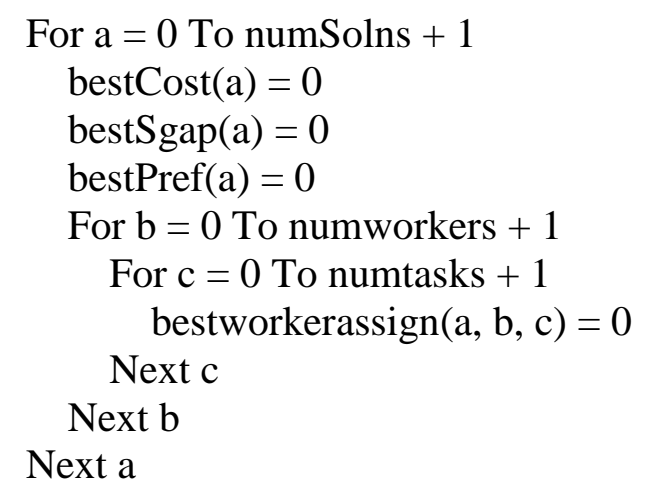

For $\mathrm{b}=0$ To numworkers +1

workercapacity $(b)=0$

oworkercapacity $(b)=0$

For $\mathrm{k}=0$ To numskills +1

workerskill( $(\mathrm{b}, \mathrm{k})=0$

oworkerskill $(\mathrm{b}, \mathrm{k})=0$

trainingneeds $(\mathrm{b}, \mathrm{k})=0$

Next k

Next b

For $\mathrm{b}=0$ To numworkers $*$ numtasks +1

For $\mathrm{k}=0$ To 3

available $(\mathrm{b}, \mathrm{k})=0$ 


\section{Next k}

\section{Next b}

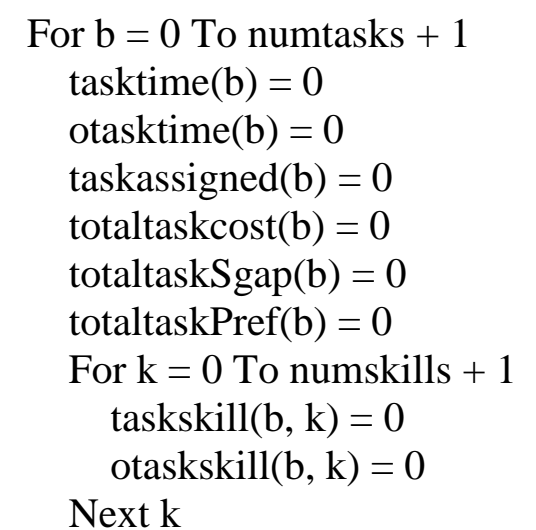

\section{Next b}

For $\mathrm{i}=0$ To numskills +1

For $\mathrm{j}=0$ To 5

$\operatorname{tcost}(i, j)=0$

time $(i, j)=0$

skilltrainingneeds $(i, j)=0$

For $\mathrm{k}=0$ To 5

traincost $(\mathrm{i}, \mathrm{j}, \mathrm{k})=0$

traintime $(\mathrm{i}, \mathrm{j}, \mathrm{k})=0$

otraincost $(\mathrm{i}, \mathrm{j}, \mathrm{k})=0$

otraintime $(i, j, k)=0$ Next k

Next j

Next $i$ 


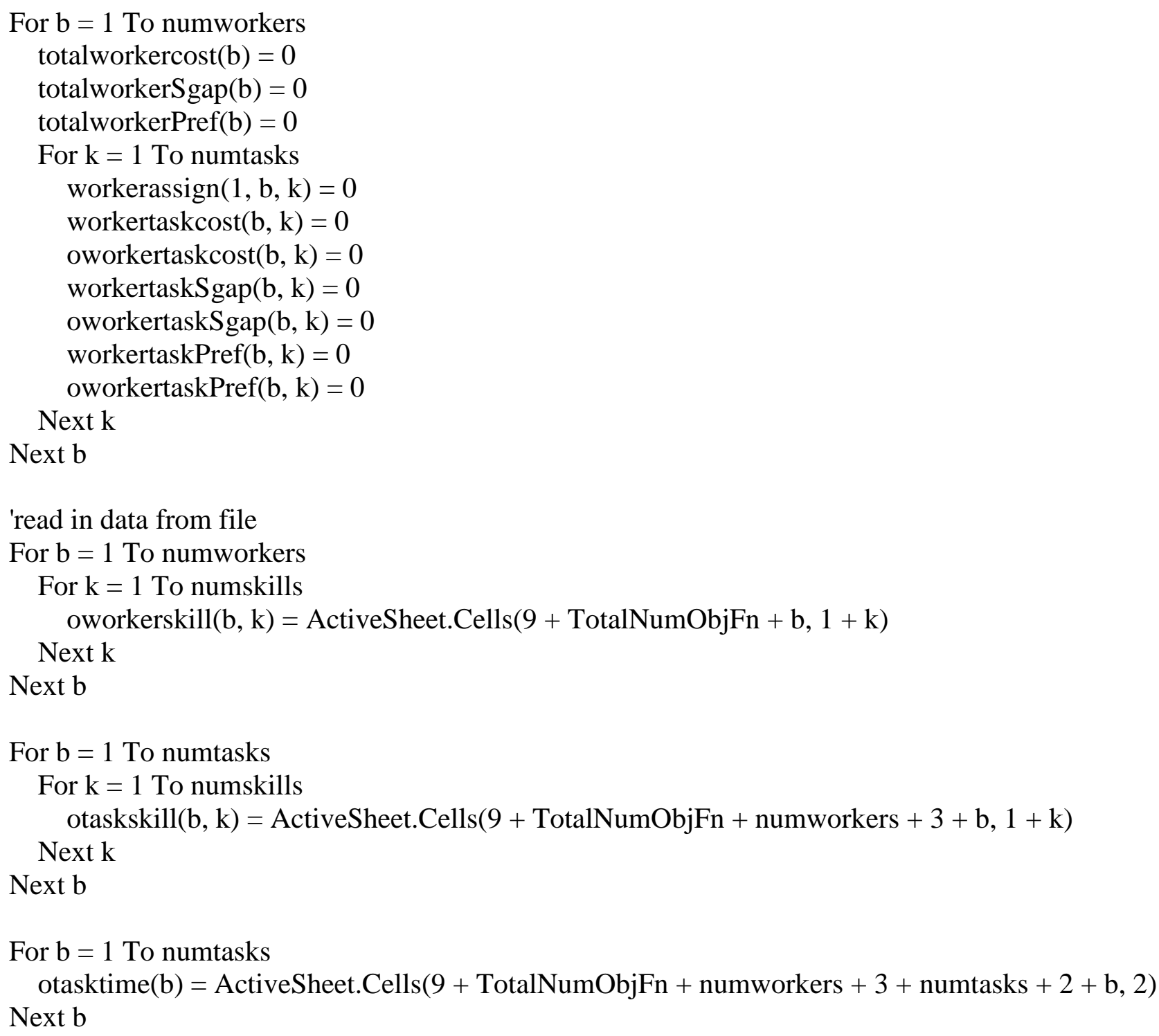


For $b=1$ To numworkers

oworkercapacity $(b)=$ ActiveSheet.Cells $(9+$ TotalNumObjFn + numworkers $+3+$ numtasks $+2+$ numtasks $+2+b, 2)$ Next b

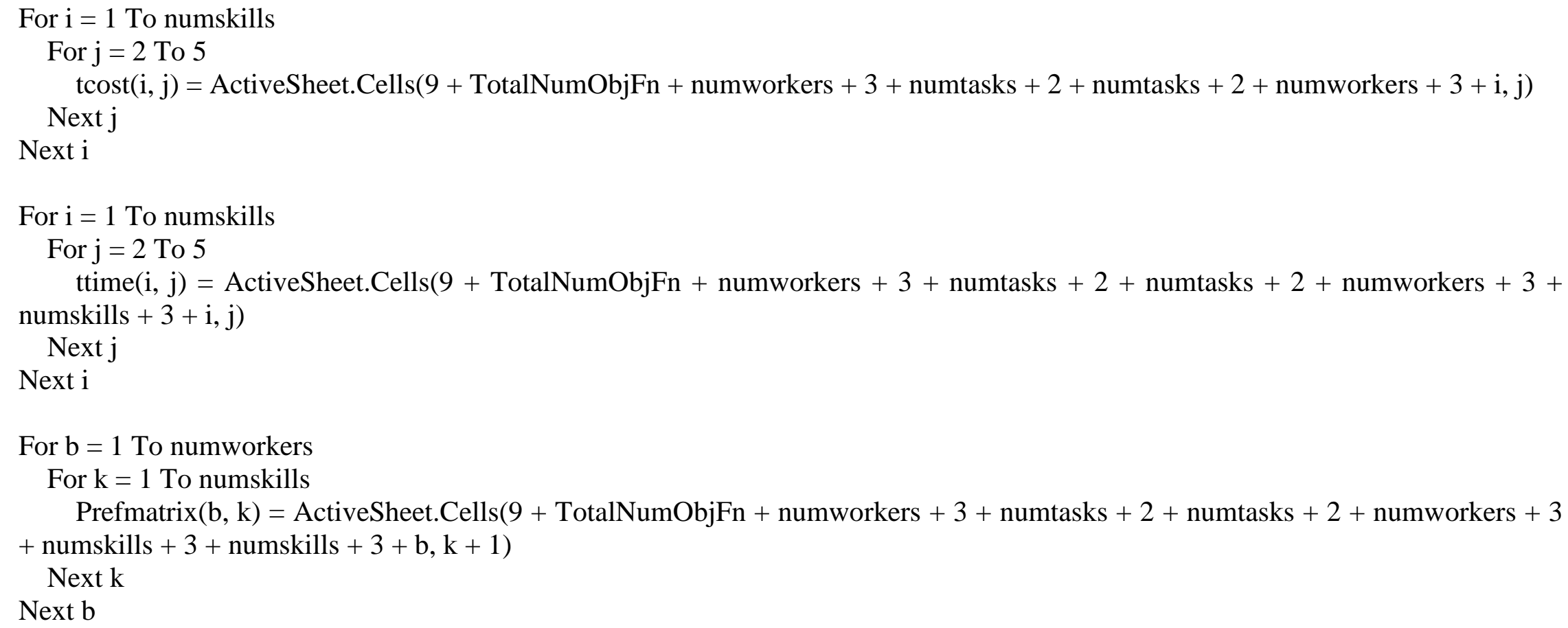


If $\mathrm{j}<\mathrm{k}$ And $\mathrm{k}>1$ Then

otraincost $(i, j, k)=\operatorname{otraincost}(i, j, k-1)+t \operatorname{cost}(i, k)$

End If

Next k

Next j

Next $\mathrm{i}$

For $\mathrm{i}=1$ To numskills

For $\mathrm{j}=1$ To 5

For $\mathrm{k}=1$ To 5

If $\mathrm{j}<\mathrm{k}$ And $\mathrm{k}>1$ Then

otraintime $(\mathrm{i}, \mathrm{j}, \mathrm{k})=$ otraintime $(\mathrm{i}, \mathrm{j}, \mathrm{k}-1)+\operatorname{ttime}(\mathrm{i}, \mathrm{k})$

End If

Next $k$

Next $j$

Next i

'find task cost and training time for each worker for each task

For $\mathrm{i}=1$ To numworkers

For $\mathrm{j}=1$ To numtasks

oworkertasktime $(i, j)=$ otasktime $(j)$

For $\mathrm{k}=1$ To numskills

If oworkerskill(i, k) < otaskskill(j, k) And otaskskill(j, k) > 1 Then

oworkertaskcost $(i, j)=$ oworkertaskcost $(i, j)+\operatorname{otraincost}(k$, oworkerskill $(i, k)$, otaskskill $(j, k))$

oworkertaskSgap $(i, j)=$ oworkertaskSgap $(i, j)+(\operatorname{otaskskill}(j, k)-$ oworkerskill $(i, k))$

oworkertaskPref $(i, j)=$ oworkertaskPref $(i, j)+\operatorname{Prefmatrix}(i, k) *($ otaskskill $(j, k)$ - oworkerskill $(i, k))$

oworkertasktime $(\mathrm{i}, \mathrm{j})=$ oworkertasktime $(\mathrm{i}, \mathrm{j})+\operatorname{otraintime}(\mathrm{k}$, oworkerskill $(\mathrm{i}, \mathrm{k})$, otaskskill $(\mathrm{j}, \mathrm{k}))$

End If

Next k

Next j 
Next i

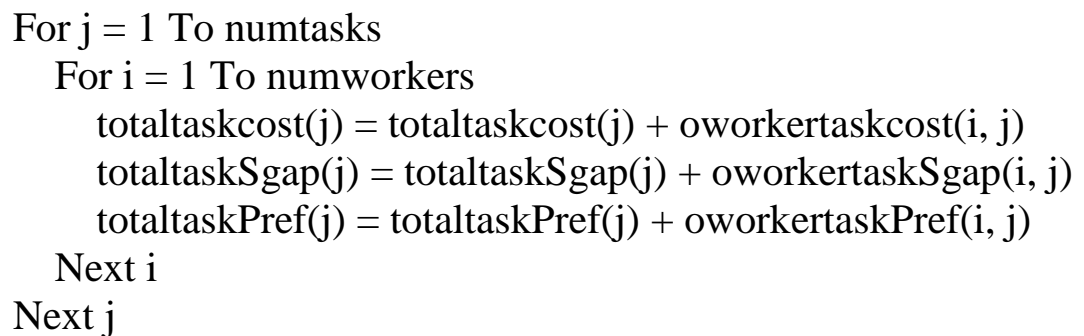

For $\mathrm{i}=1$ To numworkers

For $\mathrm{j}=1$ To numtasks

totalworkercost $(\mathrm{i})=$ totalworkercost $(\mathrm{i})+\operatorname{oworkertaskcost}(\mathrm{i}, \mathrm{j})$

totalworkerSgap $(\mathrm{i})=$ totalworkerSgap $(\mathrm{i})+\operatorname{oworkertaskSgap}(\mathrm{i}, \mathrm{j})$

totalworkerPref $(\mathrm{i})=$ totalworkerPref $(\mathrm{i})+$ oworkertaskPref $(\mathrm{i}, \mathrm{j})$

\section{Next $j$}

Next $\mathrm{i}$

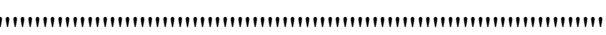

warned $3=0$

$$
\begin{aligned}
& \text { For a }=1 \text { To numSolns }+1 \\
& \text { bestCost }(\mathrm{a})=999999999 \\
& \text { bestSgap }(\mathrm{a})=-9999999 \\
& \text { bestPref(a) }=-9999999 \\
& \text { Next a } \\
& \text { bestCost }(0)=-9999999 \\
& \text { bestSgap }(0)=999999999 \\
& \text { bestPref }(0)=999999999
\end{aligned}
$$




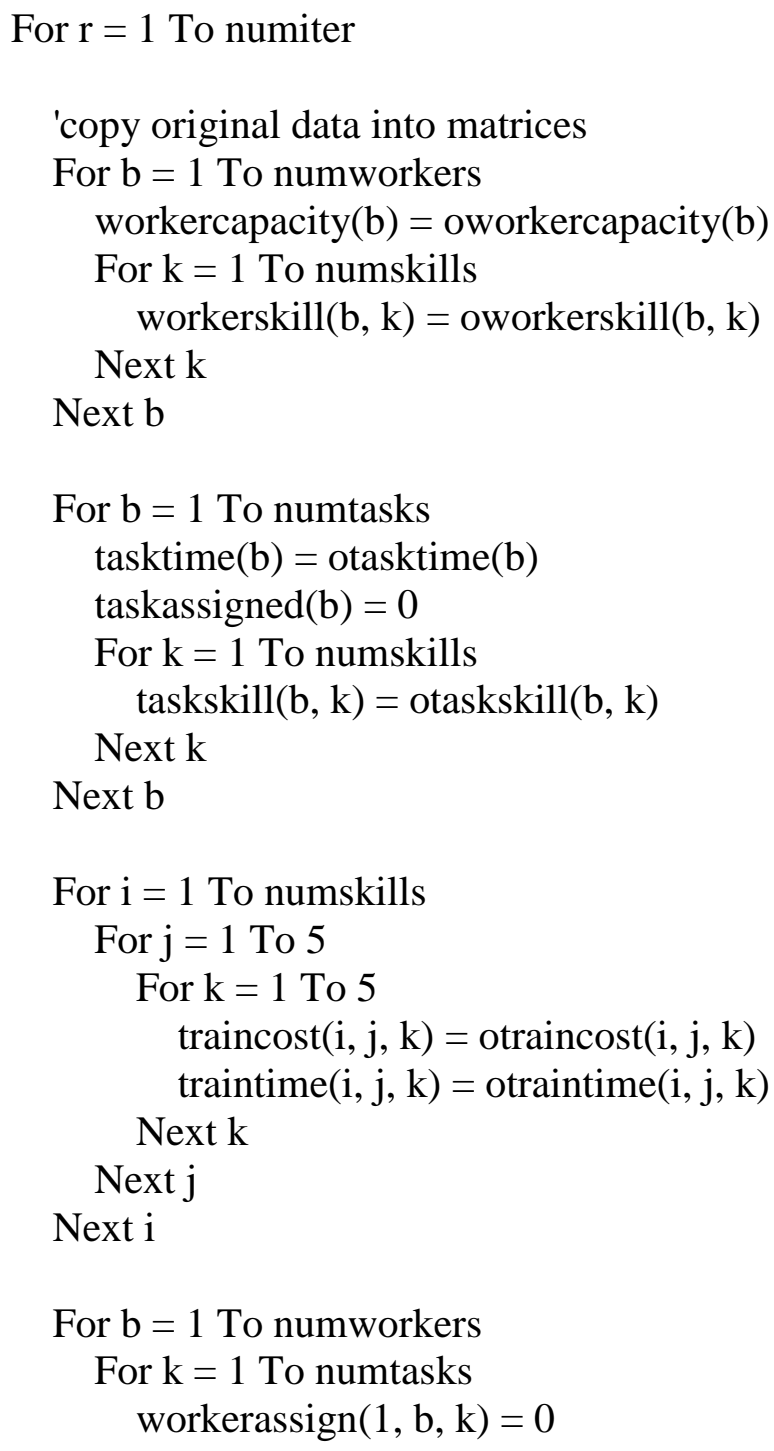


workertaskcost $(\mathrm{b}, \mathrm{k})=$ oworkertaskcost $(\mathrm{b}, \mathrm{k})$

workertaskSgap $(b, k)=$ oworkertaskSgap $(b, k)$

workertaskPref $(b, k)=$ oworkertaskPref $(b, k)$

workertasktime $(\mathrm{b}, \mathrm{k})=$ oworkertasktime $(\mathrm{b}, \mathrm{k})$

\section{Next k}

Next b

For $\mathrm{b}=1$ To numworkers

workerphase $1(b)=0$

Next b

$$
\begin{aligned}
& \text { totalcost }=0 \\
& \text { totalsgap }=0 \\
& \text { totalpref }=0 \\
& \text { numtaskassigned }=0
\end{aligned}
$$

If phase1_on $=1$ Then

Do While numtaskassigned < numworkers

maxsgapt $1=-9999999$

For $\mathrm{i}=1$ To numworkers

If workerphase $1(\mathrm{i})=0$ And totalworkerSgap(i) > maxsgapt 1 Then

maxsgapt $1=$ totalworkerSgap $(\mathrm{i})$

maxsgapworker $=\mathrm{i}$

$$
\text { End If }
$$

Next i

maxsgapt $2=-9999999$

For $\mathrm{j}=1$ To numtasks

If taskassigned $(\mathrm{j})=0$ And workertasktime(maxsgapworker, j) $<=$ workercapacity(maxsgapworker) And workertaskSgap(maxsgapworker, $\mathrm{j})>$ maxsgapt2 Then 

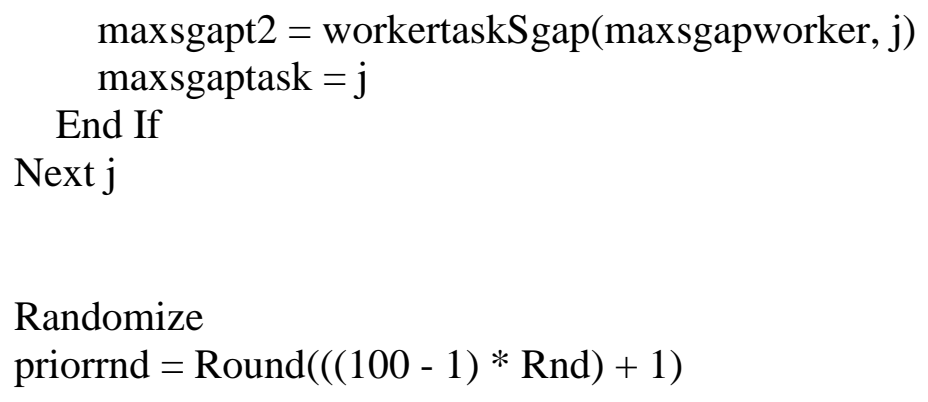

'make the best assignment

If priorrnd $<=$ p1 perprior Then

totalcost $=$ totalcost + workertaskcost (maxsgapworker, maxsgaptask)

totalsgap $=$ totalsgap + workertaskSgap (maxsgapworker, maxsgaptask $)$

totalpref $=$ totalpref + workertaskPref(maxsgapworker, maxsgaptask)

numtaskassigned $=$ numtaskassigned +1

workerassign $(1$, maxsgapworker, maxsgaptask $)=1$

taskassigned $($ maxsgaptask $)=1$

workerphase 1 (maxsgapworker $)=1$

workercapacity (maxsgapworker) = workercapacity (maxsgapworker) - workertasktime(maxsgapworker, maxsgaptask)

assignedworker $=$ maxsgapworker

assignedtask $=$ maxsgaptask

End If

If priorrnd > p1perprior Then

numonlist $=0$

For $\mathrm{i}=1$ To numworkers

If workerphase $1(\mathrm{i})=0$ And totalworkerSgap $(\mathrm{i})>=\operatorname{maxsgapt} 1 *(1-($ p1perrestrict $/ 100))$ Then

For $\mathrm{j}=1$ To numtasks

If taskassigned $(j)=0$ And workertaskSgap $(i, j)>=\operatorname{maxsgapt} 2 *(1-(p 1$ perrestrict $/ 100))$ And workertasktime $(i, j)$

$<=$ workercapacity(i) Then

numonlist $=$ numonlist +1 
available(numonlist, 1$)=\mathrm{i}$

available(numonlist, 2 ) $=\mathrm{j}$

End If

Next j

End If

Next i

\section{Randomize}

restrictrnd $=\operatorname{Round}((($ numonlist -1$) * \operatorname{Rnd})+1)$

assignedworker $=$ available $($ restrictrnd, 1$)$

assignedtask $=$ available (restrictrnd, 2 )

totalcost $=$ totalcost + workertaskcost $($ assignedworker, assignedtask $)$

totalsgap $=$ totalsgap + workertaskSgap(assignedworker, assignedtask)

totalpref $=$ totalpref + workertaskPref(assignedworker, assignedtask)

numtaskassigned $=$ numtaskassigned +1

workerassign $(1$, assignedworker, assignedtask $)=1$

taskassigned(assignedtask $)=1$

workerphase 1 (assignedworker $)=1$

workercapacity(assignedworker) = workercapacity(assignedworker) - workertasktime(assignedworker, assignedtask) End If

For $\mathrm{k}=1$ To numskills

If workerskill(assignedworker, $\mathrm{k}$ ) < taskskill(assignedtask, $\mathrm{k}$ ) Then workerskill(assignedworker, $\mathrm{k}$ ) = taskskill(assignedtask, $\mathrm{k}$ )

End If

Next k

For $\mathrm{j}=1$ To numtasks 
If taskassigned $(j)=0$ Then

workertaskcost(assignedworker, $\mathrm{j})=0$

workertaskSgap(assignedworker, $\mathrm{j}$ ) $=0$

workertaskPref(assignedworker, $\mathrm{j})=0$

workertasktime (assignedworker, $\mathrm{j})=$ otasktime $(\mathrm{j})$

For $\mathrm{k}=1$ To numskills

If workerskill(assignedworker, $\mathrm{k})<\operatorname{taskskill(j,k)}$ And taskskill(j, $\mathrm{k})>1$ Then

k), taskskill(j, k))

workertaskcost $($ assignedworker, $\mathrm{j})=$ workertaskcost $($ assignedworker, $\mathrm{j})+\operatorname{traincost}(\mathrm{k}$, workerskill(assignedworker,

workertaskSgap(assignedworker, j) $=$ workertaskSgap(assignedworker, j) $\quad+\quad(\operatorname{taskskill}(\mathrm{j}, \quad \mathrm{k}) \quad-$ workerskill(assignedworker, k))

workertaskPref(assignedworker, $\mathrm{j})=$ workertaskPref(assignedworker, $\mathrm{j})+\operatorname{Prefmatrix}(\operatorname{assignedworker,~k)*}$ (taskskill(j, k) - workerskill(assignedworker, k))

workertasktime (assignedworker, $\mathrm{j})=$ workertasktime $($ assignedworker, $\quad \mathrm{j}) \quad+\quad \operatorname{traintime}(\mathrm{k}$,

workerskill(assignedworker, k), taskskill(j, k))

End If

Next k

End If

Next j

\section{Loop}

End If 'If phase1_on $=1$

'end of phase 1 switch

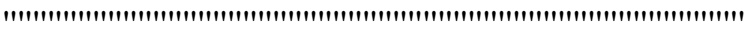

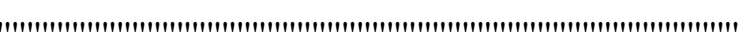

Do While numtaskassigned < numtasks 'repeat until all tasks assigned maxsgapt1 $=-9999999$ 
For $\mathrm{j}=1$ To numtasks

If taskassigned $(\mathrm{j})=0$ And totaltaskSgap $(\mathrm{j})>\operatorname{maxsgapt} 1$ Then

maxsgapt $1=$ totaltaskSgap $(\mathrm{j})$

maxsgaptask $=\mathrm{j}$

End If

Next j

maxsgapt2 $=-9999999$

maxsgapworker $=0$

For $\mathrm{i}=1$ To numworkers

If workertasktime(i, maxsgaptask) $<=$ workercapacity(i) And workertaskSgap(i, maxsgaptask) > maxsgapt2 Then maxsgapt $2=$ workertaskSgap (i, maxsgaptask)

maxsgapworker $=\mathrm{i}$

End If

Next i

\section{Randomize}

priorrnd $=\operatorname{Round}(((100-1) *$ Rnd $)+1)$

If maxsgapworker $>0$ Then

If priorrnd $<=$ perprior Then

totalcost $=$ totalcost + workertaskcost $($ maxsgapworker, maxsgaptask $)$

totalsgap $=$ totalsgap + workertaskSgap $($ maxsgapworker, maxsgaptask $)$

totalpref $=$ totalpref + workertaskPref(maxsgapworker, maxsgaptask $)$

numtaskassigned $=$ numtaskassigned +1

workerassign $(1$, maxsgapworker, maxsgaptask $)=1$

taskassigned $($ maxsgaptask $)=1$

workercapacity (maxsgapworker) = workercapacity(maxsgapworker) - workertasktime (maxsgapworker, maxsgaptask) assignedworker $=$ maxsgapworker 
assignedtask $=$ maxsgaptask

End If

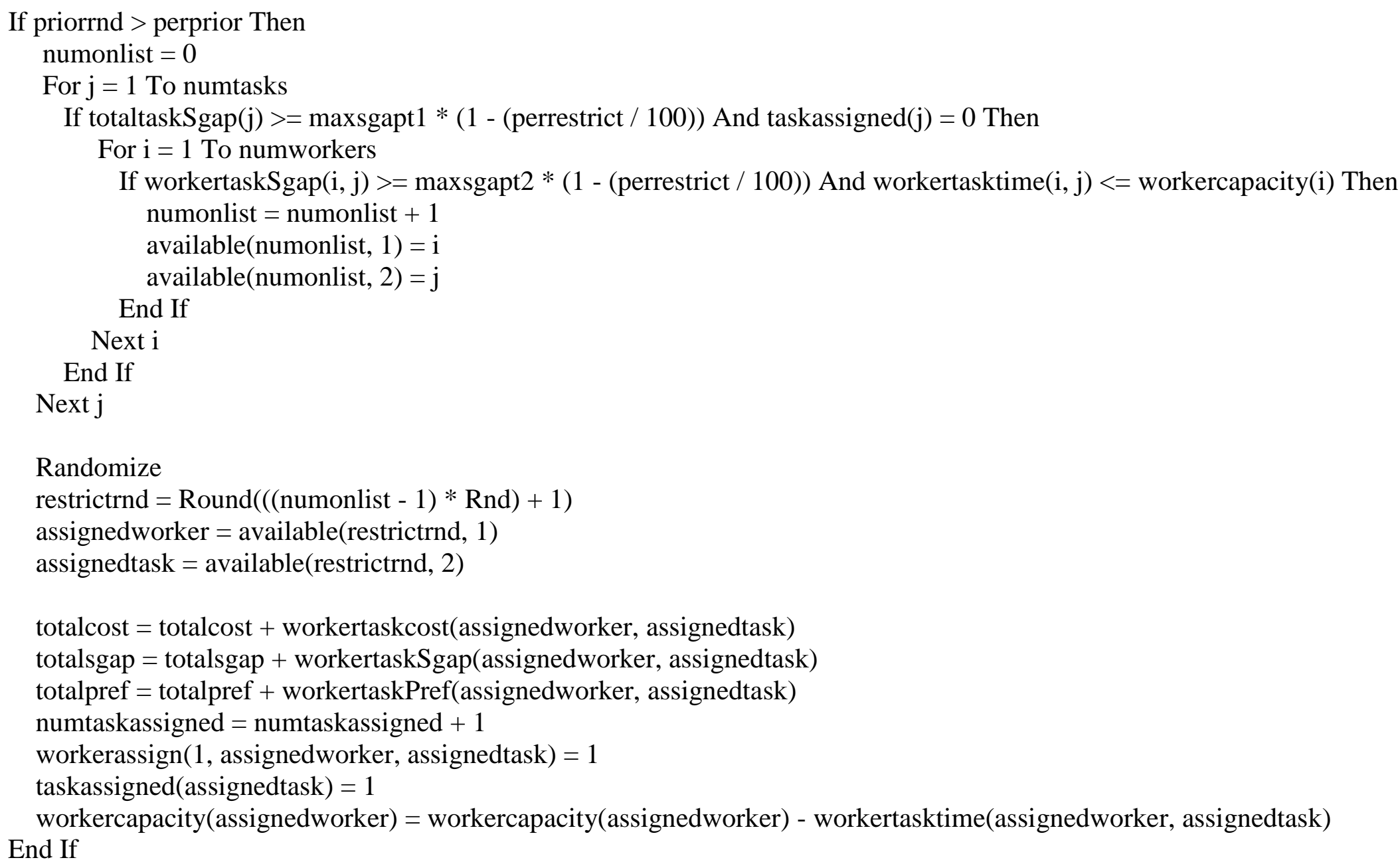


For $\mathrm{k}=1$ To numskills

If workerskill(assignedworker, k) < taskskill(assignedtask, k) Then

workerskill(assignedworker, $\mathrm{k}$ ) = taskskill(assignedtask, $\mathrm{k}$ )

End If

Next k

For $\mathrm{j}=1$ To numtasks

If taskassigned $(j)=0$ Then

workertaskcost $($ assignedworker, $\mathrm{j})=0$

workertaskSgap(assignedworker, $\mathrm{j}$ ) $=0$

workertaskPref(assignedworker, $\mathrm{j}$ ) $=0$

workertasktime(assignedworker, $\mathrm{j})=$ otasktime $(\mathrm{j})$

For $\mathrm{k}=1$ To numskills

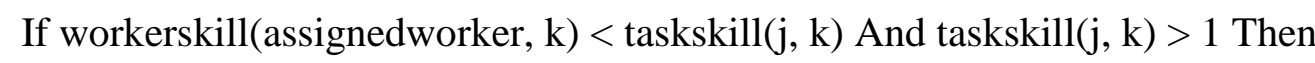

$\mathrm{k})$, taskskill( $(\mathrm{j}, \mathrm{k}))$

workertaskcost(assignedworker, $\mathrm{j})=$ workertaskcost(assignedworker, $\mathrm{j})+\operatorname{traincost}(\mathrm{k}$, workerskill(assignedworker,

workertaskSgap(assignedworker, j) = workertaskSgap(assignedworker, j) $\quad+\quad(\operatorname{taskskill}(\mathrm{j}, \quad \mathrm{k}) \quad-$ workerskill(assignedworker, $\mathrm{k})$ )

workertaskPref(assignedworker, j) = workertaskPref(assignedworker, j) + Prefmatrix(assignedworker, k) * (taskskill(j, k) - workerskill(assignedworker, k))

k), taskskill(j, k))

workertasktime $($ assignedworker, $\mathrm{j})=$ workertasktime $($ assignedworker, $\mathrm{j})+\operatorname{traintime}(\mathrm{k}$, workerskill $($ assignedworker,

$$
\begin{aligned}
& \text { End If } \\
& \text { Next } \mathrm{k} \\
& \text { End If } \\
& \text { Next } \mathrm{j}
\end{aligned}
$$

End If

If maxsgapworker $=0$ Then 


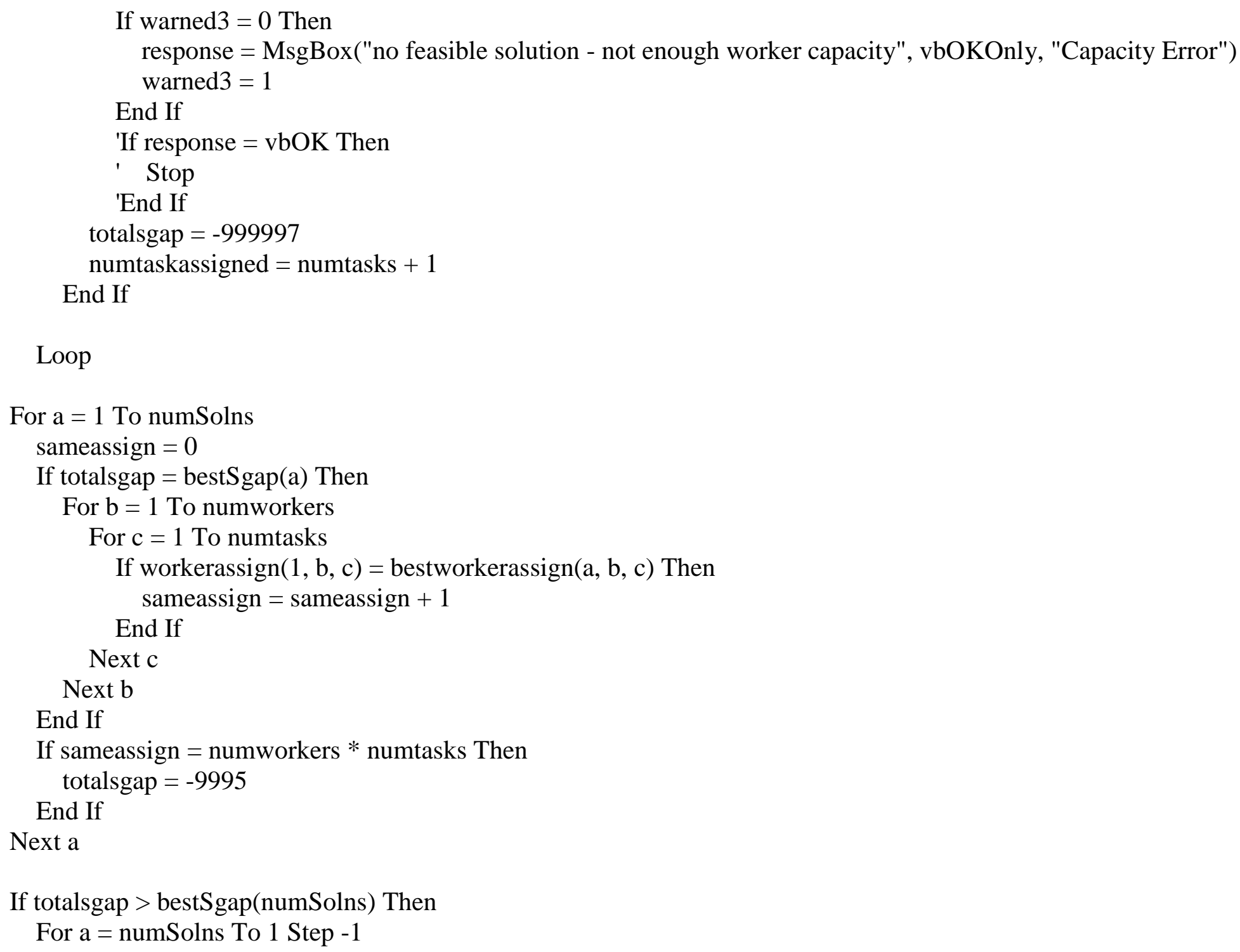




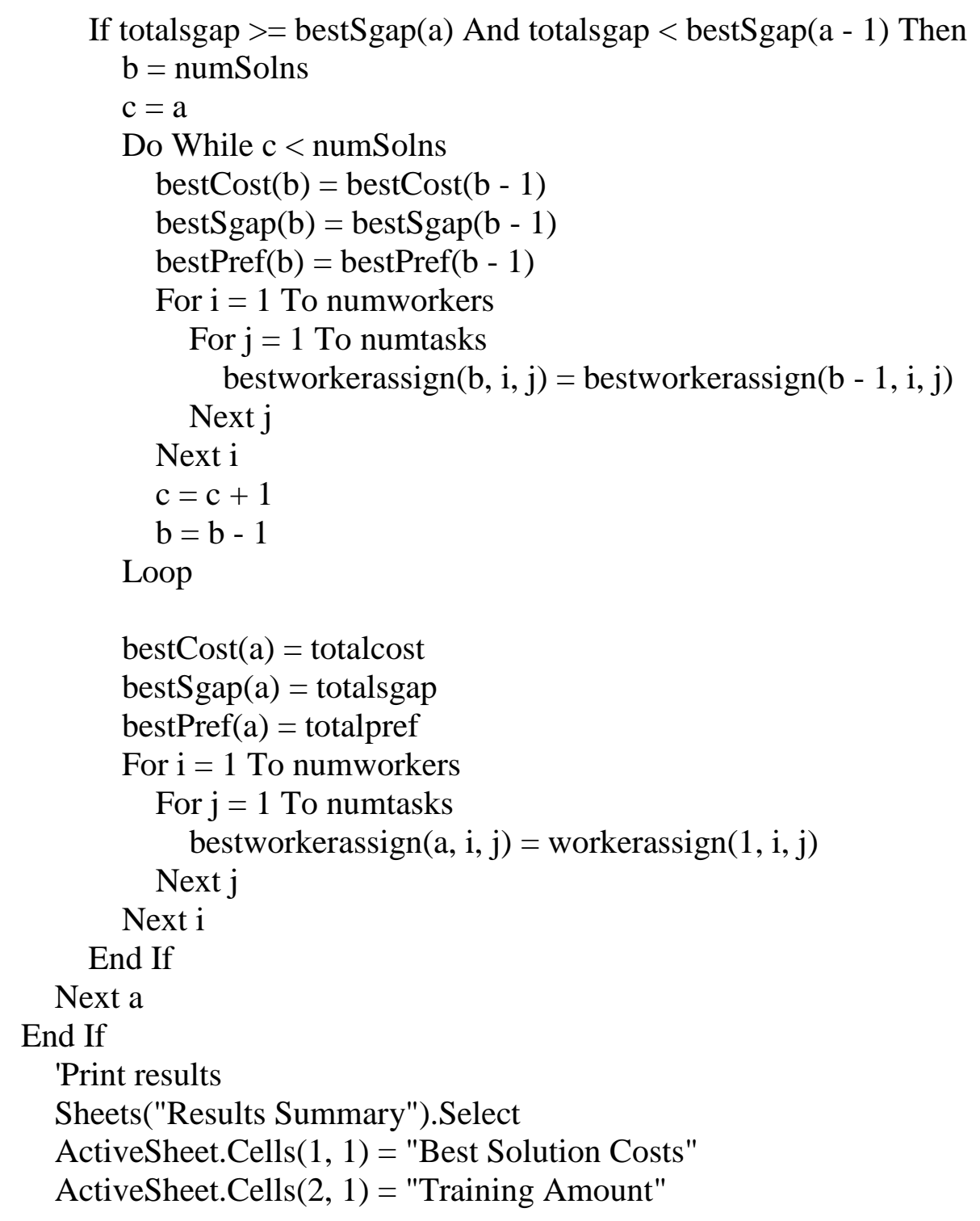


ActiveSheet.Cells $(3,1)=$ "Worker Preference"

For $\mathrm{a}=1$ To numSolns

ActiveSheet.Cells $(1, a+1)=$ bestCost(a)

Next a

For $\mathrm{a}=1$ To numSolns

$\operatorname{ActiveSheet} \cdot \operatorname{Cells}(2, a+1)=\operatorname{bestSgap}(a)$

Next a

For $\mathrm{a}=1$ To numSolns

ActiveSheet.Cells $(3, a+1)=\operatorname{bestPref}(a)$

Next a

Next $r$

endtime $=$ Timer

totaltime $=$ endtime - starttime

Sheets("Results Summary").Select

ActiveSheet.Cells $(2$, numSolns +3$)=$ "Run Time"

ActiveSheet.Cells $(2$, numSolns +4$)=$ totaltime

'Print to Results Summary sheet

Sheets("Results Summary").Select

'ActiveSheet.Cells $(1,1)=$ "Best Solution Costs"

'ActiveSheet.Cells $(1,2)=$ bestsolution $(1)$

ActiveSheet.Cells(12 + numtasks, 1) = "Solution \#:"

For $\mathrm{a}=1$ To numSolns

ActiveSheet.Cells $(12+$ numtasks, $2 *$ a $)=$ numSolns + a

Next a

$\mathrm{b}=1$

For $\mathrm{a}=1$ To numSolns $* 2$ 
ActiveSheet.Cells $(13+$ numtasks, a $)=$ "Worker"

ActiveSheet.Cells $(13+$ numtasks, $\mathrm{a}+1)=$ "Task"

ActiveSheet.Cells $(14+2 *$ numtasks, 1$)=$ "Total Cost"

ActiveSheet.Cells $(15+2 *$ numtasks, 1$)=$ "Total Skill Levels"

ActiveSheet.Cells $(16+2 *$ numtasks, 1$)=$ "Total Preference"

cellrow $=14+$ numtasks

For $\mathrm{i}=1$ To numworkers

For $\mathrm{j}=1$ To numtasks

If bestworkerassign $(b, i, j)=1$ Then

ActiveSheet.Cells (cellrow, a) $=\mathrm{i}$

ActiveSheet.Cells (cellrow, $\mathrm{a}+1)=\mathrm{j}$

cellrow $=$ cellrow +1

End If

Next $\mathrm{j}$

Next i

ActiveSheet.Cells $(14+2 *$ numtasks, $\mathrm{a}+1)=$ bestCost $(\mathrm{b})$

ActiveSheet.Cells $(15+2 *$ numtasks, $a+1)=$ bestSgap $(b)$

ActiveSheet.Cells $(16+2 *$ numtasks, $a+1)=\operatorname{bestPref}(b)$

$\mathrm{b}=\mathrm{b}+1$

$\mathrm{a}=\mathrm{a}+1$

Next a

\section{End Sub}


Public Sub InitialWorkerPrefSoln_click()

Dim workerskill() As Single, oworkerskill() As Single

Dim taskskill() As Single, otaskskill() As Single

Dim tasktime() As Single, otasktime() As Single

Dim workercapacity() As Single, oworkercapacity() As Single

Dim traincost() As Single, otraincost() As Single

Dim traintime() As Single, otraintime() As Single

Dim workerassign() As Single

Dim workertaskcost() As Single, oworkertaskcost() As Single

Dim workertasktime() As Single, oworkertasktime() As Single

Dim workertaskSgap() As Single, oworkertaskSgap() As Single

Dim workertaskPref() As Single, oworkertaskPref() As Single

Dim Prefmatrix() As Single

Dim taskassigned() As Single

Dim tcost() As Single

Dim ttime() As Single

Dim available() As Single

Dim bestworkerassign() As Single

Dim numworkers As Single, numskills As Single, numtasks As Single, numSolns As Single

Dim totaltaskcost() As Single

Dim totalworkercost() As Single

Dim totaltaskSgap() As Single

Dim totalworkerSgap() As Single

Dim totaltaskPref() As Single

Dim totalworkerPref() As Single

Dim workerphase1() As Single

Dim cellrow As Single

Dim trainingneeds() As Single

Dim skilltrainingneeds() As Single

Dim bestCost() As Single 
Dim bestSgap() As Single

Dim bestPref() As Single

Dim TotalNumObjFn As Single

TotalNumObjFn $=3$

Sheets("Parameters").Select

p1 perprior $=$ ActiveSheet.Cells $(1,2)$. Value

p1perrestrict $=$ ActiveSheet.Cells $(2,2)$.Value

perprior $=$ ActiveSheet.Cells(3, 2).Value

perrestrict $=$ ActiveSheet.Cells $(4,2)$.Value

numiter $=$ ActiveSheet.Cells $(5,2)$.Value

phase1_on = 1 'this can be used as a switch to turn phase 1 on or off

Sheets("Input").Select

numworkers = ActiveSheet.Cells (2, 2).Value

numskills $=$ ActiveSheet.Cells $(3,2)$. Value

numtasks $=$ ActiveSheet.Cells $(4,2)$.Value

numSolns $=$ ActiveSheet.Cells $(5,2)$.Value

'initialize arrays

ReDim workerskill(0 To numworkers + 1, 0 To numskills + 1) As Single ReDim oworkerskill( 0 To numworkers $+1,0$ To numskills +1$)$ As Single

ReDim taskskill $(0$ To numtasks $+1,0$ To numskills +1$)$ As Single

ReDim otaskskill(0 To numtasks $+1,0$ To numskills +1$)$ As Single

ReDim tasktime (0 To numtasks +1$)$ As Single

ReDim otasktime $(0$ To numtasks +1$)$ As Single 
ReDim workercapacity $(0$ To numworkers +1$)$ As Single

ReDim oworkercapacity (0 To numworkers +1$)$ As Single

ReDim traincost $(0$ To numskills $+1,0$ To 5, 0 To 5) As Single

ReDim otraincost( $(0$ To numskills $+1,0$ To 5,0 To 5$)$ As Single

ReDim traintime(0 To numskills $+1,0$ To 5, 0 To 5) As Single

ReDim otraintime $(0$ To numskills $+1,0$ To 5, 0 To 5) As Single

ReDim workerassign $(0$ To 1, 0 To numworkers $+1,0$ To numtasks +1$)$ As Single

ReDim bestworkerassign $(0$ To numSolns $+1,0$ To numworkers $+1,0$ To numtasks +1 ) As Single

ReDim workertaskcost $(0$ To numworkers $+1,0$ To numtasks +1$)$ As Single

ReDim oworkertaskcost $(0$ To numworkers $+1,0$ To numtasks + 1) As Single

ReDim workertaskSgap (0 To numworkers + 1, 0 To numtasks + 1) As Single

ReDim oworkertaskSgap $(0$ To numworkers $+1,0$ To numtasks +1$)$ As Single

ReDim workertaskPref $(0$ To numworkers $+1,0$ To numtasks +1$)$ As Single

ReDim oworkertaskPref( 0 To numworkers $+1,0$ To numtasks +1$)$ As Single

ReDim Prefmatrix $(0$ To numworkers $+1,0$ To numskills +1 ) As Single

ReDim workertasktime (0 To numworkers $+1,0$ To numtasks + 1) As Single

ReDim oworkertasktime (0 To numworkers + 1, 0 To numtasks + 1) As Single

ReDim taskassigned(0 To numtasks +1$)$ As Single

ReDim tcost(0 To numskills $+1,0$ To 5) As Single

ReDim ttime (0 To numskills $+1,0$ To 5) As Single

ReDim available $(0$ To numworkers * numtasks $+1,0$ To 3$)$ As Single

ReDim totaltaskcost $(0$ To numtasks +1$)$ As Single

ReDim totalworkercost (0 To numworkers + 1) As Single

ReDim totaltaskSgap $(0$ To numtasks +1$)$ As Single

ReDim totalworkerSgap $(0$ To numworkers +1$)$ As Single

ReDim totaltaskPref(0 To numtasks + 1) As Single

ReDim totalworkerPref( 0 To numworkers +1$)$ As Single

ReDim workerphase1(0 To numworkers + 1) As Single

ReDim trainingneeds $(0$ To numworkers $+1,0$ To numskills +1$)$ As Single

ReDim skilltrainingneeds(0 To numskills $+1,0$ To 5) As Single

ReDim bestCost(0 To numSolns + 20) As Single 
ReDim bestSgap (0 To numSolns + 20) As Single ReDim bestPref(0 To numSolns + 20) As Single

starttime $=$ Timer

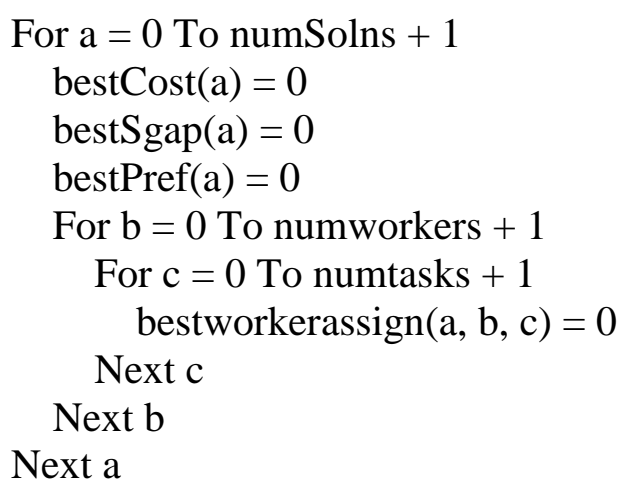

For $\mathrm{b}=0$ To numworkers +1

workercapacity $(b)=0$

oworkercapacity $(b)=0$

For $\mathrm{k}=0$ To numskills +1

workerskill( $(\mathrm{b}, \mathrm{k})=0$

oworkerskill $(\mathrm{b}, \mathrm{k})=0$

trainingneeds $(\mathrm{b}, \mathrm{k})=0$

Next k

Next b

For $\mathrm{b}=0$ To numworkers $*$ numtasks +1

For $\mathrm{k}=0$ To 3

available $(\mathrm{b}, \mathrm{k})=0$ 


\section{Next k}

\section{Next b}

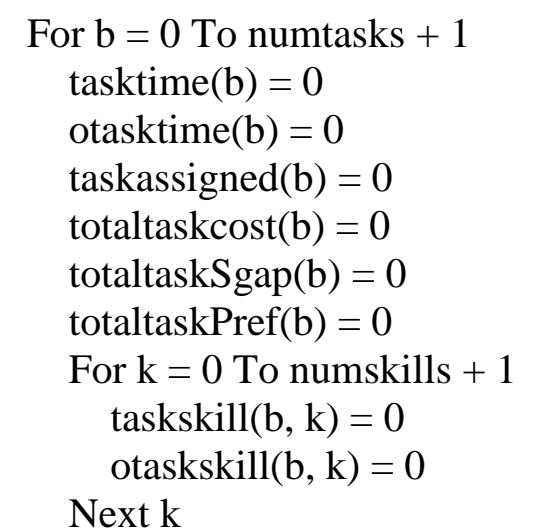

\section{Next b}

For $\mathrm{i}=0$ To numskills +1

For $\mathrm{j}=0$ To 5

$\operatorname{tcost}(i, j)=0$

time $(i, j)=0$

skilltrainingneeds $(i, j)=0$

For $\mathrm{k}=0$ To 5

traincost $(\mathrm{i}, \mathrm{j}, \mathrm{k})=0$

traintime $(\mathrm{i}, \mathrm{j}, \mathrm{k})=0$

otraincost $(\mathrm{i}, \mathrm{j}, \mathrm{k})=0$

otraintime $(i, j, k)=0$ Next k

Next j

Next $i$ 





For $b=1$ To numworkers

oworkercapacity $(b)=$ ActiveSheet.Cells $(9+$ TotalNumObjFn + numworkers $+3+$ numtasks $+2+$ numtasks $+2+b, 2)$ Next b

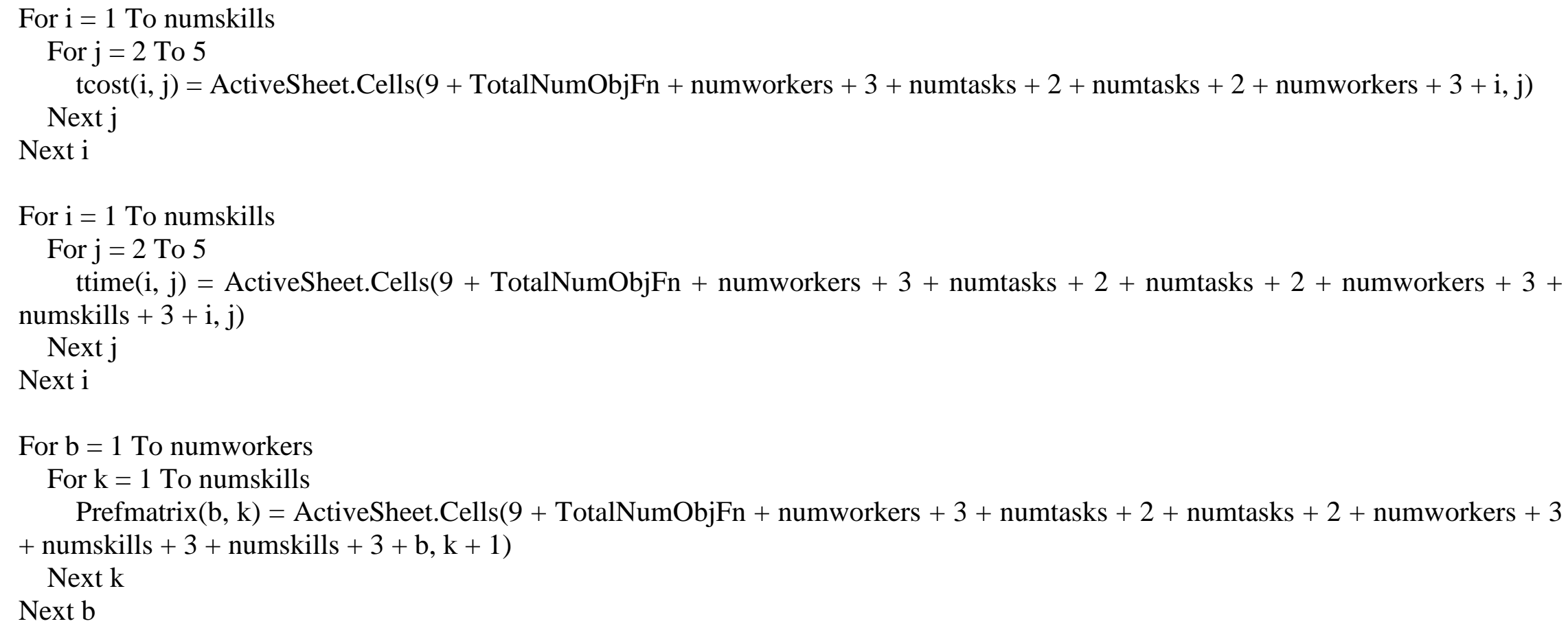


If $\mathrm{j}<\mathrm{k}$ And $\mathrm{k}>1$ Then

otraincost $(i, j, k)=\operatorname{otraincost}(i, j, k-1)+t \operatorname{cost}(i, k)$

End If

Next k

Next j

Next i

For $\mathrm{i}=1$ To numskills

For $\mathrm{j}=1$ To 5

For $\mathrm{k}=1$ To 5

If $\mathrm{j}<\mathrm{k}$ And $\mathrm{k}>1$ Then

otraintime $(\mathrm{i}, \mathrm{j}, \mathrm{k})=$ otraintime $(\mathrm{i}, \mathrm{j}, \mathrm{k}-1)+\operatorname{ttime}(\mathrm{i}, \mathrm{k})$

End If

Next $k$

Next $j$

Next i

'find task cost and training time for each worker for each task

For $\mathrm{i}=1$ To numworkers

For $\mathrm{j}=1$ To numtasks

oworkertasktime $(i, j)=$ otasktime $(j)$

For $\mathrm{k}=1$ To numskills

If oworkerskill(i, k) < otaskskill(j, k) And otaskskill(j, k) > 1 Then

oworkertaskcost $(i, j)=$ oworkertaskcost $(i, j)+\operatorname{otraincost}(k$, oworkerskill $(i, k)$, otaskskill $(j, k))$

oworkertaskSgap $(i, j)=$ oworkertaskSgap $(i, j)+(\operatorname{otaskskill}(j, k)-$ oworkerskill $(i, k))$

oworkertaskPref $(\mathrm{i}, \mathrm{j})=\operatorname{oworkertaskPref}(\mathrm{i}, \mathrm{j})+\operatorname{Prefmatrix}(\mathrm{i}, \mathrm{k}) *(\operatorname{otaskskill}(\mathrm{j}, \mathrm{k})$ - oworkerskill(i, k))

oworkertasktime $(\mathrm{i}, \mathrm{j})=$ oworkertasktime $(\mathrm{i}, \mathrm{j})+\operatorname{otraintime}(\mathrm{k}$, oworkerskill $(\mathrm{i}, \mathrm{k})$, otaskskill $(\mathrm{j}, \mathrm{k}))$

End If

Next k

Next j 
Next i

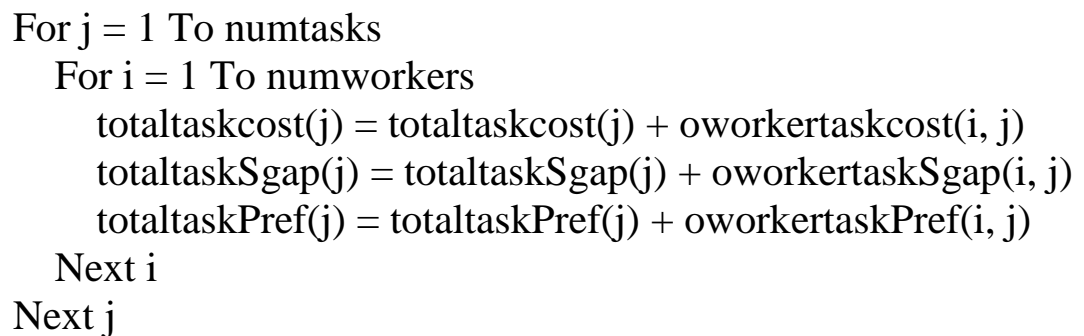

For $\mathrm{i}=1$ To numworkers

For $\mathrm{j}=1$ To numtasks

totalworkercost $(\mathrm{i})=$ totalworkercost $(\mathrm{i})+\operatorname{oworkertaskcost}(\mathrm{i}, \mathrm{j})$

totalworkerSgap $(\mathrm{i})=$ totalworkerSgap $(\mathrm{i})+\operatorname{oworkertaskSgap}(\mathrm{i}, \mathrm{j})$

totalworkerPref $(\mathrm{i})=$ totalworkerPref $(\mathrm{i})+$ oworkertaskPref $(\mathrm{i}, \mathrm{j})$

\section{Next $j$}

Next $\mathrm{i}$

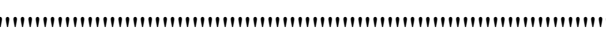

warned $4=0$

$$
\begin{aligned}
& \text { For a }=1 \text { To numSolns }+1 \\
& \text { bestCost }(\mathrm{a})=999999999 \\
& \text { bestSgap }(\mathrm{a})=-9999999 \\
& \text { bestPref(a) }=-9999999 \\
& \text { Next a } \\
& \text { bestCost }(0)=-9999999 \\
& \text { bestSgap }(0)=999999999 \\
& \text { bestPref }(0)=999999999
\end{aligned}
$$




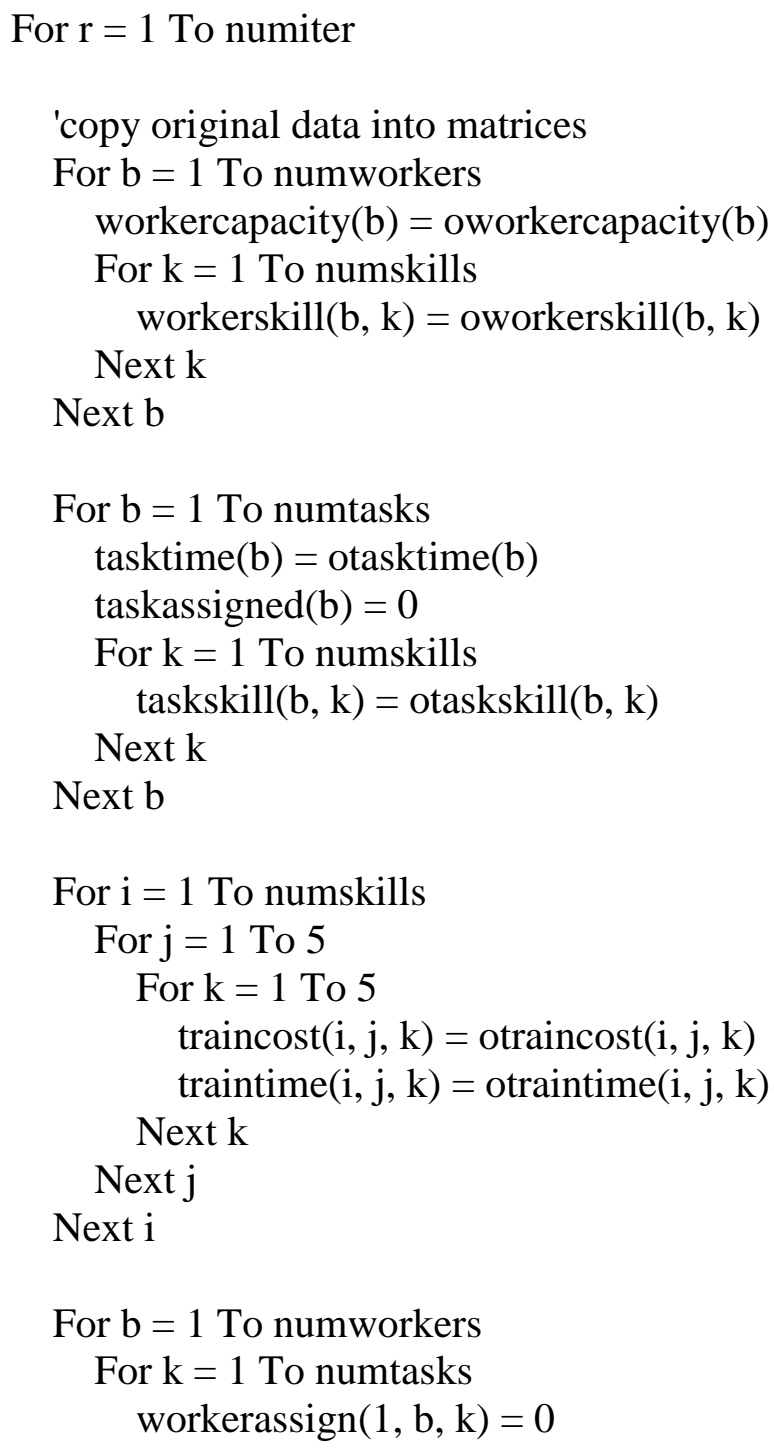


workertaskcost $(\mathrm{b}, \mathrm{k})=$ oworkertaskcost $(\mathrm{b}, \mathrm{k})$

workertaskSgap $(b, k)=$ oworkertaskSgap $(b, k)$

workertaskPref $(b, k)=$ oworkertaskPref $(b, k)$

workertasktime $(\mathrm{b}, \mathrm{k})=$ oworkertasktime $(\mathrm{b}, \mathrm{k})$

\section{Next k}

Next b

For $\mathrm{b}=1$ To numworkers

workerphase $1(b)=0$

Next b

$$
\begin{aligned}
& \text { totalcost }=0 \\
& \text { totalsgap }=0 \\
& \text { totalpref }=0 \\
& \text { numtaskassigned }=0
\end{aligned}
$$

If phase1_on = 1 Then

Do While numtaskassigned $<$ numworkers

maxpreft $1=-9999999$

For $\mathrm{i}=1$ To numworkers

If workerphase $1(\mathrm{i})=0$ And totalworkerPref(i) $>$ maxpreft1 Then

maxpreft $1=$ totalworkerPref(i)

maxprefworker $=\mathrm{i}$

$$
\text { End If }
$$

Next i

maxpreft $2=-9999999$

For $\mathrm{j}=1$ To numtasks

If taskassigned(j) $=0$ And workertasktime(maxprefworker, $\mathrm{j}$ ) $<=$ workercapacity(maxprefworker) And workertaskPref(maxprefworker, $\mathrm{j})>\operatorname{maxpreft} 2$ Then 


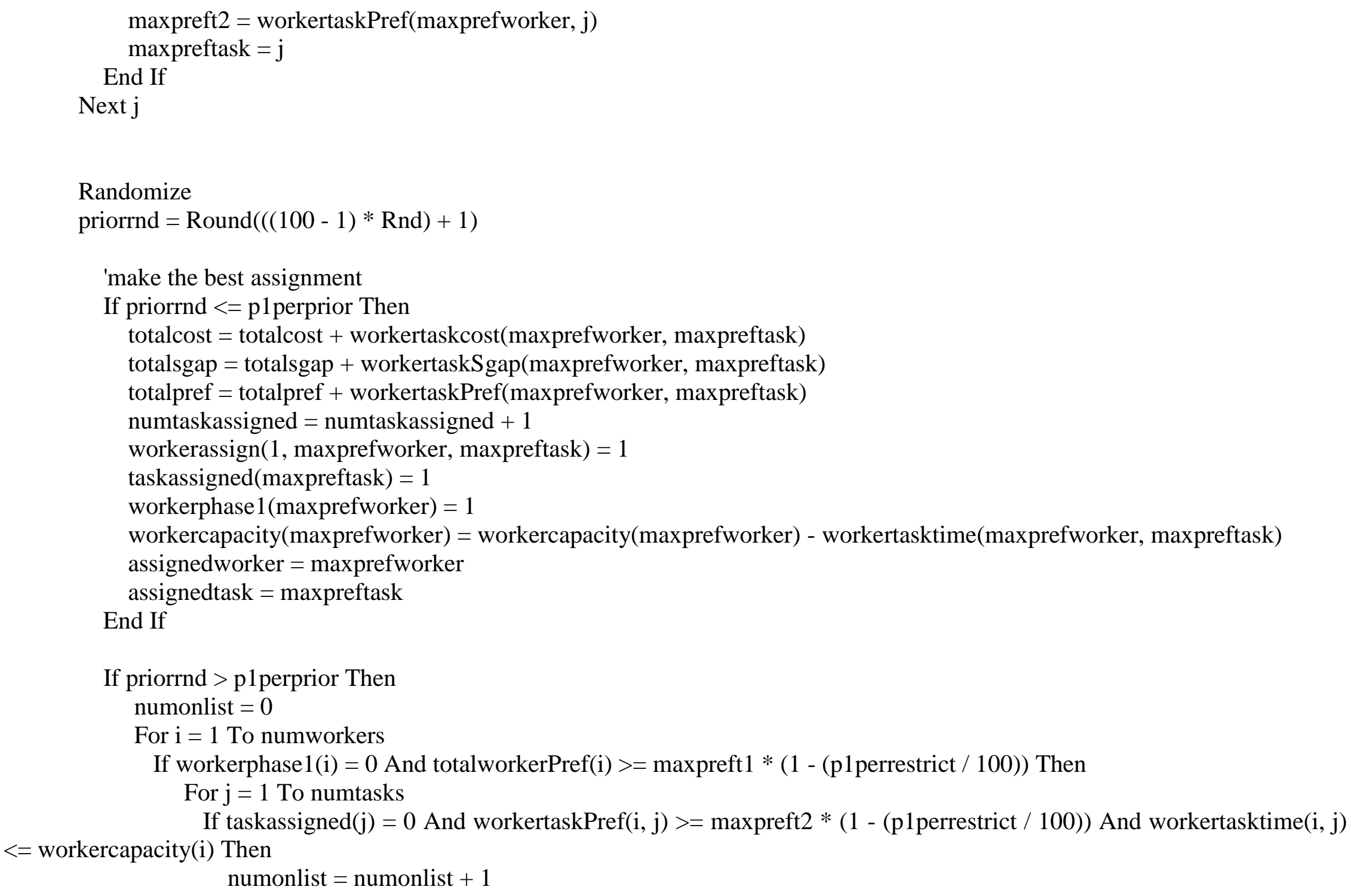


available(numonlist, 1$)=\mathrm{i}$

available(numonlist, 2 ) $=\mathrm{j}$

End If

Next j

End If

Next i

\section{Randomize}

restrictrnd $=\operatorname{Round}((($ numonlist -1$) * \operatorname{Rnd})+1)$

assignedworker $=$ available $($ restrictrnd, 1$)$

assignedtask $=$ available (restrictrnd, 2 )

totalcost $=$ totalcost + workertaskcost $($ assignedworker, assignedtask $)$

totalsgap $=$ totalsgap + workertaskSgap(assignedworker, assignedtask)

totalpref $=$ totalpref + workertaskPref(assignedworker, assignedtask)

numtaskassigned $=$ numtaskassigned +1

workerassign $(1$, assignedworker, assignedtask $)=1$

taskassigned(assignedtask $)=1$

workerphase 1 (assignedworker $)=1$

workercapacity(assignedworker) = workercapacity(assignedworker) - workertasktime(assignedworker, assignedtask) End If

For $\mathrm{k}=1$ To numskills

If workerskill(assignedworker, $\mathrm{k}$ ) < taskskill(assignedtask, $\mathrm{k}$ ) Then workerskill(assignedworker, $\mathrm{k}$ ) = taskskill(assignedtask, $\mathrm{k}$ )

End If

Next k

For $\mathrm{j}=1$ To numtasks 
If taskassigned $(j)=0$ Then

workertaskcost(assignedworker, $\mathrm{j})=0$

workertaskSgap(assignedworker, $\mathrm{j}$ ) $=0$

workertaskPref(assignedworker, $\mathrm{j})=0$

workertasktime (assignedworker, $\mathrm{j})=$ otasktime $(\mathrm{j})$

For $\mathrm{k}=1$ To numskills

If workerskill(assignedworker, $\mathrm{k})<\operatorname{taskskill(j,k)}$ And taskskill(j, $\mathrm{k})>1$ Then

k), taskskill(j, k))

workertaskcost $($ assignedworker, $\mathrm{j})=$ workertaskcost $($ assignedworker, $\mathrm{j})+\operatorname{traincost}(\mathrm{k}$, workerskill(assignedworker,

workertaskSgap(assignedworker, j) $=$ workertaskSgap(assignedworker, j) $\quad+\quad(\operatorname{taskskill}(\mathrm{j}, \quad \mathrm{k}) \quad-$ workerskill(assignedworker, k))

workertaskPref(assignedworker, $\mathrm{j})=$ workertaskPref(assignedworker, $\mathrm{j})+\operatorname{Prefmatrix}(\operatorname{assignedworker,~k)*}$ (taskskill(j, k) - workerskill(assignedworker, k))

workertasktime (assignedworker, $\mathrm{j})=$ workertasktime $($ assignedworker, $\mathrm{j})+$ traintime $(\mathrm{k}$,

workerskill(assignedworker, $\mathrm{k})$, taskskill(j, k))

End If

Next k

End If

Next j

\section{Loop}

End If 'If phase1_on $=1$

'end of phase 1 switch

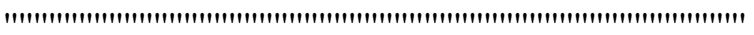

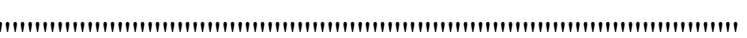

Do While numtaskassigned < numtasks 'repeat until all tasks assigned maxpreft $1=-9999999$ 
For $\mathrm{j}=1$ To numtasks

If taskassigned $(j)=0$ And totaltaskPref $(j)>\operatorname{maxpreft} 1$ Then

maxpreft $1=$ totaltaskPref(j)

maxpreftask $=\mathrm{j}$

End If

Next j

maxpreft $2=-9999999$

maxprefworker $=0$

For $\mathrm{i}=1$ To numworkers

If workertasktime (i, maxpreftask) <= workercapacity(i) And workertaskPref(i, maxpreftask) > maxpreft 2 Then maxpreft $2=$ workertaskPref(i, maxpreftask)

maxprefworker $=\mathrm{i}$

End If

Next i

\section{Randomize}

priorrnd $=\operatorname{Round}(((100-1) *$ Rnd $)+1)$

If maxprefworker $>0$ Then

If priorrnd $<=$ perprior Then

totalcost $=$ totalcost + workertaskcost $($ maxprefworker, maxpreftask $)$

totalsgap $=$ totalsgap + workertaskSgap (maxprefworker, maxpreftask)

totalpref $=$ totalpref + workertaskPref $($ maxprefworker, maxpreftask $)$

numtaskassigned $=$ numtaskassigned +1

workerassign $(1$, maxprefworker, maxpreftask $)=1$

taskassigned $($ maxpreftask $)=1$

workercapacity (maxprefworker) = workercapacity (maxprefworker) - workertasktime (maxprefworker, maxpreftask) assignedworker $=$ maxprefworker 
assignedtask $=$ maxpreftask

End If

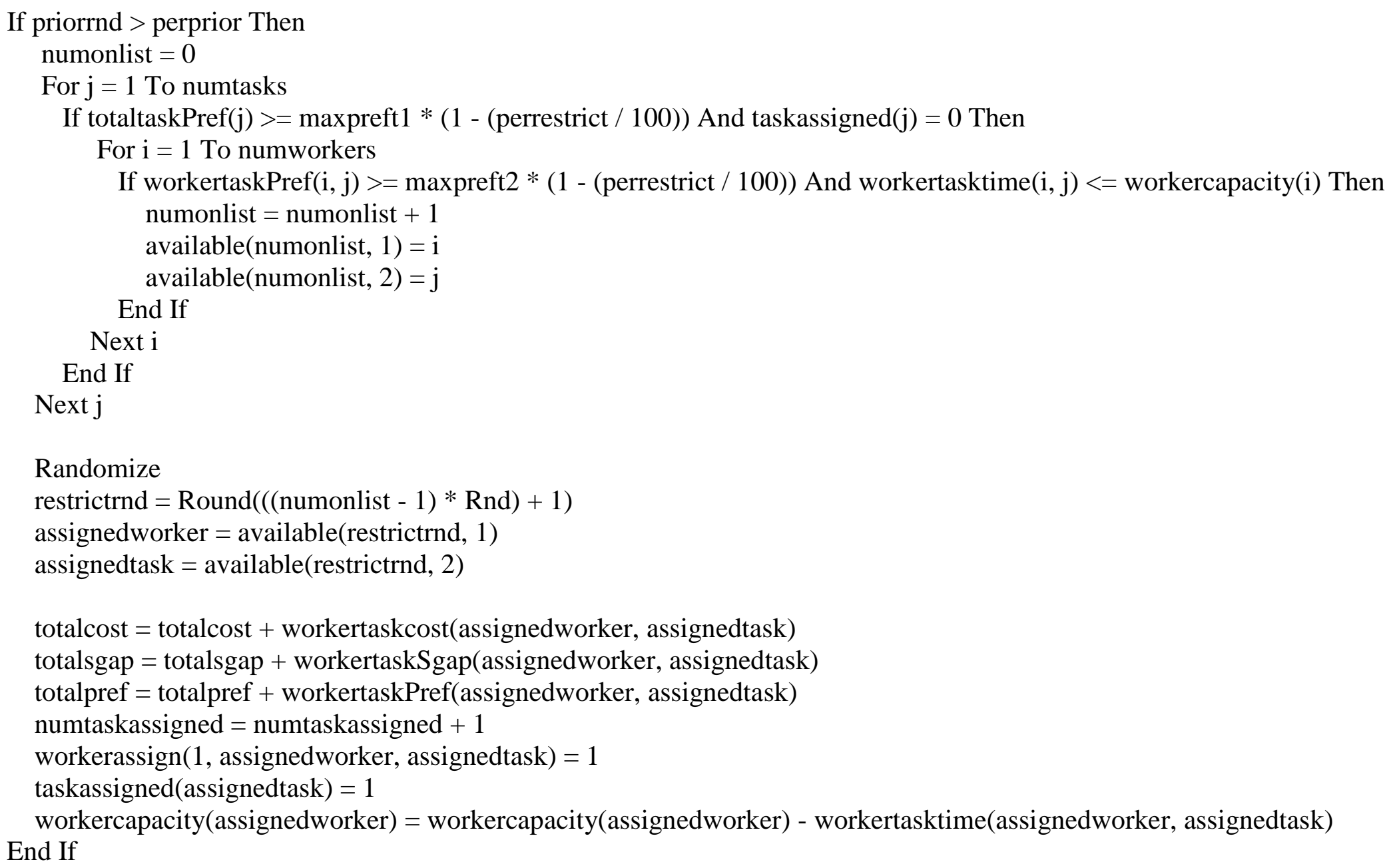


For $\mathrm{k}=1$ To numskills

If workerskill(assignedworker, k) < taskskill(assignedtask, k) Then

workerskill(assignedworker, $\mathrm{k}$ ) = taskskill(assignedtask, $\mathrm{k}$ )

End If

Next k

For $\mathrm{j}=1$ To numtasks

If taskassigned $(j)=0$ Then

workertaskcost $($ assignedworker, $\mathrm{j})=0$

workertaskSgap(assignedworker, $\mathrm{j}$ ) $=0$

workertaskPref(assignedworker, $\mathrm{j}$ ) $=0$

workertasktime(assignedworker, $\mathrm{j})=$ otasktime $(\mathrm{j})$

For $\mathrm{k}=1$ To numskills

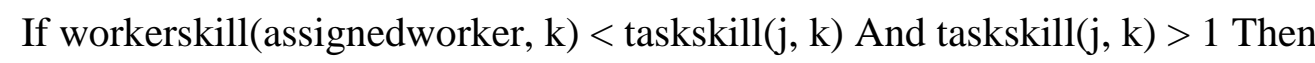

$\mathrm{k})$, taskskill( $(\mathrm{j}, \mathrm{k}))$

workertaskcost(assignedworker, $\mathrm{j})=$ workertaskcost(assignedworker, $\mathrm{j})+\operatorname{traincost}(\mathrm{k}$, workerskill(assignedworker,

workertaskSgap(assignedworker, j) = workertaskSgap(assignedworker, j) $\quad+\quad(\operatorname{taskskill}(\mathrm{j}, \quad \mathrm{k}) \quad-$ workerskill(assignedworker, $\mathrm{k})$ )

workertaskPref(assignedworker, j) = workertaskPref(assignedworker, j) + Prefmatrix(assignedworker, k) * (taskskill(j, k) - workerskill(assignedworker, k))

k), taskskill(j, k))

workertasktime $($ assignedworker, $\mathrm{j})=$ workertasktime $($ assignedworker, $\mathrm{j})+\operatorname{traintime}(\mathrm{k}$, workerskill $($ assignedworker,

$$
\begin{aligned}
& \text { End If } \\
& \text { Next } \mathrm{k} \\
& \text { End If } \\
& \text { Next } \mathrm{j}
\end{aligned}
$$

End If

If maxprefworker $=0$ Then 


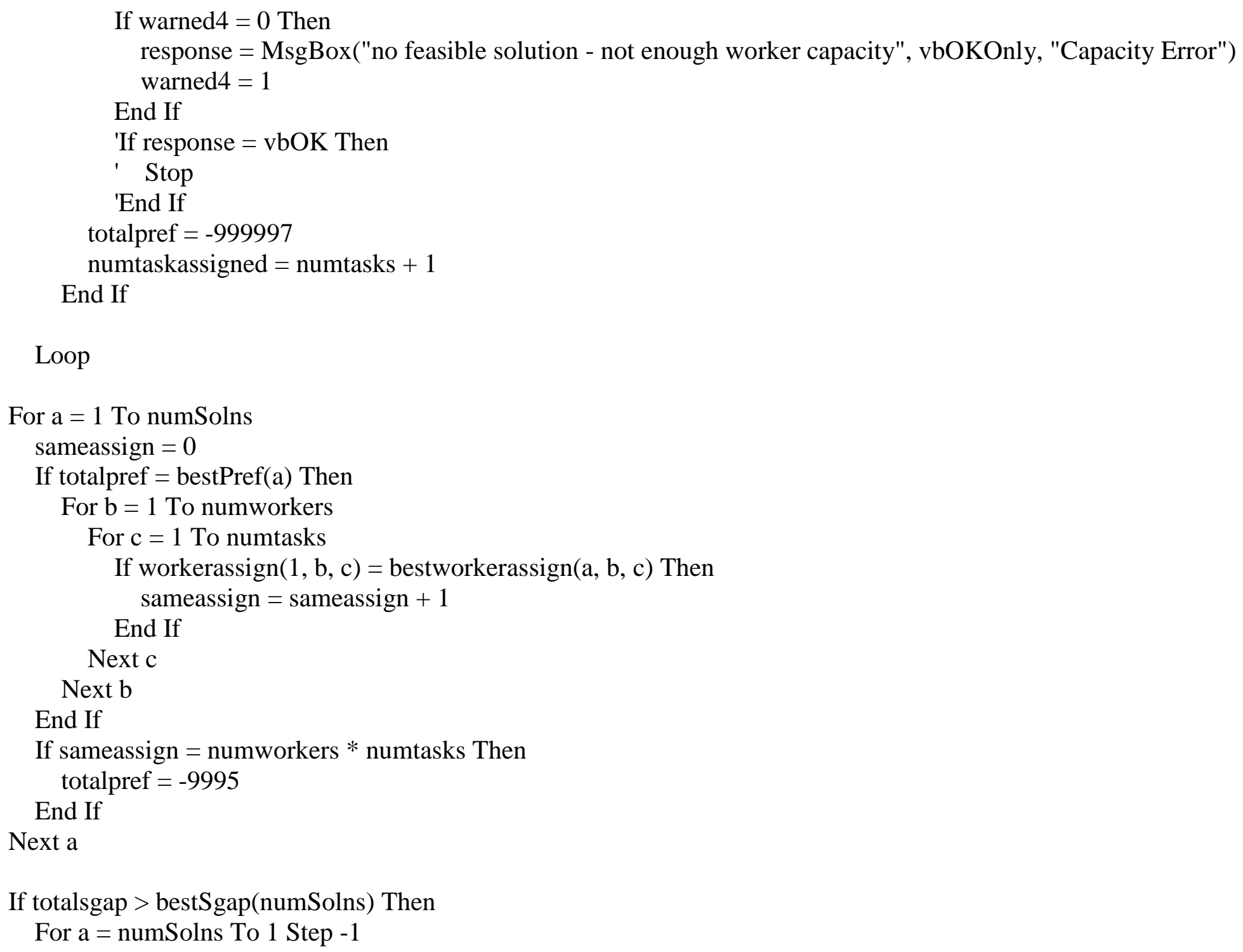




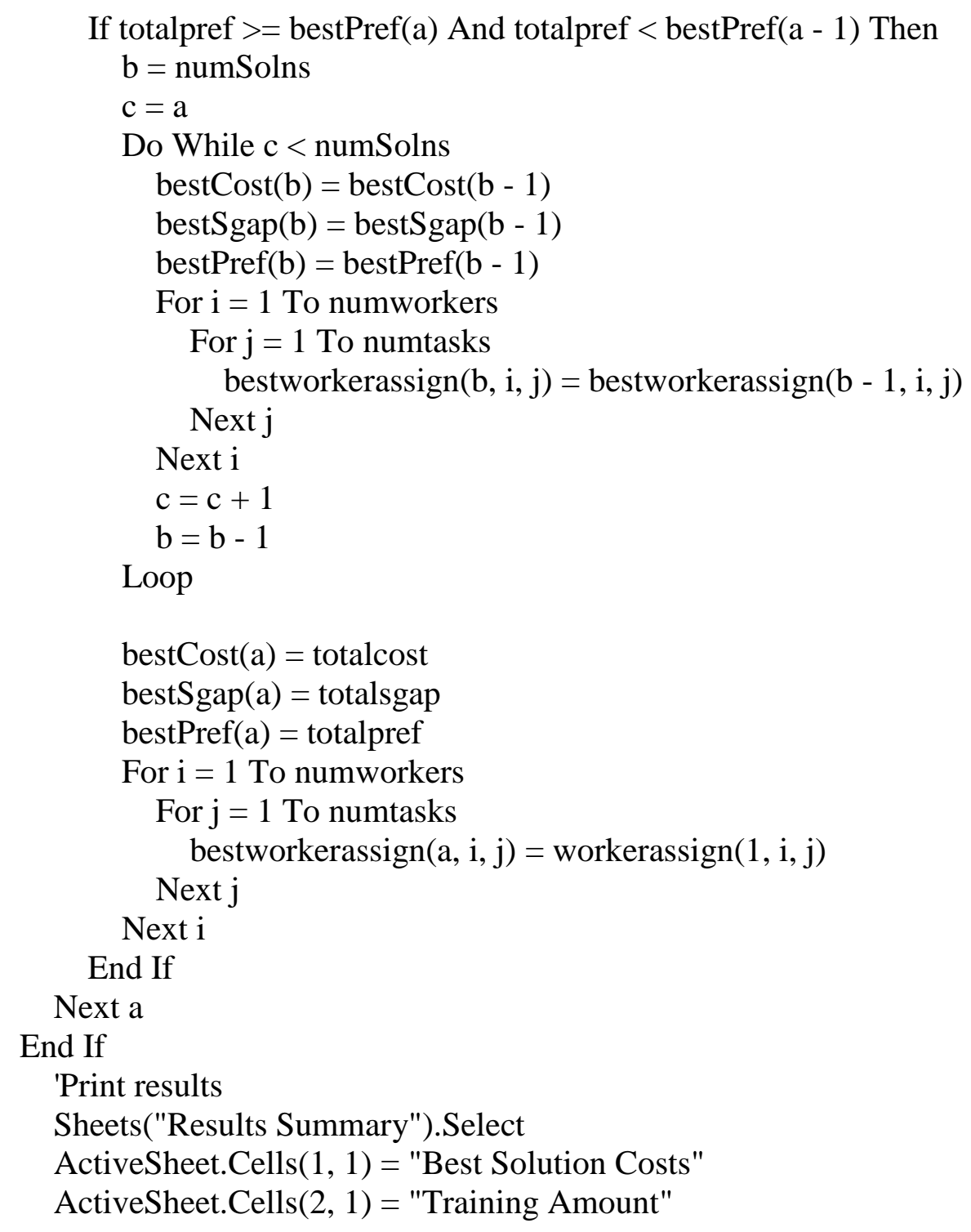


ActiveSheet.Cells $(3,1)=$ "Worker Preference"

For $\mathrm{a}=1$ To numSolns

ActiveSheet.Cells $(1, a+1)=\operatorname{bestCost}(a)$

Next a

For $\mathrm{a}=1$ To numSolns

ActiveSheet.Cells $(2, a+1)=$ bestSgap $(a)$

Next a

For $\mathrm{a}=1$ To numSolns

ActiveSheet.Cells $(3, a+1)=$ bestPref(a)

Next a

Next $r$

endtime $=$ Timer

totaltime $=$ endtime - starttime

Sheets("Results Summary").Select

ActiveSheet.Cells $(3$, numSolns +3$)=$ "Run Time"

ActiveSheet.Cells $(3$, numSolns +4$)=$ totaltime

'Print to Results Summary sheet

Sheets("Results Summary").Select

'ActiveSheet.Cells $(1,1)=$ "Best Solution Costs"

'ActiveSheet.Cells $(1,2)=$ bestsolution $(1)$

ActiveSheet.Cells $(18+2 *$ numtasks, 1) = "Solution \#:"

For $\mathrm{a}=1$ To numSolns

ActiveSheet.Cells $(18+2 *$ numtasks, $2 *$ a $)=2 *$ numSolns + a

Next a

$\mathrm{b}=1$

For $\mathrm{a}=1$ To numSolns $* 2$ 
ActiveSheet.Cells $(19+2 *$ numtasks, a $)=$ "Worker" ActiveSheet.Cells $(19+2 *$ numtasks, $a+1)=$ "Task" cellrow $=20+2 *$ numtasks

For $\mathrm{i}=1$ To numworkers

For $\mathrm{j}=1$ To numtasks

If bestworkerassign $(b, i, j)=1$ Then

ActiveSheet.Cells(cellrow, a) $=\mathrm{i}$

ActiveSheet.Cells (cellrow, $\mathrm{a}+1)=\mathrm{j}$

cellrow $=$ cellrow +1

End If

Next j

Next i

ActiveSheet.Cells $(20+3 *$ numtasks, $a+1)=\operatorname{bestCost}(b)$

ActiveSheet.Cells $(21+3 *$ numtasks, $a+1)=\operatorname{bestSgap}(b)$

ActiveSheet.Cells $(22+3 *$ numtasks, $a+1)=\operatorname{bestPref}(b)$

$\mathrm{b}=\mathrm{b}+1$

$a=a+1$

Next a

ActiveSheet.Cells $(20+3 *$ numtasks, 1$)=$ "Total Cost"

ActiveSheet.Cells $(21+3 *$ numtasks, 1$)=$ "Total Skill Levels"

ActiveSheet.Cells $(22+3 *$ numtasks, 1$)=$ "Total Preference"

\section{End Sub}


'This command button will find the initial solution for Minimum Training Cost

Public Sub Compromise_click()

Dim workerskill() As Single, oworkerskill() As Single

Dim taskskill() As Single, otaskskill() As Single

Dim tasktime() As Single, otasktime() As Single

Dim workercapacity() As Single, oworkercapacity() As Single

Dim traincost() As Single, otraincost() As Single

Dim traintime() As Single, otraintime() As Single

Dim workerassign() As Single

Dim workertaskOFVs() As Single, oworkertaskOFVs() As Single

Dim workertasktime() As Single, oworkertasktime() As Single

Dim Prefmatrix() As Single

Dim taskassigned() As Single

Dim tcost() As Single

Dim ttime() As Single

Dim available() As Single

Dim bestworkerassign() As Single

Dim numworkers As Single, numskills As Single, numtasks As Single, numSolns As Single

Dim totaltaskOFVs() As Single

Dim totalworkerOFVs() As Single

Dim workerphase1() As Single

Dim cellrow As Single

Dim trainingneeds() As Single

Dim skilltrainingneeds() As Single

'everything below is new:

Dim bestOFVs() As Single

Dim TotalOFVs() As Single

Dim st2workerassign() As Single

Dim Valuefn() As Single

Dim Lambda() As Single

Dim varB() As Single 
Dim workerswapped() As Single

Dim baseOF() As Single

Dim tempOFVs() As Single

Dim tempwskill() As Single

Dim tempwtOFVs() As Single

Dim workerswap() As Single

Dim tempworkerassign() As Single

Dim TotalNumObjFn As Single

Dim temptaskcost() As Single

Dim temptaskSgap() As Single

Dim temptaskPref() As Single

Dim possibleswaps() As Single

Dim st2OFV() As Single

TotalNumObjFn $=3$

'Sheets("Results Summary").Cells.Clear

'Sheets("Worker Training Results").Cells.Clear

'Sheets("Skill Training Results").Cells.Clear

'might need to use different parameters here

Sheets("Parameters").Select

p1 perprior $=$ ActiveSheet.Cells $(1,2)$.Value

plperrestrict $=$ ActiveSheet.Cells $(2,2)$. Value

perprior $=$ ActiveSheet.Cells $(3,2)$.Value

perrestrict $=$ ActiveSheet.Cells $(4,2)$.Value

numiter $=$ ActiveSheet.Cells $(5,2)$.Value

phase1_on = 1 'this can be used as a switch to turn phase 1 on or off 
Sheets("Input").Select

numworkers = ActiveSheet.Cells(2, 2). Value

numskills $=$ ActiveSheet.Cells (3, 2).Value

numtasks $=$ ActiveSheet.Cells $(4,2)$.Value

numSolns = ActiveSheet.Cells $(5,2)$.Value

maxcompromises $=50$

'initialize arrays

ReDim workerskill(0 To numworkers $+1,0$ To numskills +1$)$ As Single

ReDim oworkerskill( $(0$ To numworkers $+1,0$ To numskills +1$)$ As Single

ReDim taskskill( 0 To numtasks $+1,0$ To numskills +1$)$ As Single

ReDim otaskskill $(0$ To numtasks $+1,0$ To numskills +1$)$ As Single

ReDim tasktime (0 To numtasks + 1) As Single

ReDim otasktime $(0$ To numtasks +1$)$ As Single

ReDim workercapacity (0 To numworkers + 1) As Single

ReDim oworkercapacity (0 To numworkers +1$)$ As Single

ReDim traincost $(0$ To numskills $+1,0$ To 5, 0 To 5) As Single

ReDim otraincost( 0 To numskills $+1,0$ To 5, 0 To 5) As Single

ReDim traintime(0 To numskills $+1,0$ To 5, 0 To 5) As Single

ReDim otraintime( 0 To numskills $+1,0$ To 5, 0 To 5) As Single

'added a dimension to the two matrices below

ReDim workerassign $(0$ To 1, 0 To 1, 0 To numworkers $+1,0$ To numtasks +1$)$ As Single

ReDim bestworkerassign $(0$ To TotalNumObjFn $+1,0$ To numSolns $+1,0$ To numworkers $+1,0$ To numtasks +1$)$ As Single

ReDim workertaskOFVs $(0$ To TotalNumObjFn $+1,0$ To numworkers $+1,0$ To numtasks +1$)$ As Single

ReDim oworkertaskOFVs(0 To TotalNumObjFn $+1,0$ To numworkers $+1,0$ To numtasks +1$)$ As Single

ReDim Prefmatrix $(0$ To numworkers $+1,0$ To numskills +1$)$ As Single

ReDim workertasktime $(0$ To numworkers $+1,0$ To numtasks +1$)$ As Single

ReDim oworkertasktime $(0$ To numworkers $+1,0$ To numtasks +1$)$ As Single

ReDim taskassigned(0 To numtasks +1 ) As Single 
ReDim tcost $(0$ To numskills $+1,0$ To 5$)$ As Single

ReDim ttime( 0 To numskills $+1,0$ To 5) As Single

ReDim available (0 To numworkers * numtasks $+1,0$ To 3$)$ As Single

ReDim totaltaskOFVs $(0$ To TotalNumObjFn $+1,0$ To numtasks +1$)$ As Single

ReDim totalworkerOFVs(0 To TotalNumObjFn $+1,0$ To numworkers + 1) As Single

ReDim workerphase1(0 To numworkers +1$)$ As Single

ReDim trainingneeds $(0$ To numworkers $+1,0$ To numskills +1$)$ As Single

ReDim skilltrainingneeds(0 To numskills $+1,0$ To 5) As Single

'new one below:

ReDim bestOFVs(0 To TotalNumObjFn + 1, 0 To TotalNumObjFn + 1, 0 To numSolns + 1) As Single

ReDim TotalOFVs $(0$ To TotalNumObjFn +1$)$ As Single

ReDim possibleswaps $(0$ To numworkers $+1,0$ To numtasks $+1,0$ To numworkers $+1,0$ To numtasks +1$)$ As Single

ReDim st2workerassign $(0$ To maxcompromises $+1,0$ To numworkers +1 , 0 To numtasks +1$)$ As Single

ReDim Valuefn $(0$ To maxcompromises +1$)$ As Single

ReDim Lambda(0 To TotalNumObjFn + 1, 0 To maxcompromises + 1) As Single

ReDim varB(0 To TotalNumObjFn + 1, 0 To maxcompromises + 1) As Single

ReDim baseOF(0 To maxcompromises + 1) As Single

ReDim workerswapped $(0$ To numworkers +1$)$ As Single

ReDim tempOFVs(0 To TotalNumObjFn +1$)$ As Single

ReDim tempwskill $(0$ To numworkers $+1,0$ To numskills +1$)$ As Single

ReDim tempwtOFVs $(0$ To TotalNumObjFn $+1,0$ To numworkers $+1,0$ To numtasks +1$)$ As Single

ReDim workerswap $(0$ To maxcompromises $+1,0$ To numworkers +1$)$ As Single

ReDim tempworkerassign( 0 To 1, 0 To numworkers $+1,0$ To numtasks +1$)$ As Single

ReDim temptaskcost $(0$ To numworkers $+1,0$ To numtasks +1$)$ As Single

ReDim temptaskSgap $(0$ To numworkers $+1,0$ To numtasks +1$)$ As Single

ReDim temptaskPref $(0$ To numworkers $+1,0$ To numtasks +1$)$ As Single

ReDim possibleswaps(0 To numworkers $+1,0$ To numtasks $+1,0$ To numworkers $+1,0$ To numtasks $+1,0$ To TotalNumObjFn + 1) As Single

ReDim st2OFV(0 To maxcompromises + 1, 0 To TotalNumObjFn + 1) As Single

starttime $=$ Timer 
'new thing below

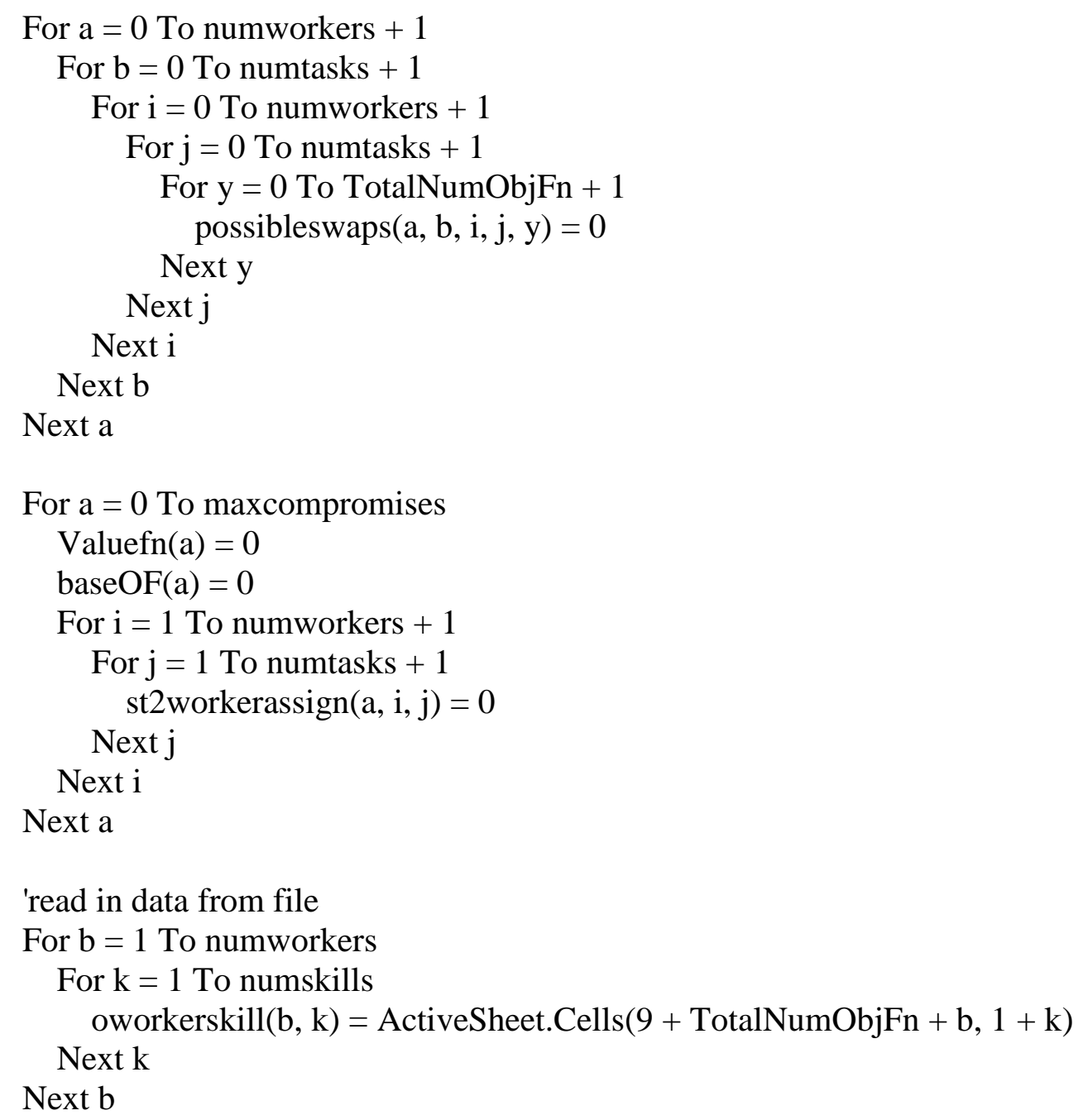




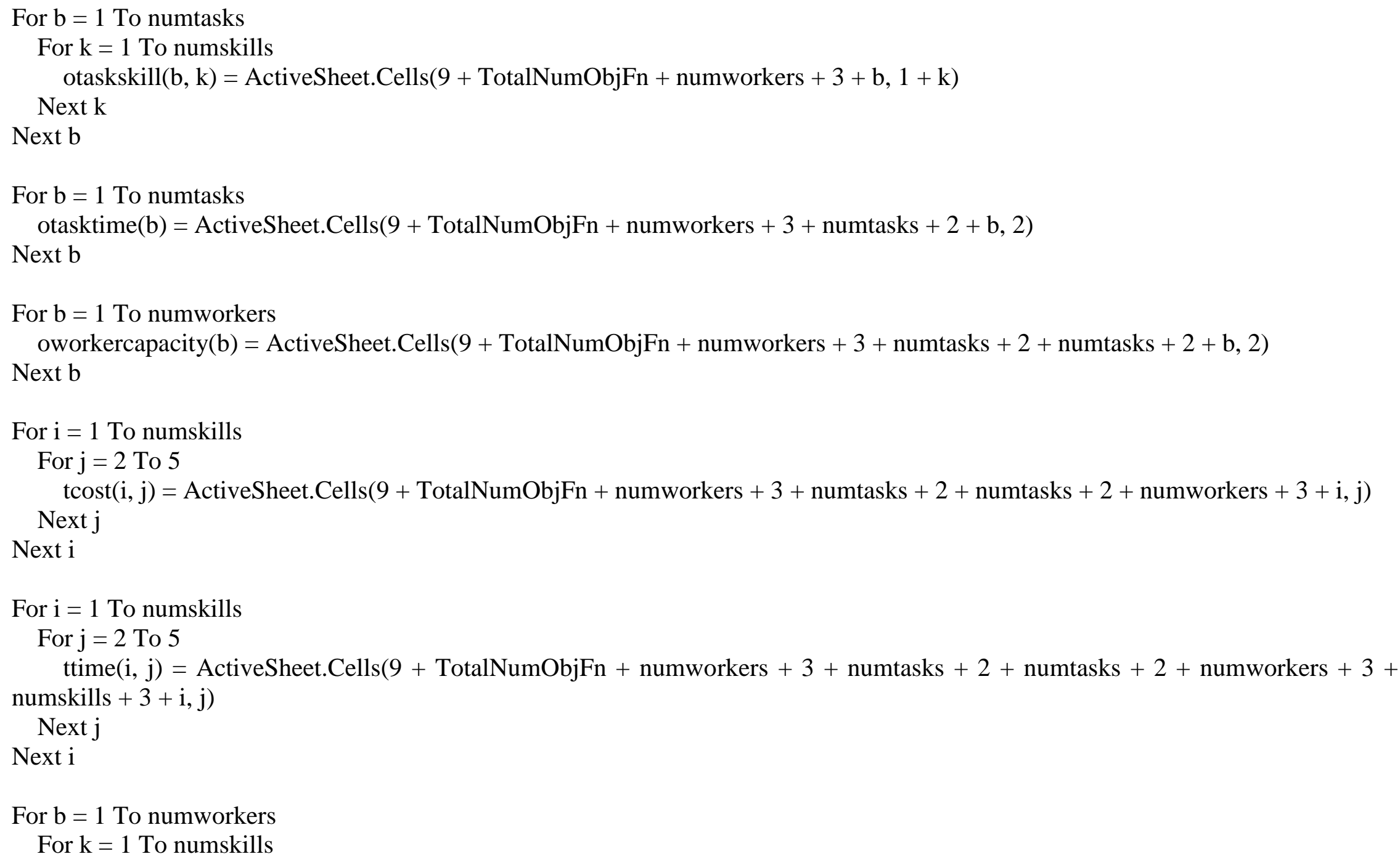


Prefmatrix $(\mathrm{b}, \mathrm{k})=$ ActiveSheet.Cells $(9+$ TotalNumObjFn + numworkers $+3+$ numtasks $+2+$ numtasks $+2+$ numworkers +3 + numskills $+3+$ numskills $+3+\mathrm{b}, \mathrm{k}+1$ )

Next k

Next b

'done reading in values from the excel sheet

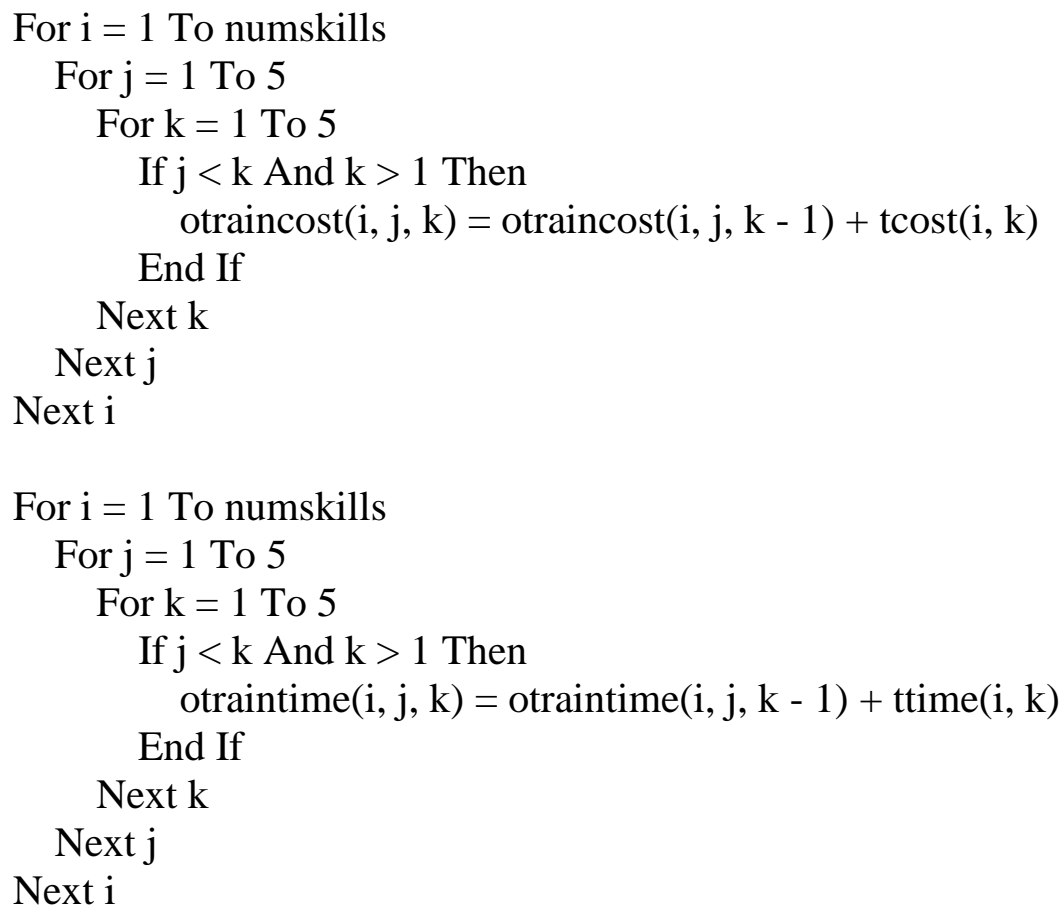


Sheets("Results Summary").Select

For a $=1$ To numSolns

ActiveSheet.Cells $(1, \mathrm{a}+1)=$ ActiveSheet.Cells $(8+\operatorname{numtasks}, 2 *$ a)

Next a

For $\mathrm{a}=1$ To numSolns

ActiveSheet.Cells $(2, a+1)=$ ActiveSheet.Cells $(15+2 *$ numtasks, $2 *$ a)

Next a

For $\mathrm{a}=1$ To numSolns

ActiveSheet.Cells $(3, \mathrm{a}+1)=\operatorname{ActiveSheet} . \operatorname{Cells}(22+3 *$ numtasks, $2 *$ a $)$

Next a

st 2 count $=1$

prefsoln = Application.InputBox("What is the preferred solution so far?", "")

UserForm1.Label1.Caption = "To what percent would you like to compromise each objective? "

$\operatorname{varB}(1$, st 2 count $)=0$

$\operatorname{varB}(2$, st 2 count $)=0$

$\operatorname{varB}(3$, st 2 count $)=0$

If prefsoln $<=$ numSolns Then

cellrow $=7$

cellcol $=$ prefsoln $* 2$

Do Until baseOF(st2count $)=2$ Or baseOF $($ st 2 count $)=3$

baseOF(st2count) = InputBox("Which objective do you want to improve? (2-Training Amount, or 3-Worker Preference)", "') Loop

totalcost $=$ ActiveSheet Cells $($ cellrow + numtasks +1 , cellcol $)$

totalsgap $=$ ActiveSheet .Cells $($ cellrow + numtasks +2 , cellcol $)$

totalpref $=$ ActiveSheet .Cells $($ cellrow + numtasks +3 , cellcol $)$

If baseOF( $\mathrm{st} 2$ count $)=2$ Then 
'UserForm1.Label1.Caption = UserForm1.Label1.Caption \& baseOF(st2count) \& " from " \& totalsgap \& " to " \& totalsgap * 1.1 \& "(10\%)"

UserForm1.Frame1.Caption $=$ "Total Cost (Obj 1)"

UserForm1.Frame2.Caption = "Worker Preference $(\mathrm{Obj} 3) "$

UserForm1.OptionButton1.Caption $=$ totalcost $* 0.04 \& "(4 \%) "$

UserForm1.OptionButton2.Caption $=$ totalcost $* 0.07 \& "(7 \%) "$

UserForm1.OptionButton3.Caption $=$ totalcost $* 0.1 \& "(10 \%) "$

UserForm1.OptionButton4.Caption $=$ totalcost $* 0.13 \&$ " $(13 \%) "$

UserForm1.OptionButton5.Caption $=$ totalcost $* 0.16 \& "(16 \%) "$

UserForm1.OptionButton6.Caption $=$ totalpref $* 0.04 \& "(4 \%) "$

UserForm1.OptionButton7.Caption $=$ totalpref $* 0.07 \& "(7 \%) "$

UserForm1.OptionButton8.Caption $=$ totalpref $* 0.1 \& "(10 \%) "$

UserForm1.OptionButton9.Caption $=$ totalpref $* 0.13 \&$ \& $(13 \%) "$

UserForm1.OptionButton10.Caption $=$ totalpref $* 0.16 \& "(16 \%) "$

UserForm1.Show

If $($ UserForm1.OptionButton1. Value $=$ True $)$ Then $\operatorname{varB}(1$, st 2 count $)=0.04$

If $($ UserForm1.OptionButton2. Value $=$ True $)$ Then $\operatorname{varB}(1$, st2count $)=0.07$

If $($ UserForm1.OptionButton3. Value $=$ True $)$ Then $\operatorname{varB}(1$, st 2 count $)=0.1$

If $($ UserForm1.OptionButton4. Value $=$ True $)$ Then $\operatorname{varB}(1$, st 2 count $)=0.13$

If $($ UserForm1.OptionButton5.Value $=$ True $)$ Then $\operatorname{varB}(1$, st 2 count $)=0.16$

If $($ UserForm1.OptionButton6. Value $=$ True $)$ Then $\operatorname{varB}(3$, st 2 count $)=0.04$

If $($ UserForm1.OptionButton7.Value $=$ True $)$ Then $\operatorname{varB}(3$, st 2 count $)=0.07$

If $($ UserForm1.OptionButton8.Value $=$ True $)$ Then $\operatorname{varB}(3$, st2count $)=0.1$

If $($ UserForm1.OptionButton9. Value $=$ True $)$ Then $\operatorname{varB}(3$, st 2 count $)=0.13$

If $($ UserForm1.OptionButton10.Value $=$ True $)$ Then $\operatorname{varB}(3$, st2count $)=0.16$

End If

If baseOF( st2count $)=3$ Then

'UserForm1.Label1.Caption = UserForm1.Label1.Caption \& baseOF(st2count) \& " from " \& totalpref \& " to " \& totalpref * 1.1 $\&$ "(10\%)"

UserForm1.Frame1.Caption = "Total Cost (Obj 1)" 
UserForm1.Frame2.Caption = "Training Amount (Obj 2)"

UserForm1.OptionButton1.Caption = totalcost $* 0.04 \&$ \& $(4 \%) "$

UserForm1.OptionButton2.Caption $=$ totalcost $* 0.07 \&$ " $(7 \%) "$

UserForm1.OptionButton3.Caption $=$ totalcost $* 0.1 \&$ " $(10 \%) "$

UserForm1.OptionButton4.Caption $=$ totalcost $* 0.13 \&$ " $(13 \%)$ "

UserForm1.OptionButton5.Caption $=$ totalcost $* 0.16 \& "(16 \%) "$

UserForm1.OptionButton6.Caption = totalsgap * $0.04 \&$ \& $(4 \%) "$

UserForm1.OptionButton7.Caption = totalsgap $* 0.07 \&$ " $(7 \%) "$

UserForm1.OptionButton8.Caption = totalsgap * $0.1 \&$ " $(10 \%) "$

UserForm1.OptionButton9.Caption $=$ totalsgap * $0.13 \&$ " $(13 \%) "$

UserForm1.OptionButton10.Caption = totalsgap * $0.16 \&$ " $(16 \%) "$

UserForm1.Show

If $($ UserForm1. OptionButton1. Value $=$ True $)$ Then $\operatorname{varB}(1$, st 2 count $)=0.04$

If $($ UserForm1.OptionButton2. Value $=$ True $)$ Then $\operatorname{varB}(1$, st2count $)=0.07$

If $($ UserForm1.OptionButton3. Value $=$ True $)$ Then $\operatorname{varB}(1$, st2count $)=0.1$

If $($ UserForm1.OptionButton4. Value $=$ True $)$ Then $\operatorname{varB}(1$, st 2 count $)=0.13$

If $($ UserForm1.OptionButton5. Value $=$ True $)$ Then $\operatorname{varB}(1$, st2count $)=0.16$

If $($ UserForm1.OptionButton6. Value $=$ True $)$ Then $\operatorname{varB}(2$, st 2 count $)=0.04$

If $($ UserForm1.OptionButton7.Value $=$ True $)$ Then $\operatorname{varB}(2$, st 2 count $)=0.07$

If $($ UserForm1.OptionButton8.Value $=$ True $)$ Then $\operatorname{varB}(2$, st2count $)=0.1$

If $($ UserForm1.OptionButton9. Value $=$ True $)$ Then $\operatorname{varB}(2$, st 2 count $)=0.13$

If $($ UserForm1.OptionButton10. Value $=$ True $)$ Then $\operatorname{varB}(2$, st 2 count $)=0.16$

End If

End If

If prefsoln $>$ numSolns And prefsoln $<=$ numSolns $* 2$ Then

cellrow $=7+$ numtasks +6

cellcol $=($ prefsoln - numSolns $) * 2$

Do Until baseOF(st 2 count $)=1$ Or baseOF $(\operatorname{st} 2$ count $)=3$

baseOF(st2count) = InputBox("Which objective do you want to improve? (1-Training Cost, or 3-Worker Preference)", "") Loop 
totalcost $=$ ActiveSheet Cells $($ cellrow + numtasks +1 , cellcol $)$

totalsgap $=$ ActiveSheet Cells $($ cellrow + numtasks +2 , cellcol $)$

totalpref $=$ ActiveSheet.Cells (cellrow + numtasks +3 , cellcol $)$

If baseOF( st 2 count $)=1$ Then

'UserForm1.Label1.Caption = UserForm1.Label1.Caption \& baseOF(st2count) \& " from " \& totalcost \& " to " \& totalcost * 0.9 $\&$ " $(10 \%) "$

UserForm1.Frame1.Caption = "Training Amount (Obj 2)"

UserForm1.Frame2.Caption = "Worker Preference $(\mathrm{Obj} 3) "$

UserForm1.OptionButton1.Caption = totalsgap * $0.04 \&$ \& $(4 \%) "$

UserForm1.OptionButton2.Caption = totalsgap * $0.07 \& "(7 \%) "$

UserForm1.OptionButton3.Caption $=$ totalsgap $* 0.1 \& "(10 \%) "$

UserForm1.OptionButton4.Caption = totalsgap * $0.13 \&$ " (13\%)"

UserForm1.OptionButton5.Caption $=$ totalsgap $* 0.16 \&$ " $(16 \%) "$

UserForm1.OptionButton6.Caption $=$ totalpref $* 0.04 \&$ " $(4 \%) "$

UserForm1.OptionButton7.Caption $=$ totalpref $* 0.07 \&$ \& $(7 \%) "$

UserForm1.OptionButton8.Caption $=$ totalpref $* 0.1 \& "(10 \%) "$

UserForm1.OptionButton9.Caption $=$ totalpref $* 0.13 \&$ " $(13 \%) "$

UserForm1.OptionButton10.Caption = totalpref $* 0.16 \& "(16 \%) "$

UserForm1.Show

If $($ UserForm1.OptionButton1. Value $=$ True $)$ Then $\operatorname{varB}(2$, st 2 count $)=0.04$

If $($ UserForm1.OptionButton2. Value $=$ True $)$ Then $\operatorname{varB}(2$, st 2 count $)=0.07$

If $($ UserForm1.OptionButton3.Value $=$ True $)$ Then $\operatorname{varB}(2$, st2count $)=0.1$

If $($ UserForm1.OptionButton4.Value $=$ True $)$ Then $\operatorname{varB}(2$, st2count $)=0.13$

If $($ UserForm1.OptionButton5. Value $=$ True $)$ Then $\operatorname{varB}(2$, st 2 count $)=0.16$

If $($ UserForm1.OptionButton6.Value $=$ True $)$ Then $\operatorname{varB}(3$, st 2 count $)=0.04$

If $($ UserForm1.OptionButton7. Value $=$ True $)$ Then $\operatorname{varB}(3$, st 2 count $)=0.07$

If $($ UserForm1.OptionButton8.Value $=$ True $)$ Then $\operatorname{varB}(3$, st 2 count $)=0.1$

If $($ UserForm1.OptionButton9. Value $=$ True $)$ Then $\operatorname{varB}(3, \mathrm{st} 2$ count $)=0.13$

If $($ UserForm1.OptionButton10.Value $=$ True $)$ Then $\operatorname{varB}(3$, st2 count $)=0.16$

End If 
If baseOF(st2count) $=3$ Then

'UserForm1.Label1.Caption = UserForm1.Label1.Caption \& baseOF(st2count) \& " from " \& totalpref \& " to " \& totalpref $* 1.1$ $\&$ "(10\%)"

UserForm1.Frame1.Caption = "Total Cost $(\mathrm{Obj} 1) "$

UserForm1.Frame2.Caption = "Training Amount (Obj 2)"

UserForm1.OptionButton1.Caption $=$ totalcost $* 0.04 \&$ " $(4 \%) "$

UserForm1.OptionButton2.Caption $=$ totalcost $* 0.07 \&$ " $(7 \%) "$

UserForm1.OptionButton3.Caption $=$ totalcost $* 0.1 \&$ " $(10 \%) "$

UserForm1.OptionButton4.Caption $=$ totalcost $* 0.13 \&$ " $(13 \%) "$

UserForm1.OptionButton5.Caption $=$ totalcost $* 0.16 \&$ " $(16 \%) "$

UserForm1.OptionButton6.Caption $=$ totalsgap $* 0.04 \& "(4 \%) "$

UserForm1.OptionButton7.Caption $=$ totalsgap $* 0.07 \& "(7 \%) "$

UserForm1.OptionButton8.Caption = totalsgap * $0.1 \&$ " $(10 \%) "$

UserForm1.OptionButton9.Caption = totalsgap * $0.13 \& "(13 \%) "$

UserForm1.OptionButton10.Caption = totalsgap * $0.16 \& "(16 \%) "$

UserForm1.Show

If $($ UserForm1.OptionButton 1. Value $=$ True $)$ Then $\operatorname{varB}(1, \mathrm{st} 2$ count $)=0.04$

If $($ UserForm1.OptionButton2. Value $=$ True $)$ Then $\operatorname{varB}(1$, st 2 count $)=0.07$

If $($ UserForm1.OptionButton3. Value $=$ True $)$ Then $\operatorname{varB}(1$, st 2 count $)=0.1$

If $($ UserForm1.OptionButton4.Value $=$ True $)$ Then $\operatorname{varB}(1$, st 2 count $)=0.13$

If $($ UserForm1.OptionButton5.Value $=$ True $)$ Then $\operatorname{varB}(1, \mathrm{st} 2$ count $)=0.16$

If $($ UserForm1.OptionButton6. Value $=$ True $)$ Then $\operatorname{varB}(2$, st 2 count $)=0.04$

If $($ UserForm1.OptionButton7.Value $=$ True $)$ Then $\operatorname{varB}(2$, st 2 count $)=0.07$

If $($ UserForm1.OptionButton8.Value $=$ True $)$ Then $\operatorname{varB}(2$, st 2 count $)=0.1$

If $($ UserForm1.OptionButton9. Value $=$ True $)$ Then $\operatorname{varB}(2$, st2count $)=0.13$

If $($ UserForm1.OptionButton10. Value $=$ True $)$ Then $\operatorname{varB}(2$, st2count $)=0.16$

\section{End If}

End If

If prefsoln $>$ numSolns $* 2$ And prefsoln $<=$ numSolns $* 3$ Then

cellrow $=7+$ numtasks $+6+$ numtasks +6

cellcol $=($ prefsoln $-2 *$ numSolns $) * 2$ 
Do Until baseOF(st2count $)=2$ Or baseOF(st 2 count $)=1$

baseOF(st2count) = InputBox("Which objective do you want to improve? (1-Training Cost, or 2-Training Amount)", "") Loop

totalcost $=$ ActiveSheet.Cells $($ cellrow + numtasks +1 , cellcol $)$

totalsgap $=$ ActiveSheet Cells $($ cellrow + numtasks +2 , cellcol $)$

totalpref $=$ ActiveSheet.Cells (cellrow + numtasks +3 , cellcol $)$

If baseOF( st2count $)=1$ Then

'UserForm1.Label1.Caption = UserForm1.Label1.Caption \& baseOF(st2count) \& " from " \& totalcost \& " to " \& totalcost * 0.9 $\&$ "(10\%)"

UserForm1.Frame1.Caption = "Training Amount (Obj 2)"

UserForm1.Frame2.Caption = "Worker Preference $($ Obj 3)"

UserForm1.OptionButton1.Caption = totalsgap * $0.04 \& "(4 \%) "$

UserForm1.OptionButton2.Caption = totalsgap * $0.07 \& "(7 \%) "$

UserForm1.OptionButton3.Caption $=$ totalsgap $* 0.1 \&$ " $(10 \%) "$

UserForm1.OptionButton4.Caption = totalsgap * $0.13 \&$ " $(13 \%) "$

UserForm1.OptionButton5.Caption $=$ totalsgap $* 0.16 \& "(16 \%) "$

UserForm1.OptionButton6.Caption $=$ totalpref $* 0.04 \& "(4 \%) "$

UserForm 1.OptionButton7.Caption $=$ totalpref $* 0.07 \&$ " $(7 \%) "$

UserForm1.OptionButton8.Caption $=$ totalpref $* 0.1 \& "(10 \%) "$

UserForm1.OptionButton9.Caption = totalpref * $0.13 \&$ " $(13 \%) "$

UserForm1.OptionButton10.Caption = totalpref $* 0.16 \&$ " $(16 \%) "$

UserForm1.Show

If $($ UserForm1.OptionButton 1 .Value $=$ True $)$ Then $\operatorname{varB}(2$, st 2 count $)=0.04$

If $($ UserForm1.OptionButton2. Value $=$ True $)$ Then $\operatorname{varB}(2$, st2count $)=0.07$

If $($ UserForm1.OptionButton3.Value $=$ True $)$ Then $\operatorname{varB}(2$, st 2 count $)=0.1$

If $($ UserForm1.OptionButton4. Value $=$ True $)$ Then $\operatorname{varB}(2$, st 2 count $)=0.13$

If $($ UserForm1.OptionButton5. Value $=$ True $)$ Then $\operatorname{varB}(2$, st 2 count $)=0.16$

If $($ UserForm1.OptionButton6.Value $=$ True $)$ Then $\operatorname{varB}(3$, st 2 count $)=0.04$

If $($ UserForm1.OptionButton7.Value $=$ True $)$ Then $\operatorname{varB}(3$, st 2 count $)=0.07$

If $($ UserForm1.OptionButton8.Value $=$ True $)$ Then $\operatorname{varB}(3$, st 2 count $)=0.1$ 
If $($ UserForm1.OptionButton9. Value $=$ True $)$ Then $\operatorname{varB}(3$, st2count $)=0.13$

If $($ UserForm1.OptionButton10. Value $=$ True $)$ Then $\operatorname{varB}(3$, st2count $)=0.16$

End If

If baseOF(st2count $)=2$ Then

'UserForm1.Label1.Caption = UserForm1.Label1.Caption \& baseOF(st2count) \& " from " \& totalsgap \& " to " \& totalsgap * 1.1 $\&$ "(10\%)"

UserForm1.Frame1.Caption $=$ "Total Cost $($ Obj 1)"

UserForm1.Frame2.Caption = "Worker Preference $(\mathrm{Obj} 3) "$

UserForm1.OptionButton1.Caption $=$ totalcost $* 0.04 \& "(4 \%) "$

UserForm1.OptionButton2. Caption $=$ totalcost $* 0.07 \&$ " $(7 \%) "$

UserForm1.OptionButton3.Caption $=$ totalcost $* 0.1 \& "(10 \%) "$

UserForm1.OptionButton4.Caption $=$ totalcost $* 0.13 \&$ " $(13 \%) "$

UserForm1.OptionButton5.Caption $=$ totalcost $* 0.16 \&$ " $(16 \%) "$

UserForm1.OptionButton6.Caption $=$ totalpref $* 0.04 \&$ " $(4 \%) "$

UserForm1.OptionButton7.Caption $=$ totalpref $* 0.07 \&$ " $(7 \%) "$

UserForm1.OptionButton8.Caption = totalpref $* 0.1 \&$ " $(10 \%) "$

UserForm1.OptionButton9.Caption $=$ totalpref $* 0.13 \&$ " $(13 \%) "$

UserForm1.OptionButton10.Caption = totalpref $* 0.16 \&$ " $(16 \%) "$

UserForm1.Show

If $($ UserForm1.OptionButton1. Value $=$ True $)$ Then $\operatorname{varB}(1$, st2count $)=0.04$

If $($ UserForm1.OptionButton2. Value $=$ True $)$ Then $\operatorname{varB}(1$, st 2 count $)=0.07$

If $($ UserForm1.OptionButton3.Value $=$ True $)$ Then $\operatorname{varB}(1$, st2count $)=0.1$

If $($ UserForm1.OptionButton4. Value $=$ True $)$ Then $\operatorname{varB}(1$, st 2 count $)=0.13$

If $($ UserForm1.OptionButton5. Value $=$ True $)$ Then $\operatorname{varB}(1, \mathrm{st} 2$ count $)=0.16$

If $($ UserForm1.OptionButton6.Value $=$ True $)$ Then $\operatorname{varB}(3$, st 2 count $)=0.04$

If $($ UserForm1.OptionButton7.Value $=$ True $)$ Then $\operatorname{varB}(3$, st 2 count $)=0.07$

If $($ UserForm1.OptionButton8.Value $=$ True $)$ Then $\operatorname{varB}(3$, st2count $)=0.1$

If $($ UserForm1.OptionButton9. Value $=$ True $)$ Then $\operatorname{varB}(3$, st 2 count $)=0.13$

End If

If $($ UserForm1.OptionButton10.Value $=$ True $)$ Then $\operatorname{varB}(3$, st 2 count $)=0.16$

End If 


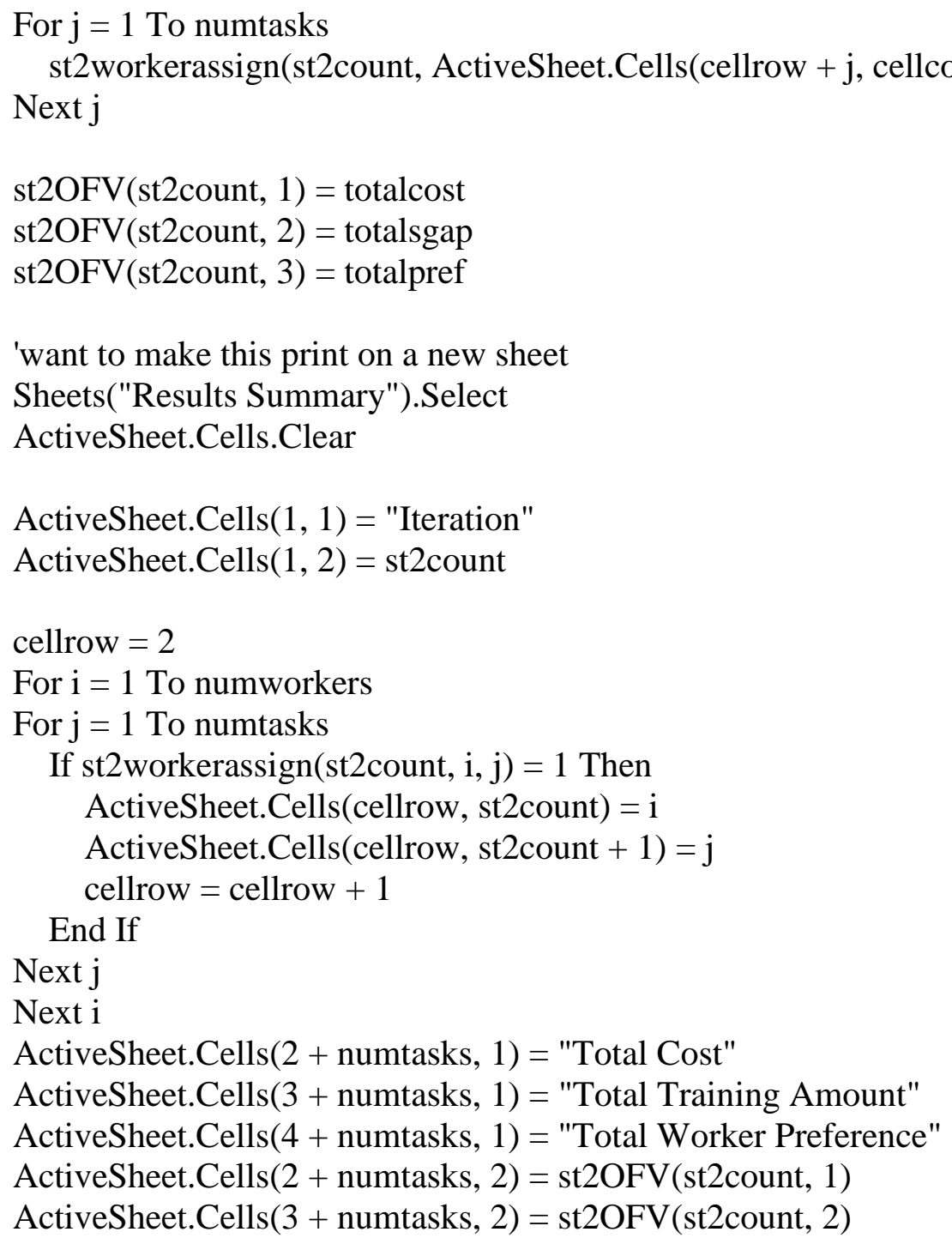


ActiveSheet.Cells $(4+$ numtasks, 2$)=\operatorname{st} 2 \mathrm{OFV}($ st2count, 3$)$

usersatisfied $=0$

Do While usersatisfied $=0$

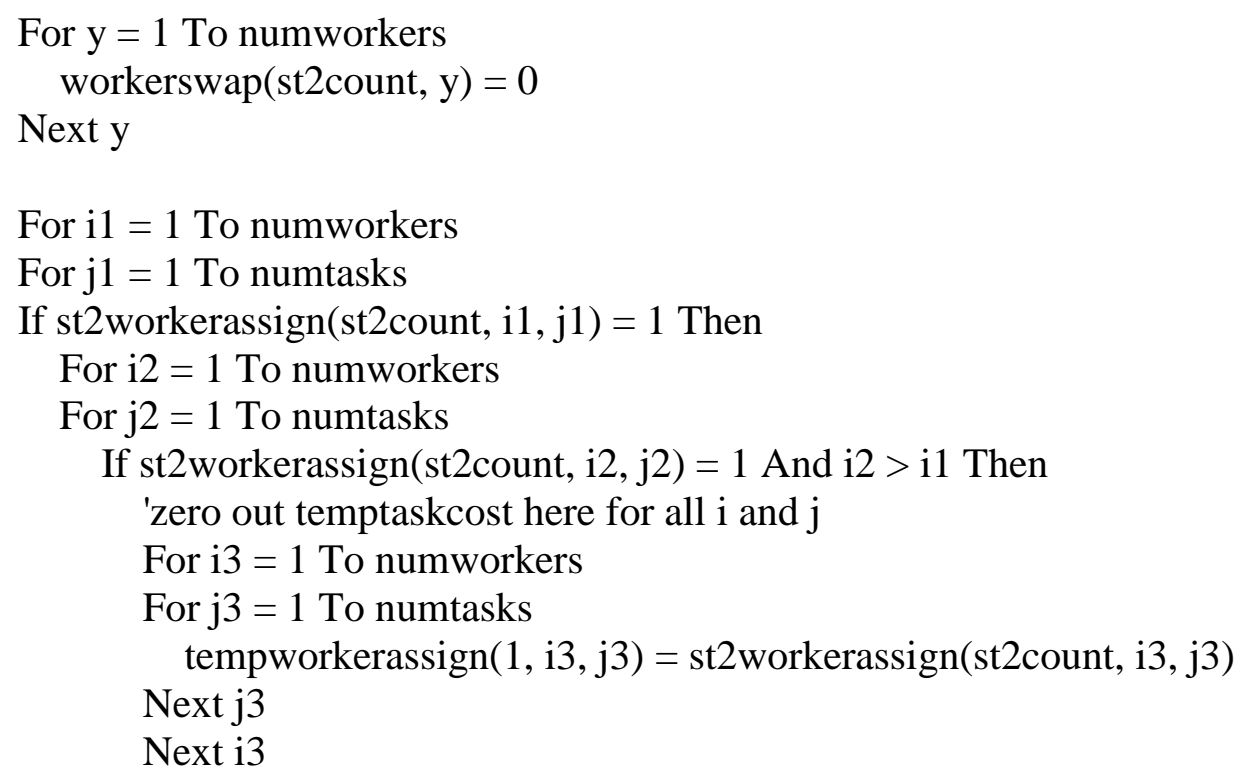

tempworkerassign $(1, \mathrm{i} 1, \mathrm{j} 1)=0$

tempworkerassign $(1, \mathrm{i} 2, \mathrm{j} 2)=0$

tempworkerassign $(1, \mathrm{i} 1, \mathrm{j} 2)=1$

tempworkerassign $(1, \mathrm{i} 2, \mathrm{j} 1)=1$

'we now have the new assignment to be considered against the Value Fn 


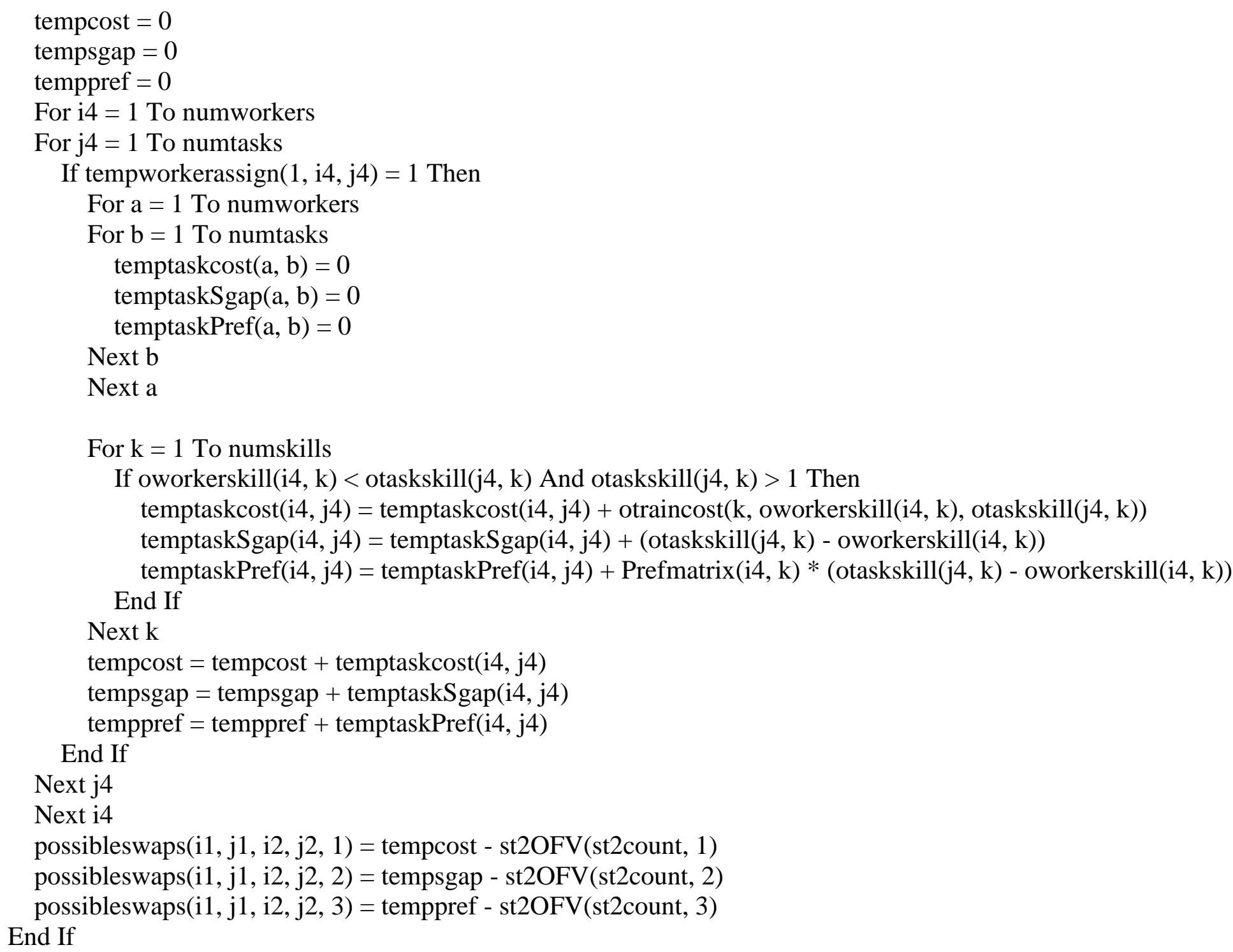




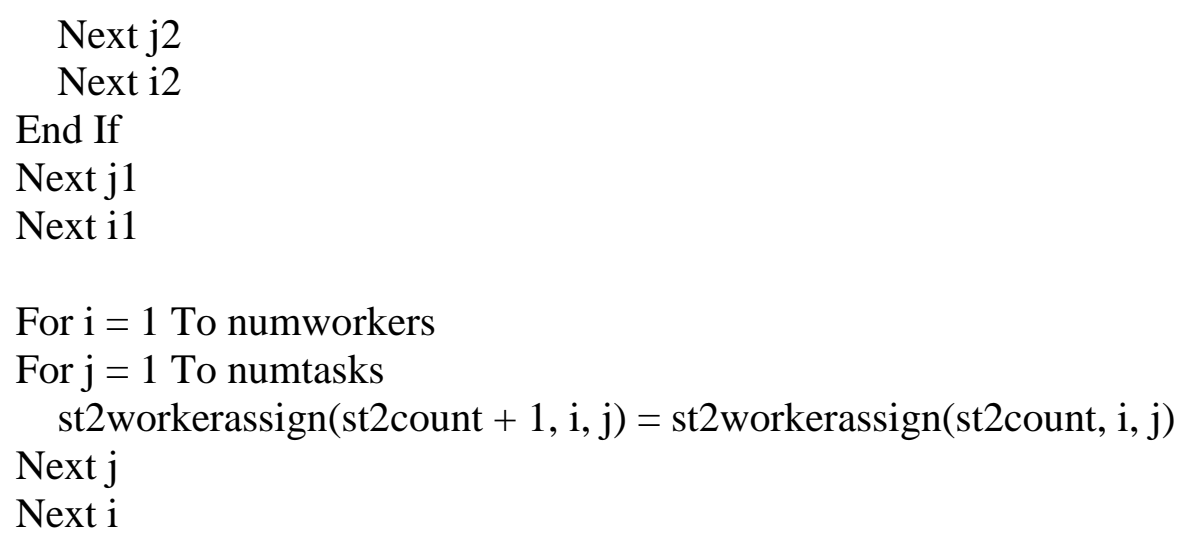

If baseOF( st 2 count $)=1$ Then

Do Until NEWsgap <=st2OFV(st2count, 2) * (1 - varB(2, st2count)) Or NEWpref <=st2OFV(st2count, 3)*(1 - varB(3, st2count)) mindeltacost $=0$

For $\mathrm{a}=1$ To numworkers

If workerswap(st2count, a) $=0$ Then

For $\mathrm{b}=1$ To numtasks

If st 2 workerassign( $(s t 2$ count, $a, b)=1$ Then

For $\mathrm{i}=1$ To numworkers

If workerswap(st2count, i) $=0$ Then

For $\mathrm{j}=1$ To numtasks

If st 2 workerassign(st 2 count, $i, j$ ) $=1$ Then

If possibleswaps $(\mathrm{a}, \mathrm{b}, \mathrm{i}, \mathrm{j}, \mathrm{1})<$ mindeltacost Then besti $1=\mathrm{a}$ 


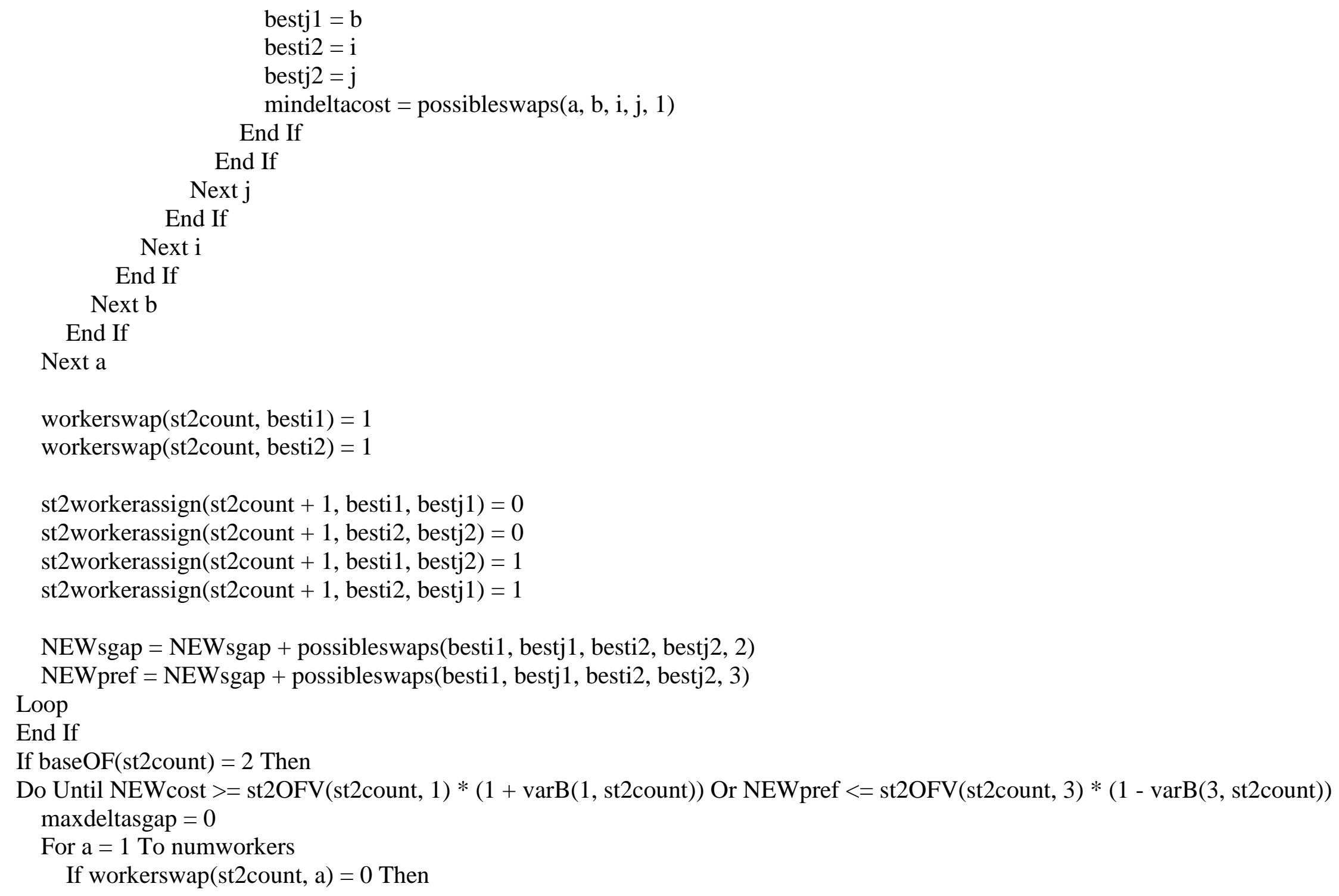




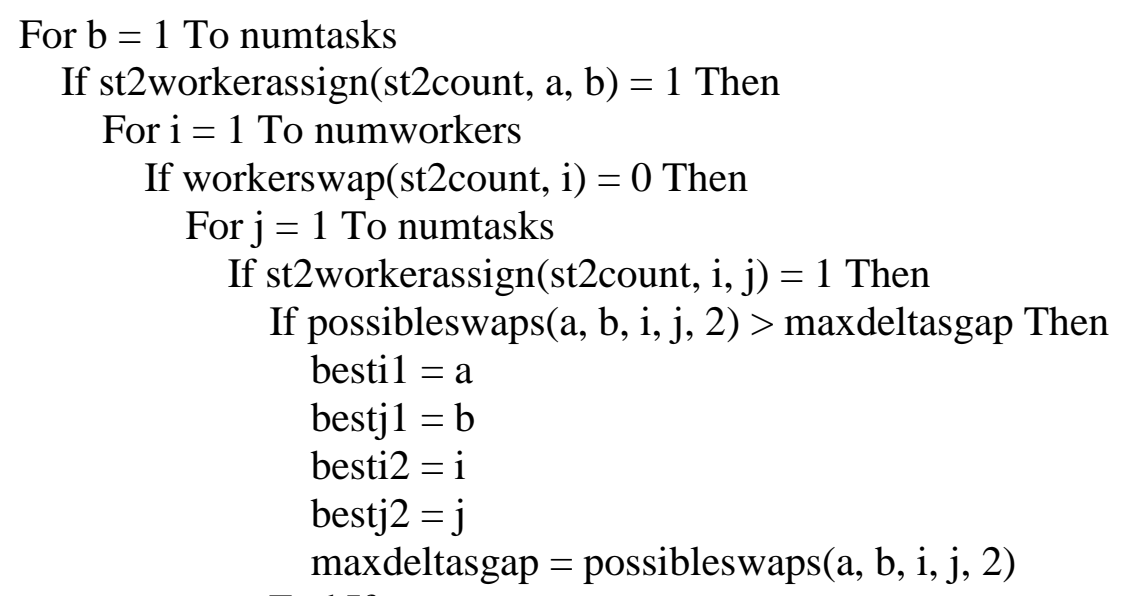

\section{End If}

\section{End If}

Next $j$

\section{End If}

Next i

\section{End If}

Next $b$

\section{End If}

Next a

workerswap $($ st 2 count, besti1 $)=1$

workerswap $($ st 2 count, besti2 $)=1$

st 2 workerassign $(\operatorname{st} 2$ count +1 , besti 1 , bestj 1$)=0$

st 2 workerassign (st 2 count +1 , besti 2 , bestj 2$)=0$

st 2 workerassign $($ st 2 count +1 , besti 1 , bestj 2$)=1$

st 2 workerassign $($ st 2 count +1 , besti 2 , best 11$)=1$

NEWcost $=$ NEWcost + possibleswaps $($ besti1, bestj1, besti2, bestj2, 1) 


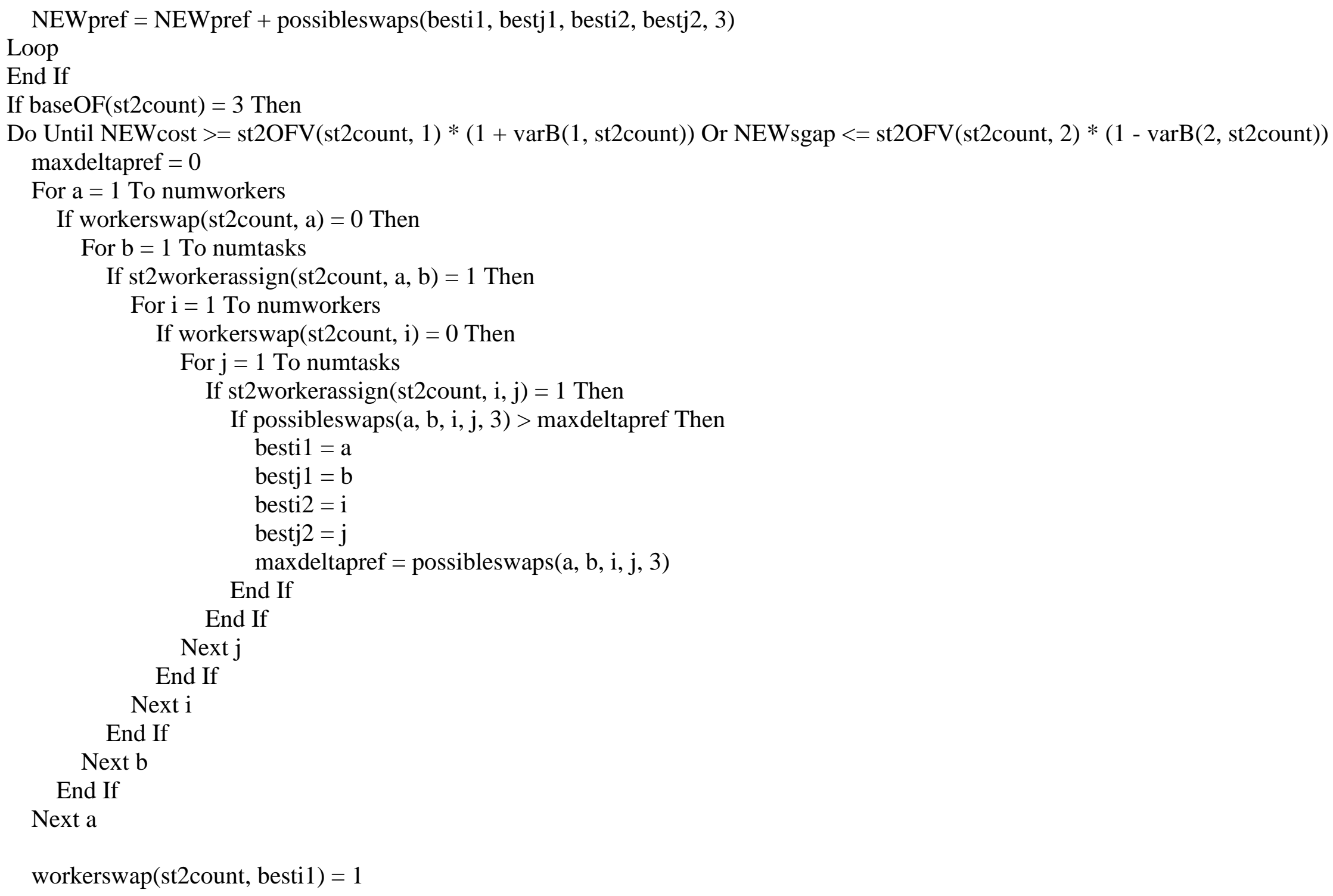


workerswap $($ st 2 count, besti 2$)=1$

st 2 workerassign $($ st 2 count +1 , besti 1 , bestj 1$)=0$

st 2 workerassign $($ st 2 count +1 , besti2, bestj 2$)=0$

st 2 workerassign $($ st 2 count +1 , besti 1 , bestj 2$)=1$

st 2 workerassign $($ st 2 count +1 , besti 2 , bestj 1$)=1$

NEWcost $=$ NEWcost + possibleswaps $($ besti1, bestj1, besti2, bestj2, 1)

Loop

NEWsgap = NEWsgap + possibleswaps $($ besti1, bestj1, besti2, bestj2, 2)

End If

st 2 count $=$ st 2 count +1

st2OFV $($ st 2 count, 1$)=0$

st $2 \mathrm{OFV}($ st 2 count, 2$)=0$

st $2 \mathrm{OFV}($ st 2 count, 3$)=0$

For i4 = 1 To numworkers

For j4 = 1 To numtasks

If st 2 workerassign(st2count, $\mathrm{i} 4, \mathrm{j} 4)=1$ Then

For $\mathrm{a}=1$ To numworkers

For $\mathrm{b}=1$ To numtasks

temptaskcost $(a, b)=0$

temptaskSgap $(\mathrm{a}, \mathrm{b})=0$

temptaskPref $(a, b)=0$

Next b

Next a

For $\mathrm{k}=1$ To numskills

If oworkerskill(i4, k) < otaskskill $(\mathrm{j} 4, \mathrm{k})$ And otaskskill( $(\mathrm{j} 4, \mathrm{k})>1$ Then 
temptaskcost $(\mathrm{i} 4, \mathrm{j} 4)=$ temptaskcost $(\mathrm{i} 4, \mathrm{j} 4)+\operatorname{otraincost}(\mathrm{k}$, oworkerskill $(\mathrm{i} 4, \mathrm{k}), \operatorname{otaskskill}(\mathrm{j} 4, \mathrm{k}))$

temptaskSgap $(\mathrm{i} 4, \mathrm{j} 4)=\operatorname{temptaskSgap}(\mathrm{i} 4, \mathrm{j} 4)+(\operatorname{otaskskill}(\mathrm{j} 4, \mathrm{k})-$ oworkerskill $(\mathrm{i} 4, \mathrm{k}))$

temptaskPref(i4, j4) = temptaskPref(i4, j4) + Prefmatrix $(\mathrm{i} 4, \mathrm{k}) *(\operatorname{otaskskill}(\mathrm{j} 4, \mathrm{k})-\operatorname{oworkerskill}(\mathrm{i} 4, \mathrm{k}))$

End If

Next $k$

$\operatorname{st} 2 \mathrm{OFV}($ st 2 count, 1$)=\operatorname{st} 2 \mathrm{OFV}($ st 2 count, 1$)+$ temptaskcost $(\mathrm{i} 4, \mathrm{j} 4)$

st $2 \mathrm{OFV}($ st 2 count, 2$)=\operatorname{st} 2 \mathrm{OFV}($ st 2 count, 2$)+$ temptaskSgap $(\mathrm{i} 4, \mathrm{j} 4)$

st2OFV(st2count, 3$)=\operatorname{st} 2 \mathrm{OFV}($ st2count, 3$)+\operatorname{temptaskPref}(\mathrm{i} 4, \mathrm{j} 4)$

End If

Next j4

Next i4

ActiveSheet.Cells $(1,2 *$ st 2 count $)=$ st 2 count

cellrow $=2$

For $\mathrm{i}=1$ To numworkers

For $\mathrm{j}=1$ To numtasks

If st2workerassign( $\mathrm{st} 2$ count, $i, j)=1$ Then

ActiveSheet.Cells(cellrow, st2count $* 2-1)=\mathrm{i}$

ActiveSheet.Cells (cellrow, st2count $* 2$ ) $=\mathrm{j}$

cellrow $=$ cellrow +1

End If

Next $\mathrm{j}$

Next i

ActiveSheet.Cells $(2+$ numtasks, st 2 count $* 2)=\operatorname{st} 2 \mathrm{OFV}($ st 2 count, 1$)$

ActiveSheet.Cells $(3+$ numtasks, st 2 count $* 2)=\operatorname{st} 2 \mathrm{OFV}($ st 2 count, 2$)$

ActiveSheet.Cells $(4+$ numtasks, st 2 count $* 2)=\operatorname{st} 2 \mathrm{OFV}($ st 2 count, 3$)$

usersatisfied = Application.InputBox("Is this a satisfactory assignment (1)? Run compromise stage again(0)", "") 
If usersatisfied $=1$ Then

MsgBox ("Done")

End If

If usersatisfied $<>1$ Then

Do Until baseOF $($ st 2 count $)=1$ Or baseOF $($ st 2 count $)=2$ Or baseOF $($ st 2 count $)=3$

baseOF(st2count) = InputBox("Which objective do you want to improve? (1-Cost, 2-Training, 3-Preference)", "") Loop

UserForm1.Label1.Caption = "To what percent would you like to compromise each objective? "

$\operatorname{varB}(1$, st2count $)=0$

$\operatorname{varB}(2$, st 2 count $)=0$

$\operatorname{varB}(3$, st 2 count $)=0$

If baseOF(st2count) $=2$ Then

'UserForm1.Label1.Caption = UserForm1.Label1.Caption \& baseOF(st2count) \& " from " \& st2OFV(st2count, 2) \& " to " \& st2OFV(st2count, 2)*1.1\&"(10\%)"

UserForm1.Frame1.Caption = "Total Cost (Obj 1)"

UserForm1.Frame2.Caption = "Worker Preference (Obj 3)"

UserForm1.OptionButton1.Caption $=$ st2OFV(st2count, 1) $* 0.04 \&$ \& (4\%)"

UserForm1.OptionButton2.Caption $=$ st2OFV(st2count, 1) * $0.07 \&$ \& (7\%)"

UserForm1.OptionButton3.Caption = st2OFV(st2count, 1) * $0.1 \&$ " (10\%)"

UserForm1.OptionButton4.Caption $=$ st2OFV(st2count, 1) * $0.13 \&$ " (13\%)"

UserForm 1.OptionButton5.Caption $=\operatorname{st2OFV}($ st 2 count, 1) $* 0.16 \&$ " $(16 \%) "$

UserForm1.OptionButton6.Caption = st2OFV(st2count, 3) * $0.04 \&$ \& (4\%)"

UserForm1.OptionButton7.Caption $=$ st2OFV (st2count, 3) * $0.07 \& "(7 \%) "$

UserForm1.OptionButton8.Caption = st2OFV(st2count, 3) * $0.1 \&$ " (10\%)"

UserForm1.OptionButton9.Caption $=\operatorname{st2OFV(st2count,~3)*0.13\& ~"~(13\% )"~}$

UserForm1.OptionButton10.Caption = st2OFV(st2count, 3) * $0.16 \&$ " (16\%)"

UserForm1.Show 
If $($ UserForm1.OptionButton1. Value $=$ True $)$ Then $\operatorname{varB}(1$, st 2 count $)=0.04$

If $($ UserForm1.OptionButton2.Value $=$ True $)$ Then $\operatorname{varB}(1$, st2count $)=0.07$

If $($ UserForm1.OptionButton3. Value $=$ True $)$ Then $\operatorname{varB}(1$, st2count $)=0.1$

If $($ UserForm1.OptionButton4. Value $=$ True $)$ Then $\operatorname{varB}(1$, st 2 count $)=0.13$

If $($ UserForm1.OptionButton5. Value $=$ True $)$ Then $\operatorname{varB}(1, \mathrm{st} 2$ count $)=0.16$

If $($ UserForm1.OptionButton6.Value $=$ True $)$ Then $\operatorname{varB}(3$, st 2 count $)=0.04$

If $($ UserForm1.OptionButton7. Value $=$ True $)$ Then $\operatorname{varB}(3$, st 2 count $)=0.07$

If $($ UserForm1.OptionButton8.Value $=$ True $)$ Then $\operatorname{varB}(3$, st 2 count $)=0.1$

If $($ UserForm1.OptionButton9. Value $=$ True $)$ Then $\operatorname{varB}(3$, st2count $)=0.13$

If $($ UserForm1.OptionButton10.Value $=$ True $)$ Then $\operatorname{varB}(3$, st 2 count $)=0.16$

End If

If baseOF(st 2 count $)=3$ Then

'UserForm1.Label1.Caption = UserForm1.Label1.Caption \& baseOF(st2count) \& " from " \& st2OFV(st2count, 3) \& " to " \& st2OFV(st2count, 3) * $1.1 \&$ "(10\%)"

UserForm1.Frame1.Caption $=$ "Total Cost $($ Obj 1)"

UserForm1.Frame2.Caption = "Training Amount (Obj 2)"

UserForm1.OptionButton1.Caption = st2OFV(st2count, 1) * $0.04 \&$ " (4\%)"

UserForm1.OptionButton2.Caption $=$ st2OFV(st2count, 1) * $0.07 \&$ " (7\%)"

UserForm 1.OptionButton3.Caption = st2OFV(st2count, 1) * $0.1 \&$ " (10\%)"

UserForm1.OptionButton4.Caption $=$ st2OFV(st2count, 1) * $0.13 \&$ \& (13\%)"

UserForm1.OptionButton5.Caption $=\operatorname{st2OFV}($ st2count, 1) $* 0.16 \& "(16 \%) "$

UserForm1.OptionButton1.Caption $=$ st2OFV(st2count, 2) $* 0.04 \& "(4 \%) "$

UserForm1.OptionButton2.Caption $=$ st2OFV(st2count, 2) * $0.07 \& "(7 \%) "$

UserForm1.OptionButton3.Caption $=\operatorname{st} 2 \mathrm{OFV}(\mathrm{st} 2 \mathrm{count}, 2) * 0.1 \& "(10 \%) "$

UserForm1.OptionButton4.Caption $=\operatorname{st} 2 \mathrm{OFV}($ st2 count, 2$) * 0.13 \& "(13 \%) "$

UserForm1.OptionButton5.Caption = st2OFV (st2count, 2) * $0.16 \&$ " (16\%)"

UserForm1.Show

If $($ UserForm1.OptionButton1. Value $=$ True $)$ Then $\operatorname{varB}(1$, st 2 count $)=0.04$

If $($ UserForm1.OptionButton2.Value $=$ True $)$ Then $\operatorname{varB}(1$, st 2 count $)=0.07$

If $($ UserForm1.OptionButton3. Value $=$ True $)$ Then $\operatorname{varB}(1$, st 2 count $)=0.1$ 
If $($ UserForm1.OptionButton4. Value $=$ True $)$ Then $\operatorname{varB}(1$, st 2 count $)=0.13$

If $($ UserForm1.OptionButton5. Value $=$ True $)$ Then $\operatorname{varB}(1$, st 2 count $)=0.16$

If $($ UserForm1.OptionButton1.Value $=$ True $)$ Then $\operatorname{varB}(2$, st 2 count $)=0.04$

If $($ UserForm1.OptionButton2. Value $=$ True $)$ Then $\operatorname{varB}(2$, st 2 count $)=0.07$

If $($ UserForm1.OptionButton3. Value $=$ True $)$ Then $\operatorname{varB}(2$, st 2 count $)=0.1$

If $($ UserForm1.OptionButton4.Value $=$ True $)$ Then $\operatorname{varB}(2$, st 2 count $)=0.13$

If $($ UserForm1.OptionButton5.Value $=$ True $)$ Then $\operatorname{varB}(2$, st 2 count $)=0.16$

End If

If baseOF(st2count $)=1$ Then

'UserForm1.Label1.Caption = UserForm1.Label1.Caption \& baseOF(st2count) \& " from " \& st2OFV(st2count, 1) \& " to " \& st2OFV(st2count, 1)*0.9\& "(10\%)"

UserForm1.Frame1.Caption = "Training Amount (Obj 2)"

UserForm1.Frame2.Caption = "Worker Preference $(\mathrm{Obj} 3) "$

UserForm1.OptionButton1.Caption = st2OFV(st2count, 2) * $0.04 \&$ " (4\%)"

UserForm 1.OptionButton2.Caption $=$ st2OFV(st2count, 2) $* 0.07 \&$ " (7\%)"

UserForm1.OptionButton3.Caption = st2OFV(st2count, 2) * $0.1 \&$ " (10\%)"

UserForm1.OptionButton4.Caption $=\operatorname{st} 2 \mathrm{OFV}($ st 2 count, 2$) * 0.13 \&$ " (13\%)"

UserForm1.OptionButton5.Caption $=\operatorname{st} 2 \mathrm{OFV}($ st2count, 2) $* 0.16 \& "(16 \%) "$

UserForm1.OptionButton6.Caption = st2OFV(st2count, 3) * $0.04 \&$ " (4\%)"

UserForm1.OptionButton7.Caption $=\operatorname{st2OFV(st2count,~3)*0.07~\& ~"~(7\% )"~}$

UserForm1.OptionButton8.Caption $=\operatorname{st2OFV(st2count,3)*0.1\& ~"(10\% )"~}$

UserForm1.OptionButton9.Caption = st2OFV(st2count, 3) * $0.13 \& "(13 \%) "$

UserForm1.OptionButton10.Caption = st2OFV(st2count, 3) * $0.16 \&$ " (16\%)"

UserForm1.Show

If $($ UserForm1.OptionButton1.Value $=$ True $)$ Then $\operatorname{varB}(2$, st 2 count $)=0.04$

If $($ UserForm1.OptionButton2.Value $=$ True $)$ Then $\operatorname{varB}(2$, st 2 count $)=0.07$

If $($ UserForm1.OptionButton3. Value $=$ True $)$ Then $\operatorname{varB}(2$, st 2 count $)=0.1$

If $($ UserForm1.OptionButton4. Value $=$ True $)$ Then $\operatorname{varB}(2$, st 2 count $)=0.13$

If $($ UserForm1.OptionButton5.Value $=$ True $)$ Then $\operatorname{varB}(2$, st 2 count $)=0.16$

If $($ UserForm1.OptionButton6.Value $=$ True $)$ Then $\operatorname{varB}(3$, st 2 count $)=0.04$ 
If $($ UserForm1.OptionButton7.Value $=$ True $)$ Then $\operatorname{varB}(3$, st 2 count $)=0.07$ If $($ UserForm1.OptionButton8.Value $=$ True $)$ Then $\operatorname{varB}(3$, st2count $)=0.1$

If $($ UserForm1.OptionButton9.Value $=$ True $)$ Then $\operatorname{varB}(3$, st 2 count $)=0.13$

If $($ UserForm1.OptionButton10.Value $=$ True $)$ Then $\operatorname{varB}(3$, st 2 count $)=0.16$

\section{End If}

\section{End If}

Loop 'this loop ends when usersatisfied=1

\section{End Sub}

